\title{
Anticoagulation for the long-term treatment of venous thromboembolism in people with cancer (Review)
}

Kahale LA, Hakoum MB, Tsolakian IG, Alturki F, Matar CF, Terrenato I, Sperati F, Barba M, Yosuico VED, Schünemann $\mathrm{H}$, Akl EA

Kahale LA, Hakoum MB, Tsolakian IG, Alturki F, Matar CF, Terrenato I, Sperati F, Barba M, Yosuico VED, Schünemann H, Akl EA. Anticoagulation for the long-term treatment of venous thromboembolism in people with cancer.

Cochrane Database of Systematic Reviews 2018, Issue 6. Art. No.: CD006650.

DOI: 10.1002/14651858.CD006650.pub5.

www.cochranelibrary.com 
TABLE OF CONTENTS

PLAIN LANGUAGE SUMMARY

SUMMARY OF FINDINGS

BACKGROUND

OBJECTIVES

METHODS

RESULTS

Figure 1.

Figure 2.

Figure 3.

DISCUSSION

AUTHORS' CONCLUSIONS

ACKNOWLEDGEMENTS

REFERENCES

CHARACTERISTICS OF STUDIES

DATA AND ANALYSES

Analysis 1.1. Comparison 1: Low molecular weight heparins (LMWH) versus vitamin K antagonists (VKA), Outcome 1: All-cause mortality (up to 6 months) (main analysis - active cancer)

Analysis 1.2. Comparison 1: Low molecular weight heparins (LMWH) versus vitamin K antagonists (VKA), Outcome 2: All-cause mortality (time-to-event)

Analysis 1.3. Comparison 1: Low molecular weight heparins (LMWH) versus vitamin K antagonists (VKA), Outcome 3: Recurrent venous thromboembolism (up to 6 months) (main analysis - active cancer)

Analysis 1.4. Comparison 1: Low molecular weight heparins (LMWH) versus vitamin K antagonists (VKA), Outcome 4: Recurrent venous thromboembolism (time-to-event)

Analysis 1.5. Comparison 1: Low molecular weight heparins (LMWH) versus vitamin K antagonists (VKA), Outcome 5: Major bleeding (up to 6 months) (main analysis - active cancer)

Analysis 1.6. Comparison 1: Low molecular weight heparins (LMWH) versus vitamin $\mathrm{K}$ antagonists (VKA), Outcome 6: Minor bleeding (up to 6 months) (main analysis - active cancer)

Analysis 1.7. Comparison 1: Low molecular weight heparins (LMWH) versus vitamin $\mathrm{K}$ antagonists (VKA), Outcome 7 : Thrombocytopenia (up to 6 months) (main analysis- active cancer)

Analysis 2.1. Comparison 2: Direct oral anticoagulants (DOAC) versus vitamin $\mathrm{K}$ antagonists (VKA), Outcome 1: All-cause mortality (6-12 months)

Analysis 2.2. Comparison 2: Direct oral anticoagulants (DOAC) versus vitamin K antagonists (VKA), Outcome 2: Recurrent venous thromboembolism (6-12 months)

Analysis 2.3. Comparison 2: Direct oral anticoagulants (DOAC) versus vitamin K antagonists (VKA), Outcome 3: Major bleeding (6-12 months)

Analysis 2.4. Comparison 2: Direct oral anticoagulants (DOAC) versus vitamin K antagonists (VKA), Outcome 4: Minor bleeding (6-12 months)

Analysis 3.1. Comparison 3: Direct oral anticoagulants (DOAC) versus low molecular weight heparins (LMWH), Outcome 1: Allcause mortality (6 months)

Analysis 3.2. Comparison 3: Direct oral anticoagulants (DOAC) versus low molecular weight heparins (LMWH), Outcome 2: Recurrent VTE (6 months)

Analysis 3.3. Comparison 3: Direct oral anticoagulants (DOAC) versus low molecular weight heparins (LMWH), Outcome 3: Major bleeding (6 months)

Analysis 3.4. Comparison 3: Direct oral anticoagulants (DOAC) versus low molecular weight heparins (LMWH), Outcome 4: Major GI bleeding (6 months)

Analysis 3.5. Comparison 3: Direct oral anticoagulants (DOAC) versus low molecular weight heparins (LMWH), Outcome 5: Major upper $\mathrm{Gl}$ bleeding (6 months)

Analysis 3.6. Comparison 3: Direct oral anticoagulants (DOAC) versus low molecular weight heparins (LMWH), Outcome 6: Major lower GI bleeding (6 months)

Analysis 3.7. Comparison 3: Direct oral anticoagulants (DOAC) versus low molecular weight heparins (LMWH), Outcome 7: Major non-GI bleeding (6 months)

Analysis 3.8. Comparison 3: Direct oral anticoagulants (DOAC) versus low molecular weight heparins (LMWH), Outcome 8: Minor bleeding (6 months) 
Analysis 3.9. Comparison 3: Direct oral anticoagulants (DOAC) versus low molecular weight heparins (LMWH), Outcome 9: Minor GI bleeding ( 6 months)

Analysis 3.10. Comparison 3: Direct oral anticoagulants (DOAC) versus low molecular weight heparins (LMWH), Outcome 10: Minor upper $\mathrm{GI}$ bleeding (6 months)

Analysis 3.11. Comparison 3: Direct oral anticoagulants (DOAC) versus low molecular weight heparins (LMWH), Outcome 11: Minor lower $\mathrm{GI}$ bleeding ( 6 months)

Analysis 3.12. Comparison 3: Direct oral anticoagulants (DOAC) versus low molecular weight heparins (LMWH), Outcome 12: Minor non-GI bleeding (6 months)

Analysis 4.1. Comparison 4: Idraparinux versus vitamin K antagonists (VKA), Outcome 1: All-cause mortality (up to 6 months) .. Analysis 4.2. Comparison 4: Idraparinux versus vitamin K antagonists (VKA), Outcome 2: Recurrent VTE (up to 6 months) ....... Analysis 4.3. Comparison 4: Idraparinux versus vitamin K antagonists (VKA), Outcome 3: Major bleeding (up to 6 months) ...... Analysis 4.4. Comparison 4: Idraparinux versus vitamin $\mathrm{K}$ antagonists (VKA), Outcome 4: Minor bleeding (up to 6 months) ...... ADDITIONAL TABLES

APPENDICES

WHAT'S NEW

HISTORY

CONTRIBUTIONS OF AUTHORS

DECLARATIONS OF INTEREST 
[Intervention Review]

\title{
Anticoagulation for the long-term treatment of venous thromboembolism in people with cancer
}

\author{
Lara A Kahale1, Maram B Hakoum², Ibrahim G Tsolakian¹, Fadel Alturki ${ }^{1}$, Charbel F Matar ${ }^{3}$, Irene Terrenato ${ }^{4}$, Francesca Sperati ${ }^{5}$, \\ Maddalena Barba 6 , Victor ED Yosuico ${ }^{7}$, Holger Schünemann ${ }^{8}$, Elie A Akl ${ }^{3}$ \\ 1Faculty of Medicine, American University of Beirut, Beirut, Lebanon. ${ }^{2}$ Family Medicine, American University of Beirut, Beirut, Lebanon. \\ ${ }^{3}$ Department of Internal Medicine, American University of Beirut Medical Center, Beirut, Lebanon. ${ }^{4}$ Biostatistics-Scientific Direction, \\ IRCCS Regina Elena National Cancer Institute, Rome, Italy. ${ }^{5}$ Biostatistics-Scientific Direction, Regina Elena National Cancer Institute, \\ Rome, Italy. ${ }^{6}$ Division of Medical Oncology 2 - Scientific Direction, IRCCS Regina Elena National Cancer Institute, Rome, Italy. ${ }^{7}$ Buffalo \\ Medical Group, Buffalo, New York, USA. ${ }^{8}$ Departments of Health Research Methods, Evidence, and Impact and of Medicine, McMaster \\ University, Hamilton, Canada
}

Contact: Elie A Akl, ea32@aub.edu.lb.

Editorial group: Cochrane Gynaecological, Neuro-oncology and Orphan Cancer Group.

Publication status and date: Edited (no change to conclusions), published in Issue 1, 2023.

Citation: Kahale LA, Hakoum MB, Tsolakian IG, Alturki F, Matar CF, Terrenato I, Sperati F, Barba M, Yosuico VED, Schünemann H, Akl EA. Anticoagulation for the long-term treatment of venous thromboembolism in people with cancer. Cochrane Database of Systematic Reviews 2018, Issue 6. Art. No.: CD006650. DOI: 10.1002/14651858.CD006650.pub5.

Copyright @ 2023 The Cochrane Collaboration. Published by John Wiley \& Sons, Ltd.

\section{A B S T R A C T}

\section{Background}

Cancer increases the risk of thromboembolic events, especially in people receiving anticoagulation treatments.

\section{Objectives}

To compare the efficacy and safety of low molecular weight heparins (LMWHs), direct oral anticoagulants (DOACs), vitamin K antagonists (VKAs), and other anticoagulants for the long-term treatment of venous thromboembolism (VTE) in people with cancer.

\section{Search methods}

We conducted a literature search including a comprehensive electronic search of the Cochrane Central Register of Controlled Trials ), MEDLINE (Ovid), and Embase (Ovid); handsearching conference proceedings; checking references of included studies; and a search for ongoing studies in trial registries. As part of the living systematic review approach, we run searches continually, incorporating new evidence after it is identified. Last search date 14 May 2021.

\section{Selection criteria}

Randomized controlled trials (RCTs) assessing the benefits and harms of long-term treatment with LMWHs, DOACs, VKAs, or other anticoagulants in people with cancer and symptomatic VTE.

\section{Data collection and analysis}

We extracted data in duplicate on study characteristics and risk of bias. Outcomes included: all-cause mortality, recurrent VTE, major bleeding, minor bleeding, thrombocytopenia, and health-related quality of life (QoL). We assessed the certainty of the evidence at the outcome level following the GRADE approach (GRADE handbook [GRADE handbook]).

\section{Main results}

Of 3583 citations, 19 RCTs fulfilled the eligibility criteria. 


\section{Low molecular weight heparins versus vitamin $\mathrm{K}$ antagonists}

Eight studies enrolling 2327 participants compared LMWHs with VKAs. Meta-analysis of five studies probably did not rule out a beneficial or harmful effect of LMWHs compared to VKAs on mortality up to 12 months of follow-up (risk ratio (RR) 1.00, 95\% confidence interval (CI) 0.88 to 1.13; risk difference (RD) 0 fewer per $1000,95 \% \mathrm{Cl} 45$ fewer to 48 more; moderate-certainty evidence). Meta-analysis of four studies did not rule out a beneficial or harmful effect of LMWHs compared to VKAs on major bleeding (RR 1.09, 95\% $\mathrm{Cl} 0.55$ to 2.12 ; RD 4 more per $1000,95 \% \mathrm{Cl} 19$ fewer to 48 more, moderate-certainty evidence) or minor bleeding (RR $0.78,95 \% \mathrm{Cl} 0.47$ to 1.27 ; RD 38 fewer per $1000,95 \%$ $\mathrm{Cl} 92$ fewer to 47 more; low-certainty evidence), or thrombocytopenia (RR $0.94,95 \% \mathrm{Cl} 0.52$ to 1.69). Meta-analysis of five studies showed that LMWHs probably reduced the recurrence of VTE compared to VKAs (RR $0.58,95 \% \mathrm{Cl} 0.43$ to 0.77 ; RD 53 fewer per $1000,95 \% \mathrm{Cl} 29 \mathrm{fewer}$ to 72 fewer, moderate-certainty evidence).

\section{Direct oral anticoagulants versus vitamin $\mathrm{K}$ antagonists}

Five studies enrolling 982 participants compared DOACs with VKAs. Meta-analysis of four studies may not rule out a beneficial or harmful effect of DOACs compared to VKAs on mortality (RR 0.93, 95\% CI 0.71 to 1.21; RD 12 fewer per 1000, 95\% CI 51 fewer to 37 more; low-certainty evidence), recurrent VTE (RR 0.66, 95\% Cl 0.33 to 1.31; RD 14 fewer per 1000, 95\% Cl 27 fewer to 12 more; low-certainty evidence), major bleeding (RR $0.77,95 \% \mathrm{Cl} 0.38$ to 1.57 , RD 8 fewer per $1000,95 \% \mathrm{Cl} 22$ fewer to 20 more; low-certainty evidence), or minor bleeding (RR $0.84,95 \% \mathrm{Cl} 0.58$ to 1.22 ; RD 21 fewer per $1000,95 \% \mathrm{Cl} 54$ fewer to 28 more; low-certainty evidence). One study reporting on DOAC versus VKA was published as abstract so is not included in the main analysis.

\section{Direct oral anticoagulants versus low molecular weight heparins}

Two studies enrolling 1455 participants compared DOAC with LMWH. The study by Raskob did not rule out a beneficial or harmful effect of DOACs compared to LMWH on mortality up to 12 months of follow-up (RR 1.07, 95\% Cl 0.92 to 1.25; RD 27 more per 1000, 95\% Cl 30 fewer to 95 more; low-certainty evidence). The data also showed that DOACs may have shown a likely reduction in VTE recurrence up to 12 months of follow-up compared to LMWH (RR $0.69,95 \% \mathrm{Cl} 0.47$ to 1.01 ; RD 36 fewer per $1000,95 \% \mathrm{Cl} 62$ fewer to 1 more; low-certainty evidence). DOAC may have increased major bleeding at 12 months of follow-up compared to LMWH (RR 1.71,95\% CI 1.01 to 2.88; RD 29 more per 1000 , $95 \% \mathrm{Cl} 0$ fewer to 78 more; low-certainty evidence) and likely increased minor bleeding up to 12 months of follow-up compared to LMWH (RR 1.31, 95\% Cl 0.95 to 1.80; RD 35 more per 1000, 95\% Cl 6 fewer to 92 more; low-certainty evidence). The second study on DOAC versus LMWH was published as an abstract and is not included in the main analysis.

\section{Idraparinux versus vitamin $\mathrm{K}$ antagonists}

One RCT with 284 participants compared once-weekly subcutaneous injection of idraparinux versus standard treatment (parenteral anticoagulation followed by warfarin or acenocoumarol) for three or six months. The data probably did not rule out a beneficial or harmful effect of idraparinux compared to VKAs on mortality at six months (RR 1.11, 95\% Cl 0.78 to 1.59; RD 31 more per 1000, 95\% Cl 62 fewer to 167 more; moderate-certainty evidence), VTE recurrence at six months (RR 0.46, 95\% Cl 0.16 to 1.32 ; RD 42 fewer per $1000,95 \% \mathrm{Cl} 65$ fewer to 25 more; low-certainty evidence) or major bleeding (RR 1.11, 95\% Cl 0.35 to 3.56; RD 4 more per $1000,95 \% \mathrm{Cl} 25$ fewer to 98 more; low-certainty evidence).

\section{Authors' conclusions}

For the long-term treatment of VTE in people with cancer, evidence shows that LMWHs compared to VKAs probably produces an important reduction in VTE and DOACs compared to LMWH, may likely reduce VTE but may increase risk of major bleeding. Decisions for a person with cancer and VTE to start long-term LMWHs versus oral anticoagulation should balance benefits and harms and integrate the person's values and preferences for the important outcomes and alternative management strategies.

Editorial note: this is a living systematic review (LSR). LSRs offer new approaches to review updating in which the review is continually updated, incorporating relevant new evidence as it becomes available. Please refer to the Cochrane Database of Systematic Reviews for the current status of this review.

\section{PLAIN LANGUAGE SUMMARY}

\section{Blood thinners for the long-term treatment of blood clots in people with cancer}

\section{Background}

People with cancer are at an increased risk of developing blood clots and might respond differently to different types of blood thinners (anticoagulants).

\section{Study characteristics}

We searched scientific databases for clinical trials looking at the effects of long-term treatment with different blood thinners on blood clot recurrence in people with cancer with a confirmed diagnosis of deep venous thrombosis (a blood clot in the limbs) or pulmonary embolism (a blood clot in the lungs). We included trials with any type of cancer, and irrespective of the type of cancer treatment. The trials looked at survival, recurrent blood clot, bleeding and blood platelet levels (which are involved in blood clotting). The evidence was current to May 2021.

\section{Key results}


We found 18trials enrollingparticipants with cancer and blood clots. The studies found that low molecular weight heparins (LMWHs; a type of blood thinner that is injected into a vein) were superior to vitamin $\mathrm{K}$ antagonists (VKAs; a type of blood thinner taken by mouth (oral)) in reducing the recurrence of blood clots. The available data did not provide a clear answer about the effects of these drugs on death and the side effect of bleeding. The studies also found that direct oral anticoagulants (DOACs; another type of blood thinner taken by mouth) might decrease the recurrence of blood clots compared to LMWH while increasing the risk of bleeding. There was no clear answer when comparing DOACs (a newer type of oral blood thinner) and VKAs (an older type of oral blood thinner) for death, blood clot recurrence and bleeding.

\section{Reliability of the evidence}

When comparing LMWHs to VKAs, we judged the certainty of the evidence to be moderate for recurrent blood clots, death at one year and major bleeding, and low for minor bleeding.

When comparing DOACs to VKAs, we judged the certainty of the evidence to be low for death, recurrent blood clots and bleeding complications.

Editorial note: this is a living systematic review. Living systematic reviews offer a new approach to review updating in which the review is continually updated, incorporating relevant new evidence as it becomes available. Please refer to the Cochrane Database of Systematic Reviews for the current status of this review. 


\section{S U M M ARY OF F I N D I N G}

Summary of findings 1 . Low molecular weight heparin secondary prophylaxis compared to vitamin $\mathrm{K}$ antagonist secondary prophylaxis in people with cancer with venous thromboembolism

Low molecular weight heparin secondary prophylaxis compared to vitamin $\mathrm{K}$ antagonist secondary prophylaxis in patients with cancer with venous thromboembolism

Population: People with cancer with venous thromboembolism

Setting: Outpatient

Intervention: LMWH secondary prophylaxis

Comparison: VKA secondary prophylaxis

\begin{tabular}{|c|c|c|c|c|c|}
\hline \multirow[t]{2}{*}{ Outcomes } & \multirow{2}{*}{$\begin{array}{l}\text { № of participants } \\
\text { (studies) } \\
\text { Follow up }\end{array}$} & \multirow{2}{*}{$\begin{array}{l}\text { Certainty of the } \\
\text { evidence } \\
\text { (GRADE) }\end{array}$} & \multirow{2}{*}{$\begin{array}{l}\text { Relative effect } \\
(95 \% \mathrm{CI})\end{array}$} & \multicolumn{2}{|c|}{ Anticipated absolute effects ${ }^{*}(95 \% \mathrm{Cl})$} \\
\hline & & & & $\begin{array}{l}\text { Risk with VKA sec- } \\
\text { ondary prophylax- } \\
\text { is }\end{array}$ & $\begin{array}{l}\text { Risk difference with LMWH sec- } \\
\text { ondary prophylaxis }\end{array}$ \\
\hline $\begin{array}{l}\text { All-cause mortality (main analysis - ac- } \\
\text { tive cancer) } \\
\text { follow up: } 6 \text { months }\end{array}$ & $\begin{array}{l}1712 \\
\text { (4 RCTs) }\end{array}$ & $\begin{array}{l}\oplus \oplus \Theta \Theta \\
\mathrm{LOW} 12\end{array}$ & $\begin{array}{l}\text { RR } 0.99 \\
(0.88 \text { to } 1.12)\end{array}$ & 374 per 1,000 & $\begin{array}{l}4 \text { fewer per } 1,000 \\
\text { ( } 45 \text { fewer to } 45 \text { more) }\end{array}$ \\
\hline \multirow[t]{2}{*}{ All-cause mortality (time-to-event) } & \multirow{2}{*}{$\begin{array}{l}1243 \\
(2 \text { RCTs) }\end{array}$} & \multirow{2}{*}{$\begin{array}{l}\oplus \oplus \odot \ominus \\
\text { LOW } 23\end{array}$} & \multirow{2}{*}{$\begin{array}{l}\text { HR } 0.94 \\
(0.74 \text { to } 1.20)\end{array}$} & Study population & \\
\hline & & & & 374 per 1,000 & $\begin{array}{l}18 \text { fewer per } 1,000 \\
\text { (81 fewer to } 56 \text { more) }\end{array}$ \\
\hline \multirow{2}{*}{$\begin{array}{l}\text { Recurrent venous thromboembolism } \\
\text { (main analysis - active cancer) } \\
\text { follow up: } 6 \text { months }\end{array}$} & \multirow{2}{*}{$\begin{array}{l}1712 \\
\text { (4 RCTs) }\end{array}$} & \multirow{2}{*}{$\begin{array}{l}\oplus \oplus \oplus \odot \\
\text { MODERATE } 1\end{array}$} & \multirow{2}{*}{$\begin{array}{l}\text { RR } 0.59 \\
(0.44 \text { to } 0.80)\end{array}$} & Study population & \\
\hline & & & & 124 per 1,000 & $\begin{array}{l}51 \text { fewer per } 1,000 \\
\text { (69 fewer to } 25 \text { fewer) }\end{array}$ \\
\hline \multirow{2}{*}{$\begin{array}{l}\text { Recurrent venous thromboembolism } \\
\text { (time-to-event) }\end{array}$} & \multirow{2}{*}{$\begin{array}{l}1243 \\
\text { (2 RCTs) }\end{array}$} & \multirow{2}{*}{$\begin{array}{l}\oplus \oplus \oplus \odot \\
\text { MODERATE }\end{array}$} & \multirow{2}{*}{$\begin{array}{l}\text { HR } 0.49 \\
\text { (0.31 to } 0.78)\end{array}$} & Study population & \\
\hline & & & & 124 per 1,000 & $\begin{array}{l}61 \text { fewer per } 1,000 \\
\text { ( } 84 \text { fewer to } 26 \text { fewer) }\end{array}$ \\
\hline \multirow{2}{*}{$\begin{array}{l}\text { Major bleeding (main analysis - active } \\
\text { cancer) } \\
\text { follow up: } 6 \text { months }\end{array}$} & \multirow[t]{2}{*}{$\begin{array}{l}1712 \\
\text { (4 RCTs) }\end{array}$} & \multirow[t]{2}{*}{$\begin{array}{l}\oplus \oplus \ominus \ominus \\
\text { LOW } 124\end{array}$} & \multirow[t]{2}{*}{$\begin{array}{l}\text { RR } 1.09 \\
\text { (0.55 to } 2.12 \text { ) }\end{array}$} & Study population & \\
\hline & & & & 43 per 1,000 & $\begin{array}{l}4 \text { more per } 1,000 \\
\text { (19 fewer to } 48 \text { more) }\end{array}$ \\
\hline
\end{tabular}









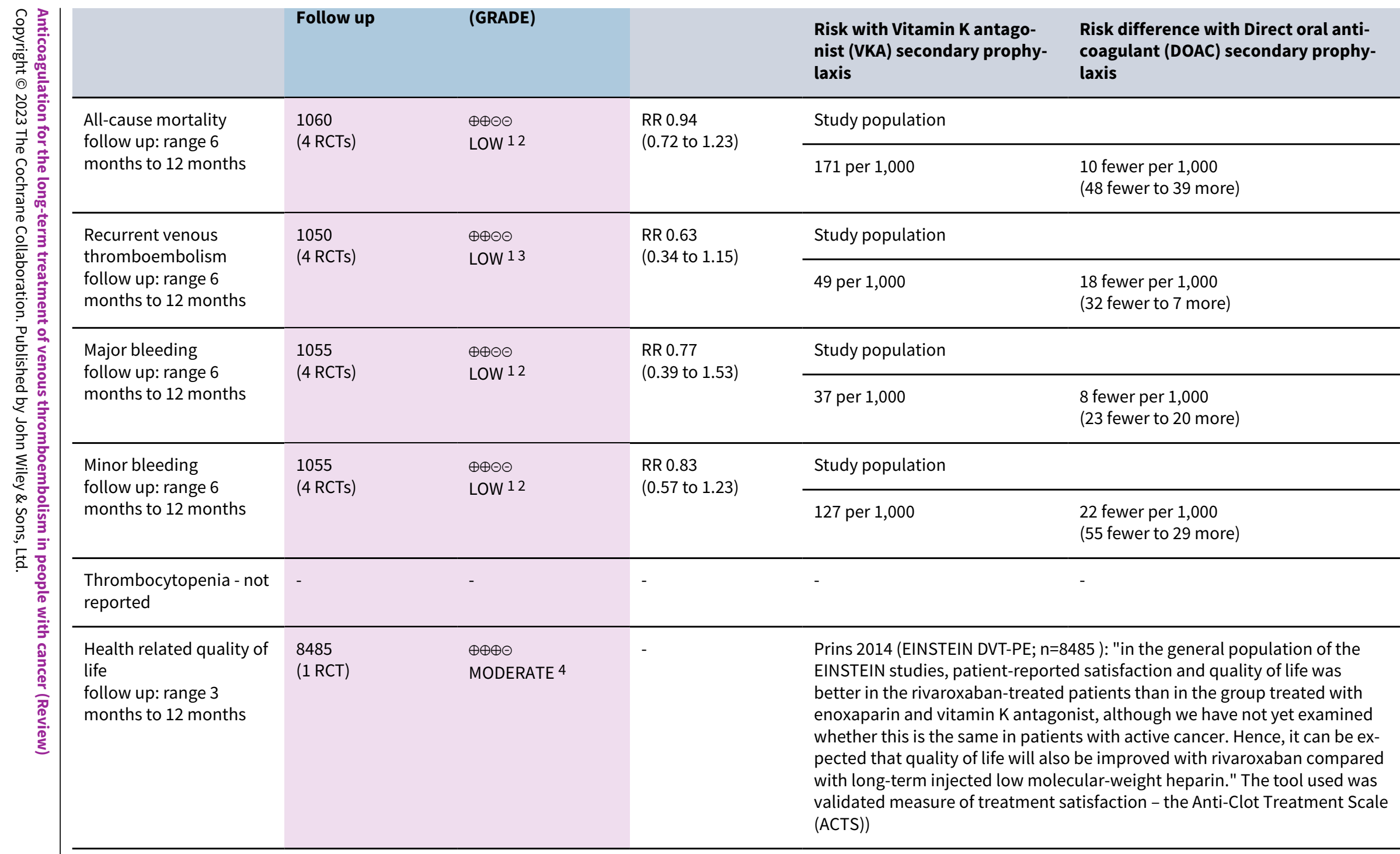

*The risk in the intervention group (and its 95\% confidence interval) is based on the assumed risk in the comparison group and the relative effect of the intervention (and its $95 \% \mathrm{Cl})$.

Cl: Confidence interval; RR: Risk ratio; OR: Odds ratio;

\section{GRADE Working Group grades of evidence}

High certainty: We are very confident that the true effect lies close to that of the estimate of the effect 
Moderate certainty: We are moderately confident in the effect estimate: The true effect is likely to be close to the estimate of the effect, but there is a possibility that it is substantially different

Low certainty: Our confidence in the effect estimate is limited: The true effect may be substantially different from the estimate of the effect

Very low certainty: We have very little confidence in the effect estimate: The true effect is likely to be substantially different from the estimate of effect

1 Some concern with indirectness (study by Schulman et al (RECOVER I-II) included patients with a diagnosis of cancer within five years before enrollment), however the weight of these studies was low and heterogeneity was very low.

2 Downgraded by two levels due to very serious imprecision. Confidence interval suggests both potential benefit and potential harm.

3 Downgraded by two levels due to very serious imprecision. Confidence interval suggests both potential benefit and potential no effect. Low number of events.

4 Downgraded by one level for serious indirectness. The study by Prins and colleagues (Prins 2014 ( EINSTEIN n=8485)) reports health related quality of life for the whole study population, without providing data for the cancer subgroup

\section{Summary of findings 4. Direct oral anticoagulant secondary prophylaxis compared to Low molecular weight heparin secondary prophylaxis in} patients with cancer with venous thromboembolism

Direct oral anticoagulant secondary prophylaxis compared to Low molecular weight heparin secondary prophylaxis in patients with cancer with venous thromboembolism

Patient or population: patients with cancer with venous thromboembolism

Setting: Outpatient

Intervention: DOAC secondary prophylaxis

Comparison: LMWH secondary prophylaxis

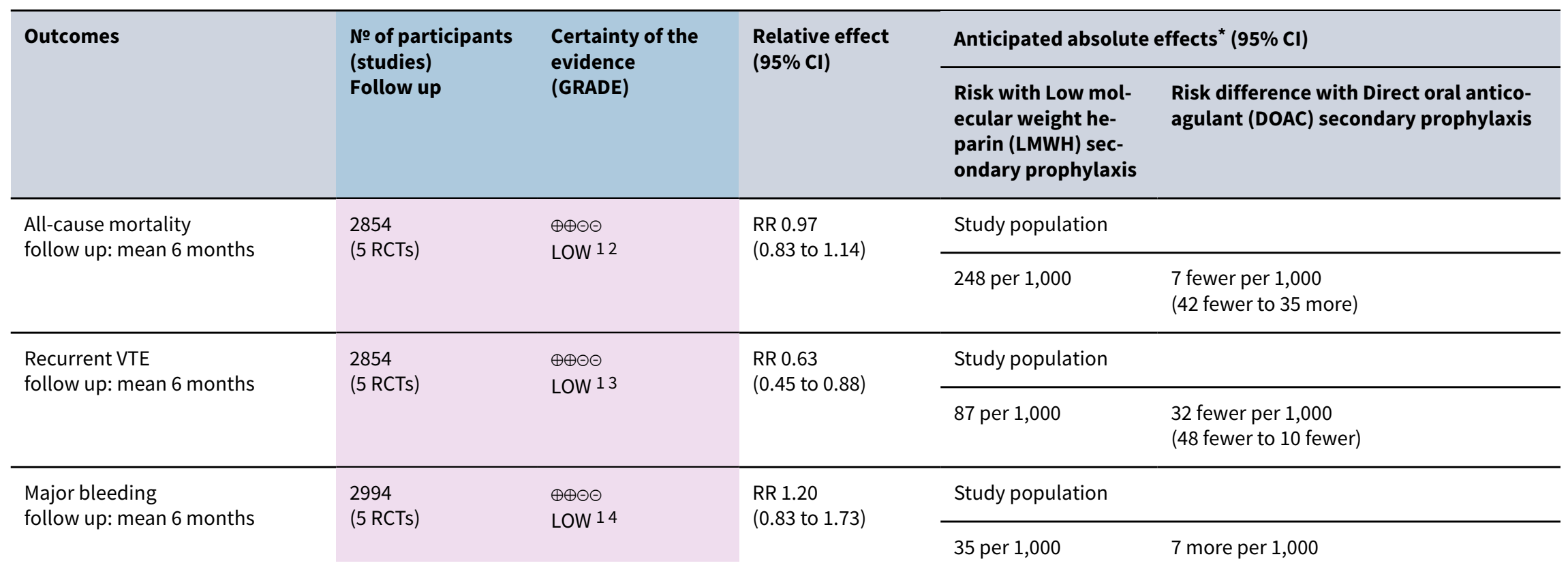




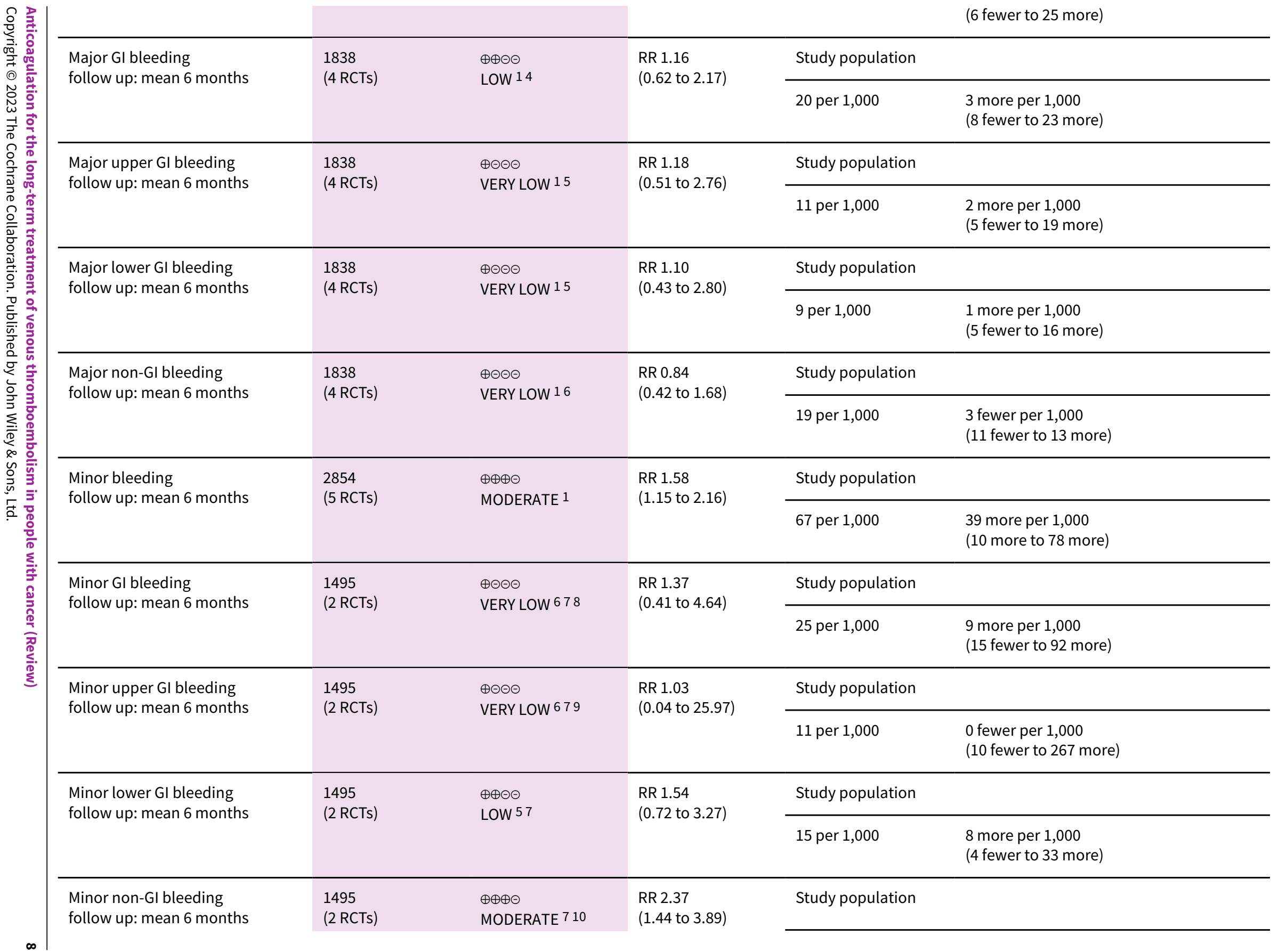


* The risk in the intervention group (and its 95\% confidence interval) is based on the assumed risk in the comparison group and the relative effect of the intervention (and its $95 \% \mathrm{Cl})$.

Cl: Confidence interval; RR: Risk ratio; OR: Odds ratio;

\section{GRADE Working Group grades of evidence}

High certainty: We are very confident that the true effect lies close to that of the estimate of the effect

Moderate certainty: We are moderately confident in the effect estimate: The true effect is likely to be close to the estimate of the effect, but there is a possibility that it is substantially different

Low certainty: Our confidence in the effect estimate is limited: The true effect may be substantially different from the estimate of the effect

Very low certainty: We have very little confidence in the effect estimate: The true effect is likely to be substantially different from the estimate of effect

1 Downgraded by one level due to serious risk of bias. Allocation concealment was not reported in one study, lack of blinding of patients and personnel in all studies, and high risk of bias related to incomplete outcome data.

2 Downgraded by one level due to serious imprecision. Confidence interval suggests both potential benefit and potential harm.

3 Downgraded by one level due to serious imprecision. Confidence interval suggests both potential benefit and potential no effect.

4 Downgraded by one level due to serious imprecision. Confidence interval suggests both potential harm and potential no effect.

5 Downgraded by two levels due to very serious imprecision. Confidence interval suggests both potential harm and potential no effect. Low number of events.

6 Downgraded by two levels due to very serious imprecision. Confidence interval suggests both potential harm and potential benefit. Low number of events.

7 Some concern with risk of bias. Lack of blinding of patients and personnel in both studies.

8 Downgraded by one level due to serious inconsistency (unexplained heterogeneity $12=64 \%$ ) and due to some concern with risk of bias.

9 Downgraded by two levels due to very serious inconsistency (unexplained heterogeneity $12=74 \%$.) and due to some concern with risk of bias.

10 Downgraded by one level due to serious imprecision. Low number of events.

Summary of findings 5. Idraparinux secondary prophylaxis compared to vitamin $\mathrm{K}$ antagonist secondary prophylaxis in people with cancer with venous thromboembolism

Idraparinux secondary prophylaxis compared to VKA secondary prophylaxis in people with cancer with VTE

Population: people with cancer with VTE receiving secondary prophylaxis

Setting: outpatient

Intervention: idraparinux prophylaxis

Control: VKA prophylaxis

\section{Outcomes}

\section{№ of participants}

(studies)

\section{Certainty of the}

evidence

(GRADE)

\section{Relative effect}

(95\% Cl)
Anticipated absolute effects ${ }^{\star}(95 \% \mathrm{Cl})$

$\bullet$ 


\begin{tabular}{|c|c|c|c|c|c|}
\hline & & & & $\begin{array}{l}\text { Risk with VKA sec- } \\
\text { ondary prophylaxis }\end{array}$ & $\begin{array}{l}\text { Risk difference with idraparinux sec- } \\
\text { ondary prophylaxis }\end{array}$ \\
\hline \multirow{2}{*}{$\begin{array}{l}\text { All-cause mortality } \\
\text { follow-up: mean } 6 \text { months }\end{array}$} & \multirow{2}{*}{$\begin{array}{l}284 \\
(1 \mathrm{RCT})\end{array}$} & \multirow{2}{*}{$\begin{array}{l}\oplus \oplus \oplus \odot \\
\text { Moderate } a\end{array}$} & \multirow{2}{*}{$\begin{array}{l}\text { RR } 1.11 \\
(0.78 \text { to } 1.59)\end{array}$} & \multicolumn{2}{|l|}{ Study population } \\
\hline & & & & 283 per 1000 & $\begin{array}{l}31 \text { more per } 1000 \\
\text { (62 fewer to } 167 \text { more) }\end{array}$ \\
\hline \multirow{2}{*}{$\begin{array}{l}\text { Recurrent VTE } \\
\text { follow-up: mean } 6 \text { months }\end{array}$} & \multirow{2}{*}{$\begin{array}{l}270 \\
(1 \mathrm{RCT})\end{array}$} & \multirow{2}{*}{$\begin{array}{l}\oplus \oplus \odot \odot \\
\mathbf{L o w}^{b}\end{array}$} & \multirow{2}{*}{$\begin{array}{l}\text { RR } 0.46 \\
(0.16 \text { to } 1.32)\end{array}$} & \multicolumn{2}{|l|}{ Study population } \\
\hline & & & & 77 per 1000 & $\begin{array}{l}42 \text { fewer per } 1000 \\
\text { ( } 65 \text { fewer to } 25 \text { more) }\end{array}$ \\
\hline \multirow{2}{*}{$\begin{array}{l}\text { Major bleeding } \\
\text { follow-up: mean } 6 \text { months }\end{array}$} & \multirow[t]{2}{*}{$\begin{array}{l}270 \\
(1 \mathrm{RCT})\end{array}$} & \multirow[t]{2}{*}{$\begin{array}{l}\oplus \oplus \oplus \ominus \\
\text { Low }^{c}\end{array}$} & \multirow[t]{2}{*}{$\begin{array}{l}\text { RR } 1.11 \\
\text { (0.35 to } 3.56)\end{array}$} & \multicolumn{2}{|l|}{ Study population } \\
\hline & & & & 38 per 1000 & $\begin{array}{l}4 \text { more per } 1000 \\
\text { ( } 25 \text { fewer to } 98 \text { more) }\end{array}$ \\
\hline Minor bleeding - not reported & - & - & - & - & - \\
\hline $\begin{array}{l}\text { Health-related quality of life - } \\
\text { not reported }\end{array}$ & - & - & - & - & - \\
\hline \multicolumn{6}{|c|}{$\begin{array}{l}\text { *The risk in the intervention group (and its } 95 \% \text { confidence interval) is based on the assumed risk in the comparison group and the relative effect of the intervention (and } \\
\text { its } 95 \% \mathrm{Cl} \text { ) }\end{array}$} \\
\hline
\end{tabular}

\section{GRADE Working Group grades of evidence}

High certainty: we are very confident that the true effect lies close to that of the estimate of the effect.

Moderate-certainty: we are moderately confident in the effect estimate: the true effect is likely to be close to the estimate of the effect, but there is a possibility that it is substantially different.

Low-certainty: our confidence in the effect estimate is limited: the true effect may be substantially different from the estimate of the effect.

Very low-certainty: we have very little confidence in the effect estimate: the true effect is likely to be substantially different from the estimate of effect.

${ }^{a}$ Downgraded one level due to serious imprecision, $95 \% \mathrm{Cl}$ was consistent with the possibility for important benefit (62 per 1000 absolute reduction) and possibility of important harm (167 per 1000 absolute increase), included 85 events.

$b$ Downgraded two level due to very serious imprecision, $95 \% \mathrm{Cl}$ was consistent with the possibility of important benefit ( 65 fewer per 1000$)$ and possibility of important harm (25 more per 1000); included 15 events.

cDowngraded two levels due to very serious imprecision, $95 \% \mathrm{Cl}$ was consistent with the possibility for important benefit ( 25 per 1000 absolute reduction) and possibility of important harm (98 per 1000 absolute increase), included 11 events. 


\section{B A C K G R O U N D}

Please refer to the glossary for the definitions of technical terms (Table 1).

\section{Description of the condition}

Cancer is associated with an increased risk of venous thromboembolism (VTE) of four- to six-fold (Heit 2000). Cancerrelated interventions such as chemotherapy, hormonal therapy and indwelling central venous catheters also increase the risk of VTE (Heit 2000). Similarly, people undergoing surgery for cancer have a higher risk of VTE than people undergoing surgery for diseases other than cancer (Gallus 1997; Kakkar 1970). Furthermore, people with cancer and VTE have a higher risk of death than people with cancer alone or with VTE alone (Levitan 1999; Sorensen 2000).

People with cancer also have different benefits and risks from anticoagulant treatment than people without cancer. For instance, during oral anticoagulation therapy for VTE, people with cancer, compared with people without cancer, have a higher incidence of recurrent VTE (27.1 events per 100 participant-years with cancer versus 9.0 events per 100 participant-years without cancer; $P=$ 0.003 ) and of major bleeding (13.3 events per 100 participant-years with cancer versus 2.2 events per 100 participant-years without cancer; $\mathrm{P}=0.002$ ) (Hutten 2000).

\section{Description of the intervention}

Low molecular weight heparins (LMWHs) do not have intrinsic anticoagulant activity but potentiate the activity of antithrombin III in inhibiting activated coagulation factors. These agents constitute indirect anticoagulants as their activity is mediated by plasma cofactors. LMWHs are not absorbed orally and must be administered parenterally by subcutaneous injections (Hirsh 1993).

Direct oral anticoagulant (DOACs) are a new generation of medications with a rapid onset of action that allows a fixed-dose treatment, and may simplify treatment of VTE by eliminating the need for an initial parenteral anticoagulation (Agnelli 2013).

Vitamin $\mathrm{K}$ antagonists (VKAs) have been the mainstay of oral anticoagulant therapy since the 1950s. Well-designed clinical trials have shown the effectiveness of VKAs for the primary and secondary prevention of several venous and arterial thrombotic diseases (Ansell 2008)

\section{How the intervention might work}

Several systematic reviews have compared LMWHs, DOACs and VKAs in the long-term treatment of VTE, but in populations not representative of people with cancer (Conti 2003; Iorio 2003; van der Heijden 2007). The review by van der Heijden and colleagues did not complete a preplanned subgroup analysis in people with cancer as the required data were not specifically reported (van der Heijden 2007). The review by Conti and colleagues did not conduct a metaanalysis in the subgroup of people with cancer (Conti 2003). In the review by lorio and colleagues, one meta-analysis in the subgroup of people with cancer found no significant difference in mortality (odds ratio 1.13 , 95\% confidence interval $(\mathrm{Cl}) 0.54$ to 2.38 ).

\section{Why it is important to do this review}

We initially conducted this and other reviews on this topic and their updates to directly and better inform clinical practice guidelines. This is fourth versions of this review (previous version published respectively in 2007, 2011, 2016 and cite them). The last major update of this Cochrane systematic review, published in 2018, identified 16 trials. It concluded that the LMWHs compared to VKAs probably produces an important reduction in VTE, and DOACS compared to LMWH may likely reduce VTE but may increase risk of major bleeding (Kahale 2018). Since 2018, we have identified three new eligible trials and one full-text publication of a previously identified abstract) addressing this question.

Living review approach: since the publication of the 2018 update of the review, we are maintaining it as a living systematic review. This means we will be continually running the searches and rapidly incorporating any newly identified evidence (for more information about the living systematic review approach, see Appendix 1). We believe a living systematic review approach is appropriate for this review for four reasons. First, the review addresses an important topic for clinical practice; people with cancer being treated for VTE have a relatively high rate of VTE recurrence. For instance, during oral anticoagulation therapy for VTE, people with cancer, compared with people without cancer, have a higher incidence of recurrent VTE (27.1 events per 100 participant-years with cancer versus 9.0 events per 100 participant-years without cancer; $P=0.003$ ) (Hutten 2000). Second, there remains uncertainty in the existing evidence in relation to the outcomes of mortality and bleeding. Third, we are aware of five ongoing eligible trials that will be important to incorporate in a timely manner. Fourth, this living systematic review may be used as part of a living guideline project (Akl 2017).

\section{O B JECT IVES}

- To compare the efficacy and safety of anthithrombotics including low molecular weight heparins (LMWHs), direct oral anticoagulants (DOACs), vitamin $\mathrm{K}$ antagonists (VKAs), and Idraparinuxfor the long-term treatment of venous thromboembolism (VTE) in people with cancer.

- To maintain this review as a living systematic review by continually running the searches and incorporating newly identified studies.

\section{METHODS}

\section{Criteria for considering studies for this review \\ Types of studies}

Randomized controlled trials (RCTs).

\section{Types of participants}

People with active cancer with a confirmed diagnosis of VTE (deep venous thrombosis (DVT) or pulmonary embolism (PE)). Participants could have been of any age group (including children), with either solid or hematologic cancer, at any cancer stage and irrespective of the type of cancer therapy. VTE should have been diagnosed using an objective diagnostic test.

Cancer should have been diagnosed by the time of inclusion in the study. 
We included studies with at least $75 \%$ of participants with a cancer status being active (i.e., we excluded studies with more than $25 \%$ of participants with non-active cancer). If between $25 \%$ and $75 \%$ of the population had active cancer and outcome data for this subgroup of people were not reported, we excluded such studies from the main analysis and included them in the sensitivity analysis.

\section{Types of interventions}

Intervention arms consisted of long-term treatment with antithrombtics including (beyond 3 months of initial treatment) :

- LMWHs;

- DOACs;

- VKAs;

- Idraparinux

We included any comparison of the three management options listed above (LMWHs versus VKAs, DOACs versus VKAs, DOACs versus LMWHs; idra). Cointerventions, if any, should have been balanced across the groups compared.

\section{Types of outcome measures}

\section{Primary outcomes}

- All-cause mortality.

\section{Secondary outcomes}

- Symptomatic recurrent DVT: DVT events suspected clinically, and confirmed using an objective diagnostic test such as: venography, 125 -fibrinogen-uptake test, impedance plethysmography or compression ultrasound.

- Symptomatic recurrent PE: PE events suspected clinically, and confirmed using an objective diagnostic test such as: pulmonary ventilation/perfusion scans, computed tomography, pulmonary angiography or autopsy.

- Symptomatic VTE:

- Major bleeding: we accepted the authors' definitions of major bleeding.

- Minor bleeding: we accepted the authors' definitions of minor bleeding.

- Thrombocytopenia: we accepted the authors' definitions of thrombocytopenia.

- Health-related quality of life measured using a validated tool.

- Postphlebetic syndrome.

\section{Search methods for identification of studies}

\section{Electronic searches}

The search was part of a comprehensive search for studies of anticoagulation in people with cancer. We conducted a comprehensive search on 14 May 2021, following the a major search in February 2016. We electronically searched the following databases: the Cochrane Central Register of Controlled Trials (CENTRAL), MEDLINE (starting 1946, via Ovid), and Embase (starting 1980, via Ovid). We used no language restrictions.The search strategies combined terms for anticoagulants, terms for cancer and a search filter for RCTs. We used no language restrictions. The search strategy was revised by an information specialist (JP). We list the full search strategiy for each of the electronic databases in Appendix 2.

Living systematic review approach: We will be updating the searches using auto-alerts on a monthly basis. We will publish an update every six months to incorporate any new identified evidence. This update of the systematic review is based on the findings of a literature search conducted on 14 May 2021. We will review search methods and strategies approximately yearly, to ensure they reflect any terminology changes in the topic area, or in the databases.

\section{Searching other resources}

We handsearched the conference proceedings of the American Society of Clinical Oncology (ASCO, starting with its first volume, 1982 up to May 2021) and of the American Society of Hematology (ASH, starting with its 2003 issue up to May 2021). We also searched ClinicalTrials.gov and World Health Organization (WHO) International Clinical Trials Registry Platform for ongoing studies. We reviewed the reference lists of papers included in this review and of other relevant systematic reviews.. In addition, we contacted experts in the field for information about unpublished work and ongoing trials.

Living review approach: we will search the conference proceedings of ASCO and ASH soon after their publications and ClinicalTrials.gov and WHO International Clinical Trials Registry Platform. . We will continue to review the reference lists for any prospectively identified studies specify in differences between protocol

\section{Data collection and analysis}

\section{Selection of studies}

Four pairs of review authors (LAK, FA, MBK, CFM, VEDY, IT, FS, MB, IGT) independently screened the title and abstract of identified articles for potential eligibility. We retrieved the full text of articles judged potentially eligible by at least one review author. Then, the pairs of authors independently screened the full-text article for eligibility using a standardized form piloted on 500 RCTs with explicit inclusion and exclusion criteria (as detailed in the Criteria for considering studies for this review section) and resolved any disagreements by discussion or by consulting a third review author.

Living systematic review approach: for the monthly searches, we will immediately screen the new citations retrieved each month. As the first step of monthly screening, we will apply the machine learning classifier (RCT model) available in the Cochrane Register of Studies (CSR-web; Wallace 2017). The classifier assigns a probability (from 0 to 100 ) to each citation for being a true RCT. For citations that are assigned a probability score of less than 10 , the machine learning classifier currently has a specificity/recall of 99.987\% (Thomas 2017; Wallace 2017)). For citations assigned a score from 10 to 100 , we will screen them in duplicate and independently. Citations that score 9 or less will be screened by Cochrane Crowd (Cochrane Crowd). Any citations that are deemed to be potential RCTs by Cochrane Crowd will be returned to the authors for screening.

\section{Data extraction and management}

Four pairs of review authors (LAK, FA, IT, IGT) independently extracted the data from each study and resolved any disagreements 
by discussion or by consulting a third review author. We aimed to collect data related to the following.

\section{Participants}

- Number of participants randomized to each study arm.

- Number of participants followed up in each study arm.

- Number of participants who discontinued treatment in each arm.

- Population characteristics (e.g., age, gender, co morbidities).

- Type of cancer (site,histology).

- Stage of cancer.

We defined active cancer as (1) non-squamous cell or basal cell invasive cancer diagnosed within 6 months before enrollment, (2) cancer treated within the previous 6 months, (3) recurrent or metastatic cancer, or (4) reported as active cancer during the study.

We extracted outcome data for people with active cancer. If the study included both people with active cancer and people with non-active cancer, we sought outcome data for the subgroup of people with active cancer.

\section{Interventions}

- Type of anticoagulant.(LMWH, VKA, or DOAC)

- Dosage or intensity of anticoagulation

- Duration of long-term and initial treatment

- Cointerventions including chemotherapy, target therapy, immunotherapy, radiation therapy, or a combination of these (type and duration).

\section{Outcomes}

We extracted both time-to-event data (for the mortality and recurrence of VTE outcomes) and dichotomous data (for all outcomes).

For time-to-event data, we abstracted the log (hazard ratio (HR)) and its variance from trial reports; if these were not reported, we digitized the published Kaplan-Meier survival curves and estimated the log (HR) and its variance using the method of Parmar (Parmar 1998). We also noted the minimum and maximum duration of follow-up, which were required to make these estimates. We performed these calculations in Stata 9 using a specially written program, which yielded the reported log (HR) and variance when used on the data presented in Table V of Parmar 1998.

For dichotomous data, we collected for each outcome and per arm number of events, number of participants randomized, and number of participants with incomplete data.

For the outcome major bleeding, we extracted events among people with gastrointestinal (GI) tract cancer and people without GI tract cancer. We further extracted major GI bleeding, major upper GI bleeding, major lower GI bleeding, and major non-GI bleeding.

We attempted to contact study authors for incompletely reported data. We decided a priori to consider abstracts in the main analysis only if authors supplied us with full reports of their methods and results, otherwise we included abstracts in the sensitivity analysis

None of the outcomes of interest were continuous variables.

\section{Other}

We extracted from each included trial any information on the following:

- Ethical approval;

- Source of funding;

- Conflict of interest.

\section{Assessment of risk of bias in included studies}

We assessed risk of bias at the study level using Cochrane's 'Risk of bias' tool (Higgins 2011). Four pairs of review authors ((LAK, FA, IT, IGT) independently assessed the methodologic quality of each included study and resolved any disagreements by discussion. Methodologic criteria included:

- Adequate sequence generation;

- Allocation concealment;

- Blinding of participants and personnel;

- Blinding of outcome assessment;

- Incomplete outcome data ;

- Selective outcome reporting;

- Other bias (e.g., whether the study was stopped early for benefit).

See the Dealing with missing data section about assessing risk of bias associated with participants with missing data per outcome and across studies.

We attempted to contact the authors for any study domain that was unclear. We re-evaluated our judgment when authors provided clarification.

\section{Measures of treatment effect}

We analyzed hazard ratio (HR) for time-to-event data and relative risk (RR) for dichotomous data, with 95\% confidence intervals (Cl). None of the outcomes of interest was meta-analyzed as a continuous variable.

\section{Unit of analysis issues}

The unit of analysis was the individual participant.

\section{Dealing with missing data}

\section{Identifying participants with missing data}

It was not clear whether certain categories of participants (e.g. those described as 'withdrew consent' or 'experienced adverse events') were actually followed up by the trial authors (versus had missing data) (Akl 2016). To identify participants with missing data, we followed the guidance suggested by Kahale et al (Kahale 2019) :

- Definitely not missing data: (1) participants explicitly reported as followed-up; (2) participants who died during the trial; (3) participants belonging to centres that were excluded.

- Definitely missing data: (1) participants explicitly reported as not followed up; (2) participants with unclear follow-up status and (a) excluded from the denominator of the analysis (i.e., complete case analysis); or (b) included in the denominator of the analysis and their outcomes were explicitly stated to be imputed. However, we did not treat them as missing data unless 
it is possible to obtain the number of observed/actual events (i.e., excluding imputed events) to avoid double counting.

- Potentially missing data: Participants with unclear follow-up status (e.g., included in the denominator of the analysis and their outcomes were not explicitly stated to be imputed).

\section{Dealing with participants with missing data in the primary meta-analysis}

In the primary meta-analysis, we used a complete-case analysis approach, i.e., we excluded participants considered to have missing data (Guyatt 2017; Kahale 2020).

For categorical data, we used the following calculations for each study arm:

- denominator: (number of participants randomized) - (number of participants definitely with missing data);

- numerator: number of participants with observed events (i.e. participants who experienced at least one event for the outcome of interest during their available follow-up time).

\section{Assessing the risk of bias associated with participants with missing data}

When the primary meta-analysis of a specific outcome found a statistically significant effect, we conducted sensitivity metaanalyses to assess the risk of bias associated with missing outcome data. Those sensitivity meta-analyses used a priori plausible assumptions about the outcomes of participants considered to have missing data. The assumptions we used in the sensitivity meta-analyses were increasingly stringent in order to challenge the statistical significance of the results of the primary analysis progressively (Akl 2013; Kahale 2020).

For categorical data and for an RR showing a reduction in effect $(\mathrm{RR}<1)$, we used the following increasingly stringent but plausible assumptions:

- For the control arm, relative incidence (RI) among those with missing data (lost to follow-up (LTFU)) compared with those with available data (followed up, FU) in the same arm (RI LTFU/FU) $=1$; for the intervention arm, $\mathrm{RI}_{\mathrm{LTFU} / \mathrm{FU}}=1.5$;

- For the control arm, $\mathrm{RI}_{\mathrm{LTFU} / \mathrm{FU}}=1$; for the intervention arm, $\mathrm{RI}_{\mathrm{LTFU} / \mathrm{FU}}=$ 2;

- For the control arm, $\mathrm{Rl}_{\mathrm{LTFU} / \mathrm{FU}}=1$; for the intervention arm, $\mathrm{Rl}_{\mathrm{LTFU} / \mathrm{FU}}=3$;

- For the control arm, $\mathrm{RI}_{\mathrm{LTFU} / \mathrm{FU}}=1$; for the intervention arm, RI LTFU/FU $=5$.

For RR showing an increase in effect $(R R>1)$, we switched the above assumptions between the control and interventions arms (i.e. used $\mathrm{RI}_{\mathrm{LTFU} / \mathrm{FU}}=1$ for the intervention arm).

Specifically, we used the following calculations for each study arm:

- denominator: (number of participants randomized)

- numerator: (number of participants with observed events) + (number of participants definitely with missing data with assumed events)
Assumed events are calculated by applying the a priori plausible assumptions to the participants definitely with missing data.

\section{Assessment of heterogeneity}

We assessed heterogeneity between trials by visual inspection of forest plots, estimation of the percentage heterogeneity between trials that could not be ascribed to sampling variation (12 test; Higgins 2011), and by a formal statistical test of the significance of the heterogeneity (Deeks 2001). If there was evidence of substantial heterogeneity, we investigated and reported the possible reasons for this (see section on Subgroup analysis and investigation of heterogeneity).

\section{Assessment of reporting biases}

We planned to create funnel plots for outcomes including 10 or more trials.

\section{Data synthesis}

For time-to-event data, we pooled the $\log (\mathrm{HRs})$ using a randomeffects model (DerSimonian 1986), and the generic inverse variance facility of Review Manager 5.4.1 (Review Manager 2020). For dichotomous data, we calculated the RR separately for each study. When analyzing data related to participants who were reported as non-compliant, we attempted to adhere to the principles of intention-to-treat (ITT) analysis. We approached the issue of noncompliance independently from that of missing data (Alshurafa 2012). We then pooled the results of the different studies using a random-effects model. We assessed the certainty evidence at the outcome level using the GRADE approach for each of the following comparisons and outcomes (GRADE Handbook):

- LMWH versus VKA

- DOAC versus VKA

- DOAC versus LMWH

- Idraparinux versus VKA

We included in the primary analysis all studies that provided outcome data for particpants with active cancer, and in the sensitivity analysis all studies that included between $25 \%$ and $75 \%$ of their study sample participants with active cancer and did not provide outcome data for this subgroup of people.

Living systematic review approach: whenever new evidence (studies, data or information) that meets the review inclusion criteria is identified, we will immediately assess risk of bias and extract the data and incorporate it in the synthesis, as appropriate. We will not adjust the meta-analyses to account for multiple testing given the methods related to frequent updating of meta-analyses are under development (Simmonds 2017).

\section{Subgroup analysis and investigation of heterogeneity}

Since anticoagulants might perfom differently in participants with GI tract cancer (CITATION), we planned to do a subgroup analysis for the outcome major bleeding among participants with GI tract cancer and those without GI tract cancer. We included studies that

- Recruited only patients with GI tract cancer and studies that recruited only participants with non-GI tract cancer; 
- Recruited both GI and non-GI tract cancer if they provided outcome data for subgroups of participants with $\mathrm{GI}$ tract cancer and data for subgroups of participants with non-GI tract cancer;

- Recruited both GI tract and non-GI tract cancer but did not provide subgroup data only if more than $75 \%$ of participants had GI tract cancer or more than $75 \%$ of participants had non-GI tract cancer.

If the proportion of participants with $\mathrm{Gl}$ tract cancer ranged between $25 \%$ and $75 \%$ and outcome data for this subgroup was not provided, we did not include such a study in the subgroup analysis.

For this subgroup analysis, we did not conduct complete case analysis for the primary analysis as noted under Dealing with missing data section. Instead we used the denominator reported in the analysis of each study report in order to balance numbers of participants with GI tract cancer and those without GI tract cancer.

\section{Sensitivity analysis}

We decided a priori to consider abstracts and completed studies published exclusively on ClinicalTrials.gov in the main analysis only if study authors supplied us with full reports of their methods and results; otherwise abstracts were included only in the sensitivity analysis.

As described earlier under Dealing with missing data section, we also planned for sensitivity meta-analyses to assess the risk of bias associated with missing outcome data when the primary metaanalysis of a specific outcome found a statistically significant effect.
In addition, we planned a sensitivity analysis including studies that included between $25 \%$ and $75 \%$ of their study sample participants with active cancer and did not provide outcome data for this subgroup of people.

\section{RES U L T S}

\section{Description of studies}

\section{Results of the search}

Figure 1 shows the study flow diagram. As of May 2021, the search strategy identified 3583 unique citations. The title and abstract screening identified 102 potentially eligible citations. The full-text screening of the full texts of these 102 citations identified 19eligible RCTs published as full reports (Agnelli 2015 (AMPLIFY); Agnelli 2020 (Caravaggio); Deitcher 2006 (ONCENOX); El Mokadem 2020; Hull 2006 (LITE); Lee 2003 (CLOT); Lee 2015 (CATCH); Lopez-Beret 2001; McBane 2019 (ADAM-VTE); Meyer 2002 (CANTHANOX); Prins 2014 (EINSTEIN); Raskob 2016 (HOKUSAI); Raskob 2018 (HOKUSAI); Romera 2009; Schulman 2015 (RECOVER I-II); van Doormaal 2010 (Van Gogh DVT trial); Young 2018 (SELECT-D)), and two studies published as abstracts (Cesarone 2003; Mazilu 2014 (OVIDIUS)). Since the last major update in May 2018, we included the full text of two previously identified ongoing studies (Agnelli 2020 (Caravaggio); McBane 2019 (ADAM-VTE), included the full text of a previously previously identified abstract (Young 2018 (SELECTD), included the full text of a new study (El Mokadem 2020). We identified five registered but unpublished trials: one terminated (Kamphuisen 2010 (Longheva)) and four ongoing (Kamphuisen 2010 (Longheva); Karatas 2015; Meyer 2016 (CASTA-DIVA); Ryun Park 2017 (PRIORITY); Schrag 2016 (CANVAS)). . 
Figure 1. Study flow diagram.

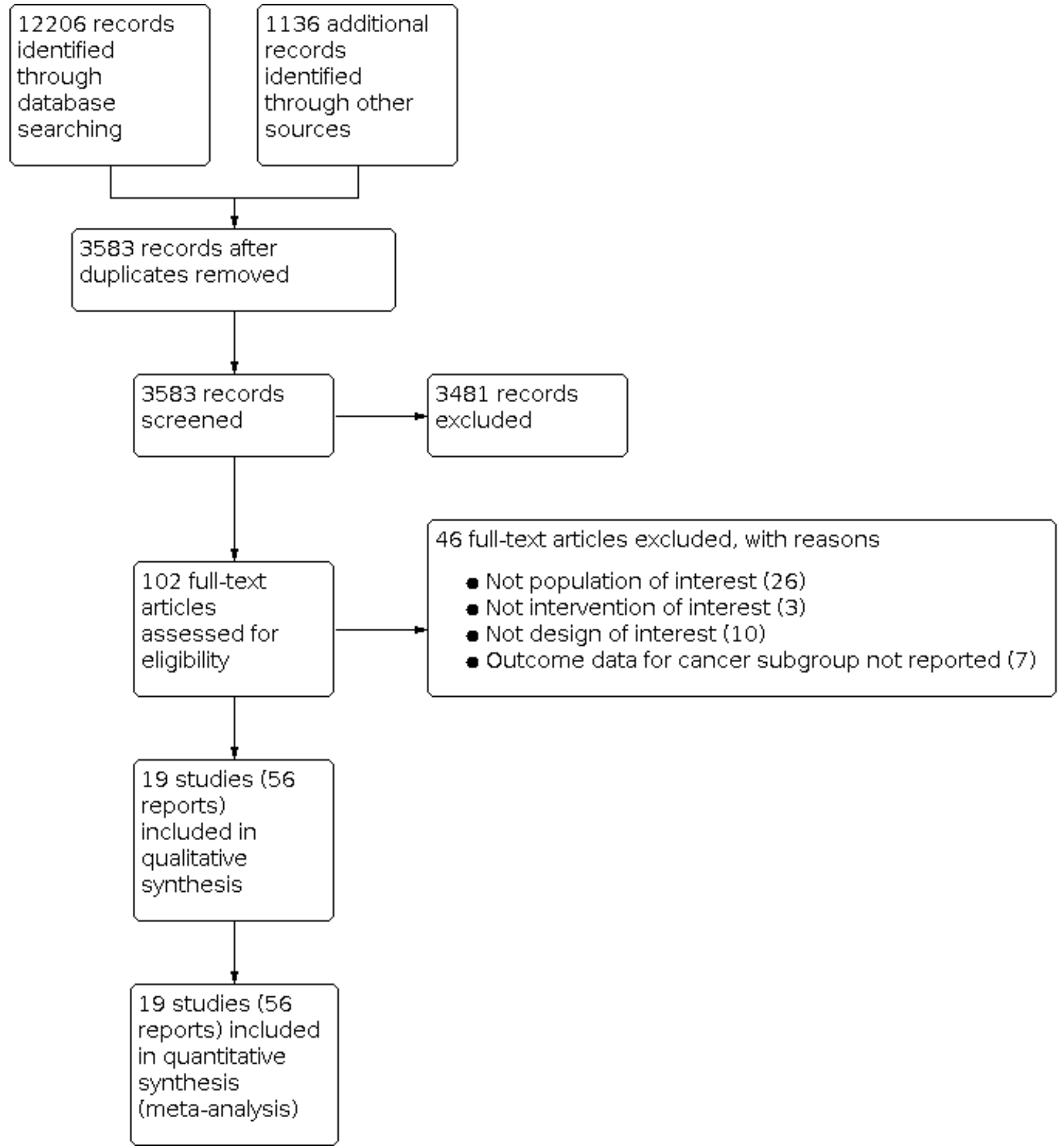

\section{Included studies}

We included 19 RCTs (55 reports) with participants with cancer for which outcome data were available (see Characteristics of included studies table).

Eight RCTs compared LMWHs to VKAs for the long-term treatment of VTE (Cesarone 2003; Deitcher 2006 (ONCENOX); Hull 2006 (LITE); Lee 2003 (CLOT); Lee 2015 (CATCH); Lopez-Beret 2001; Meyer 2002 (CANTHANOX); Romera 2009); only one of these studies used a different initial anticoagulant in the two study arms (LMWH in the
LMWH group and UFH in the VKA group) (Hull 2006 (LITE)). Four studies did not explictly specify whether patients had active cancer or did not provide outcome data for subgroups of patients with active cancer (Cesarone 2003; Hull 2006 (LITE); Lopez-Beret 2001; Romera 2009).

Five RCTs compared DOACs to VKAs (Agnelli 2015 (AMPLIFY); Mazilu 2014 (OVIDIUS); Prins 2014 (EINSTEIN); Raskob 2016 (HOKUSAI); Schulman 2015 (RECOVER I-II)). One study did not explictly specify whether patients had active cancer (Mazilu 2014 (OVIDIUS)). One RCT compared a once-weekly subcutaneous injection of 
idraparinux for three or six months versus standard treatment (tinzaparin, enoxaparin or dose-adjusted intravenous heparin followed by warfarin or acenocoumarol; van Doormaal 2010 (Van Gogh DVT trial)).

Five studies compared DOACs to LMWHs (Agnelli 2020 (Caravaggio); El Mokadem 2020; McBane 2019 (ADAM-VTE); Raskob 2018 (HOKUSAI); Young 2018 (SELECT-D)). We also identified five ongoing studies comparing DOACs to LMWHs (Kamphuisen 2010 (Longheva); Karatas 2015; Meyer 2016 (CASTA-DIVA); Ryun Park 2017 (PRIORITY); Schrag 2016 (CANVAS))).

Agnelli and colleagues recruited 169 participants with active cancer and VTE, a subgroup in the AMPLIFY trial, and followed them up for six months (Agnelli 2015 (AMPLIFY)). Participants were randomized to receive apixaban (10 $\mathrm{mg}$ twice daily for seven days followed by $5 \mathrm{mg}$ twice daily) or enoxaparin (1 $\mathrm{mg} \mathrm{kg}$ twice daily for at least five days) followed by dose-adjusted warfarin (target international normalized ratio (INR) of 2 to 3 ). Assessed outcomes were mortality, recurrent VTE and major bleeding. It is of note that 25 participants (13 in the apixaban group and 12 in the enoxaparin/warfarin group) without cancer or a history of cancer at baseline were diagnosed with cancer after treatment assignment.

Agnelli and colleagues recruited and analized 1155 participants with active cancer from 119 centers in 9 European countries, Israel and The U.S.A.(Agnelli 2020 (Caravaggio)). Participants were randomized to receive apixaban $10 \mathrm{mg}$ twice daily for seven days then $5 \mathrm{mg}$ twice daily for a total of six months or dalteparin 200 $\mathrm{IU} / \mathrm{kg}$ once daily for the first month and than $150 \mathrm{IU} / \mathrm{kg}$ daily for a total of six months. Assessed outcomes were all cause mortality, recurrent VTE, recurrent DVT, PE, major and minor bleeding. Gastrointestinal bleeding was also reported. Participants were followed up for six months.

Cesarone and colleagues recruited 199 participants with cancer and DVT (Cesarone 2003). The authors did not report whether the participants had active cancer or not. Participants were randomized to receive enoxaparin $100 \mathrm{Ul} / \mathrm{kg}$ twice daily or coumadin (dose adjusted to keep INR close to 3 ) for three months. Assessed outcomes were mortality and major outcome event in the three-month period.

Deitcher and colleagues recruited 102 participants with active cancer with acute symptomatic VTE (Deitcher 2006 (ONCENOX)). Participants were randomized to receive enoxaparin subcutaneous twice daily $(1.0 \mathrm{mg} / \mathrm{kg})$ for five days followed by once daily enoxaparin for 175 days or enoxaparin subcutaneous twice daily $(1.0 \mathrm{mg} / \mathrm{kg}$ ) for five days then warfarin starting day two of enoxaparin for 180 days. Assessed outcomes were mortality, recurrent VTE, and major and minor bleeding. .

El Mokadem and colleagues recruited and analyzed 138 participants with active cancer and acute DVT (El Mokadem 2020). Participants were randomized to receive apixaban $10 \mathrm{mg}$ twice daily for seven days then $5 \mathrm{mg}$ twice daily for a total of six months or enoxaparin $1 \mathrm{mg} / \mathrm{kg} / \mathrm{sc}$ every $12 \mathrm{~h}$ for a total of six months. Assessed outcomes were mortality, recurrence of DVT and PE, major bleeding, and minor bleeding. Participants were followed up for six months.

Hull and colleagues recruited 200 participants with cancer with acute symptomatic proximal vein thrombosis (Hull 2006 (LITE)).
Participants were randomized to receive tinzaparin 175 anti-Xa/kg subcutaneously daily for 12 weeks or UFH either $5000 \mathrm{U}$ or $80 \mathrm{U} /$ $\mathrm{kg}$ for five days followed by VKAs (target INR 2 to 3 ) for 12 weeks. Assessed outcomes were mortality, recurrent VTE, major and minor bleeding, and thrombocytopenia. Participants were followed up for one year.

Lee and colleagues recruited 676 participants with cancer and proximal DVT, PE or both in the CLOT study (Lee 2003 (CLOT)). Participants were randomized to receive dalteparin $200 \mathrm{IU}$ per kilogram once daily for five to seven days and a coumarin derivative for six months (target INR 2.5) or dalteparin alone for six months (200 IU per kilogram once daily for one month, followed by a daily dose of approximately $150 \mathrm{IU}$ per kilogram for five months). Assessed outcomes were mortality, recurrent VTE, and major and minor bleeding. Participants were followed up for six months.

Lee and colleagues recruited 900 participants with active cancer and objectively documented proximal DVT or PE in the CATCH study (Lee 2015 (CATCH)). Participants were randomized to receive tinzaparin $175 \mathrm{IU} / \mathrm{kg}$ once daily for six months or conventional therapy with tinzaparin $175 \mathrm{IU} / \mathrm{kg}$ once daily for five to 10 days followed by warfarin at a dose adjusted to maintain the INR within the therapeutic range ( 2 to 3 ) for six months. Assessed outcomes were mortality, recurrent VTE, and major and non-major bleeding. Participants were followed up for six months.

Lopez-Beret and colleagues recruited 35 participants with cancer and symptomatic DVT of the lower limb, a subgroup of 158 participants recruited (Lopez-Beret 2001a). Participants were randomized to receive nadroparin 1.025 anti-Xa IU/10 kg twice daily for three days then 1.025 anti-Xa IU/10 kg twice daily, after the third month, nadroparin was switched to once daily, or nadroparin 1.025 anti-Xa IU/10 kg twice daily for three days then acenocoumarol (target INR 2 to 3) for three to six months. Assessed outcome available for the cancer subgroup was mortality. Participants were followed up for 12 months.

Mazilu and colleagues recruited 46 participants with paraneoplastic DVT (Mazilu 2014 (OVIDIUS)). Participants were randomized to receive either fixed-dose dabigatran or adjusted-dose acenocoumarol. Assessed outcomes were mortality, recurrent VTE and bleeding.

McBane and colleagues recruited 300 participants with active cancer from 28 centers the U.S.A.(Mc Bane 2019 (ADAM-VTE)). Participants were randomized to receive apixaban $10 \mathrm{mg}$ twice daily for seven days then $5 \mathrm{mg}$ twice daily for a total of six months or dalteparin $200 \mathrm{lU} / \mathrm{kg}$ for the first month and than 150 $\mathrm{IU} / \mathrm{kg}$ once daily for a total of six months. Assessed outcomes were major bleeding, clinically-relevant non-major bleeding, any recurrence of DVT, PE, fatal PE, arterial thromboembolism and mortality.Gastrointestinal bleeding was also reported. Participants were followed up for six months.

Meyer and colleagues recruited 146 participants with cancer and VTE (Meyer 2002 (CANTHANOX)). Participants were randomized to receive enoxaparin $1.5 \mathrm{mg} / \mathrm{kg}$ daily for three months or enoxaparin $1.5 \mathrm{mg} / \mathrm{kg}$ daily for four days followed by warfarin (target INR 2 to 3) for three months. Outcomes assessed were mortality, recurrent VTE and major bleeding. Participants were followed up for three months. The study noted that $52 \%$ of participants had 
ongoing cancer treatment in the warfarin group versus $76 \%$ in the enoxaparin group.

Prins and colleagues recruited 459 participants with active cancer at baseline and DVT or PE, a subgroup of the EINSTEIN-DVT and EINSTEIN-PE studies (Prins 2014 (EINSTEIN)). Participants were randomized to receive rivaroxaban $15 \mathrm{mg}$ twice daily for 21 days, followed by $20 \mathrm{mg}$ once daily. Participants assigned to the enoxaparin and VKA group received enoxaparin subcutaneously $1.0 \mathrm{mg} / \mathrm{kg}$ bodyweight twice daily and either oral warfarin or acenocoumarol (target INR 2 to 3), started within 48 hours of randomization. Enoxaparin was discontinued when the INR was 2 or more for two days consecutively and the participant had received at least five days of enoxaparin treatment. The dose of the VKA was adjusted to maintain an INR of 2 to 3. Assessed outcomes were mortality, recurrent VTE, major bleeding and clinically relevant bleeding. Participants were followed up for 12 months.

Raskob and colleagues recruited 208 participants with active cancer and DVT or PE (Raskob 2016 (HOKUSAI)). Participants were randomized to receive LMWH for at least five days followed by oral edoxaban $60 \mathrm{mg}$ once daily (edoxaban group) or warfarin (or placebo) started concurrently with the study regimen of heparin. Assessed outcomes were recurrent VTE, major and non-major bleeding, and mortality. Participants were followed up for one year.

Raskob and colleagues recruited 1050 participants with active cancer and VTE (Raskob 2018 (HOKUSAI)). Participants were randomized to receive $\mathrm{LMWH}$ for at least five days followed by oral edoxaban $60 \mathrm{mg}$ once daily (edoxaban group) or subcutaneous dalteparin $200 \mathrm{IU}$ per kilogram bodyweight once daily for one month followed by dalteparin 150 IU per kilogram once daily (dalteparin group). Assessed outcomes were recurrent VTE, major and non-major bleeding, and mortality. Participants were followed up for one year.

Romera and colleagues recruited 69 participants with cancer with symptomatic proximal DVT, a subgroup of 241 recruited participants (Romera 2009). All participants were given tinzaparin fixed dose 175 IU anti-Xa per kg bodyweight once daily. The participants randomized to tinzaparin received this regimen for six months without dosage adjustments. The participants randomized to oral anticoagulants were given acenocoumarol $3 \mathrm{mg}$ orally, which was subsequently adjusted to achieve a regular INR between
2 and 3 for six months. This group received tinzaparin until the INR reached at least 2 on two consecutive measurements. The assessed outcome for the cancer subgroup was recurrent VTE. Participants were followed up for one year.

Schulman and colleagues recruited 221 participants with active cancer and VTE, a subgroup of the RECOVER and RECOVER-II trials (Schulman 2015 (RECOVER I-II)). Participants were randomized to receive warfarin adjusted to achieve an INR of 2 to 3 or dabigatran fixed-dose $150 \mathrm{mg}$ twice daily. In both randomization arms, initial treatment was with a parenteral anticoagulant (UFH, LMWH or fondaparinux) until the INR or sham INR became at least 2 for two consecutive days. Assessed outcomes were symptomatic recurrent VTE and VTE-related death, major bleeding and clinically relevant non-major bleeding. Participants were followed up for six months. The study authors reported complete follow-up.

Van Doormaal and colleagues recruited 284 participants with active cancer and DVT, a subgroup of the Van Gogh DVT trial (van Doormaal 2010 (Van Gogh DVT trial)). Participants were randomized to receive idraparinux for three or six months or VKA. The study noted that $66 \%$ of the idraparinux group and $69 \%$ of the VKAs group had active cancer. Assessed outcomes were mortality, recurrent VTE and bleeding. Participants were followed up for six months.

Young and colleagues recruited 406 participants with active cancer and VTE (Young 2017 (SELECT-D)). Participants were randomized to receive rivaroxaban $15 \mathrm{mg}$ twice daily for three weeks then $20 \mathrm{mg}$ once daily for a total of six months or dalteparin $200 \mathrm{IU} / \mathrm{kg}$ daily for one month and $150 \mathrm{IU} / \mathrm{kg}$ daily for a total of six months. Assessed outcomes were recurrent VTE, mortality, and major and clinically non-major bleeding. Gastrointestinal bleeding was also reported. Participants were followed up for six months.

\section{Excluded studies}

We excluded 46reports) from this review for the following reasons: not population of interest (26);not intervention of interest (3):; not design of interest (10) and outcome data for cancer subgroup not reported (7). See Characteristics of excluded studies table.

\section{Risk of bias in included studies}

The judgments for the risk of bias are summarized in Figure 2 and Figure 3.

\section{Figure 2. Risk of bias graph: review authors' judgments about each risk of bias item presented as percentages across all included studies.}

Random sequence generation (selection bias) Allocation concealment (selection bias) Blinding of participants and personnel (performance bias): All outcomes Blinding of outcome assessment (detection bias): All outcomes Incomplete outcome data (attrition bias): All outcomes Free of selective reporting? Free of other bias?
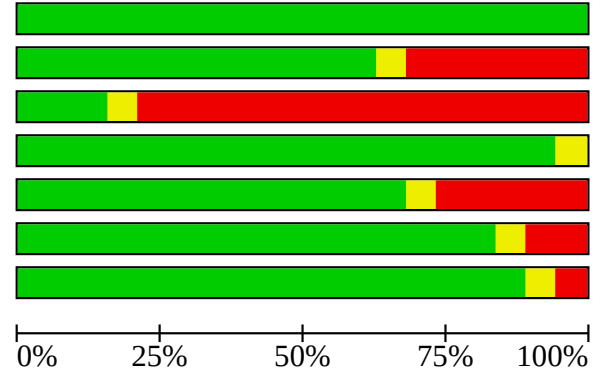
Figure 3. Risk of bias summary: review authors' judgments about each risk of bias item for each included study.

Agnelli 2015 (AMPLIFY)

Agnelli 2020 (Caravaggio)

Cesarone 2003

Deitcher 2006 (ONCENOX)

El Mokadem 2020

Hull 2006 (LITE)

Lee 2003 (CLOT)

Lee 2015 (CATCH)

Lopez-Beret 2001

Mazilu 2014 (OVIDIUS) McBane 2019 (ADAM-VTE)

Meyer 2002 (CANTHANOX)

Prins 2014 (EINSTEIN)

Raskob 2016 (HOKUSAI)

Raskob 2018 (HOKUSAI)

Romera 2009

Schulman 2015 (RECOVER I-II)

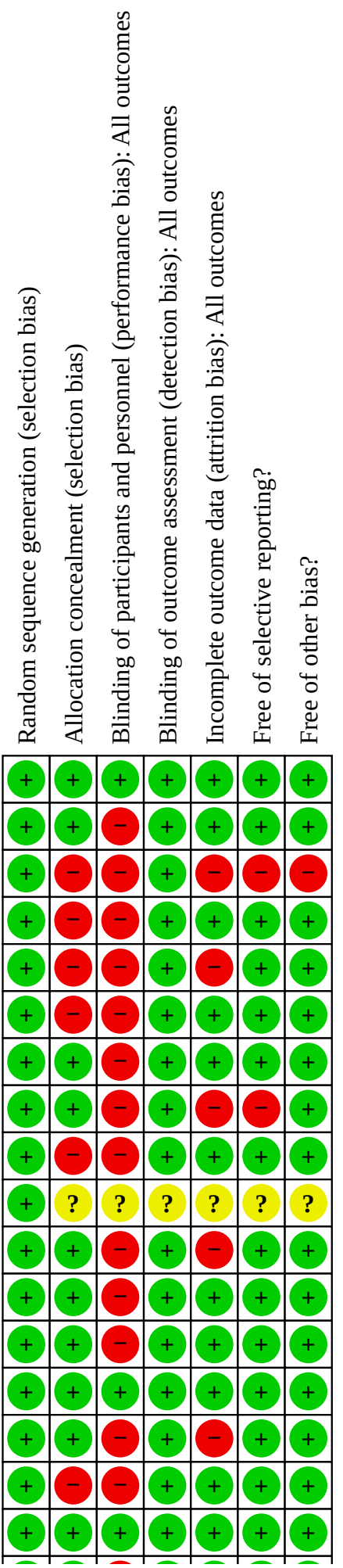


Figure 3. (Continued)

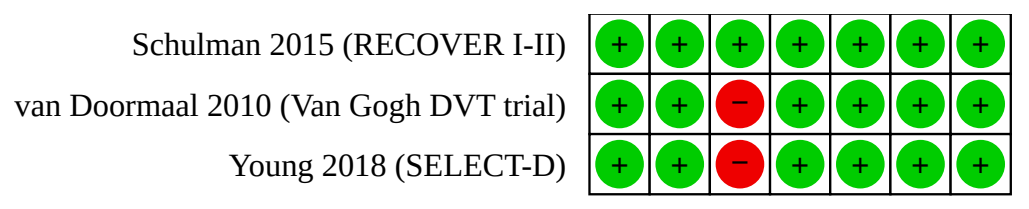

\begin{abstract}
Allocation
Random sequence was definitely generated in 14 studies (Agnelli 2015 (AMPLIFY); Agnelli 2020 (Caravaggio); El Mokadem 2020; Hull 2006 (LITE); Lee 2003 (CLOT); Lee 2015 (CATCH); McBane 2019 (ADAM-VTE); Meyer 2002 (CANTHANOX); Prins 2014 (EINSTEIN); Raskob 2016 (HOKUSAI); Raskob 2018 (HOKUSAI); Schulman 2015 (RECOVER I-II); van Doormaal 2010 (Van Gogh DVT trial); Young 2018 (SELECT-D)).
\end{abstract}

Five studies were judged to be at low risk of selection bias because minimal information about random sequence generation was provided (Cesarone 2003; Deitcher 2006 (ONCENOX); LopezBeret 2001; Mazilu 2014 (OVIDIUS); Romera 2009).

We judged allocation to be adequately concealed in 12 studies (Agnelli 2015 (AMPLIFY); Agnelli 2020 (Caravaggio) Lee 2003 (CLOT); Lee 2015 (CATCH); McBane 2019 (ADAM-VTE); Meyer 2002 (CANTHANOX); Prins 2014 (EINSTEIN); Raskob 2016 (HOKUSAI); Raskob 2018 (HOKUSAI); Schulman 2015 (RECOVER I-II); van Doormaal 2010 (Van Gogh DVT trial)). Eight studies did not report allocation concealment (Cesarone 2003; Deitcher 2006 (ONCENOX); El Mokadem 2020; Hull 2006 (LITE); Lopez-Beret 2001; Mazilu 2014 (OVIDIUS); Raskob 2018 (HOKUSAI); Romera 2009).

\section{Blinding}

\section{Blinding of participants and personnel (performance bias)}

We judged participants and personnel to be definitely blinded in four studies (Agnelli 2015 (AMPLIFY); Deitcher 2006 (ONCENOX); Raskob 2016 (HOKUSAI); Schulman 2015 (RECOVER I-II)), definitely not blinded in 11 studies (Agnelli 2020 (Caravaggio); Hull 2006 (LITE); Lee 2003 (CLOT); Lee 2015 (CATCH); Lopez-Beret 2001; Meyer 2002 (CANTHANOX); Prins 2014 (EINSTEIN); Raskob 2018 (HOKUSAI); Romera 2009; van Doormaal 2010 (Van Gogh DVT trial); Young 2018 (SELECT-D), and probably not blinded in three studies (Cesarone 2003; El Mokadem 2020; McBane 2019 (ADAM-VTE)). One study did not report on blinding of participants and personnel (unclear risk of bias; Mazilu 2014 (OVIDIUS)).

\section{Blinding of outcome assessment (detection bias)}

We judged outcome assessors to be definitely blinded in 10 studies (Agnelli 2015 (AMPLIFY); Agnelli 2020 (Caravaggio); Hull 2006 (LITE); Lee 2003 (CLOT); Lee 2015 (CATCH); Lopez-Beret 2001; Meyer 2002 (CANTHANOX); Prins 2014 (EINSTEIN); Raskob 2016 (HOKUSAI); Raskob 2018 (HOKUSAI); Romera 2009; Schulman 2015 (RECOVER III); van Doormaal 2010 (Van Gogh DVT trial)), definitely not blinded in one study (Young 2018 (SELECT-D)), and probably not blinded in four studies (Cesarone 2003; Deitcher 2006 (ONCENOX); El
Mokadem 2020; McBane 2019 (ADAM-VTE)) One study was not clear about the blinding of outcome assessors (Mazilu 2014 (OVIDIUS)).

\section{Incomplete outcome data}

We assessed the risk of bias associated with missing data for each outcome with a significant effect (please see Effects of interventions section). Seven studies did not report follow-up data for the cancer subgroup, but we assumed complete followup taking into consideration the small sample size (Agnelli 2015 (AMPLIFY); Lopez-Beret 2001; Prins 2014 (EINSTEIN); Raskob 2016 (HOKUSAI); Romera 2009; van Doormaal 2010 (Van Gogh DVT trial)) ).Six studies were judged to be at low risk of incomplete outcome data because the rates of missing outcome data were lower that the event rate in the studies (Agnelli 2020 (Caravaggio); Deitcher 2006 (ONCENOX); Hull 2006 (LITE); Lee 2003 (CLOT); Meyer 2002 (CANTHANOX); Young 2018 (SELECT-D)), whereas five studies were judged to be at high risk of incomplete outcome data because the rate of missing outcome data was higher that the event rate in the study (Cesarone 2003; El Mokadem 2020; Lee 2015 (CATCH); McBane 2019 (ADAM-VTE); Raskob 2018 (HOKUSAI)). One study reported complete follow-up based on communication with author( Schulman 2015 (RECOVER I-II)). The risk of incomplete outcome data was not clear in one study (Mazilu 2014 (OVIDIUS)).

\section{Selective reporting}

We did not suspect selective reporting of outcomes for any of the studies except for Cesarone 2003 where results for outcomes of interest were not reported individually, and all results were reported under the term "major outcome," in addition we suspected selective reporting in Lee 2015 (CATCH) where authors failed to report on some of the outcomes mentioned in the study protocol. The cancer subgroup data were missing for a large number of studies. Reporting bias was not clear in one study (Mazilu 2014 (OVIDIUS).

\section{Other potential sources of bias}

Another potential source of bias was the screening for asymptomatic VTE in three studies (Lopez-Beret 2001; Meyer 2002 (CANTHANOX); Romera 2009). The full-text of the abstract Cesarone 2003 was never published.

\section{Effects of interventions}

See: Summary of findings 1 Low molecular weight heparin secondary prophylaxis compared to vitamin $\mathrm{K}$ antagonist secondary prophylaxis in people with cancer with venous thromboembolism; Summary of findings 2 Direct oral anticoagulant secondary prophylaxis compared to Vitamin $K$ antagonist secondary prophylaxis in patients with active cancer with venous thromboembolism; Summary of findings 4 Direct oral anticoagulant secondary prophylaxis compared to Low 
molecular weight heparin secondary prophylaxis in patients with cancer with venous thromboembolism; Summary of findings 5 Idraparinux secondary prophylaxis compared to vitamin $\mathrm{K}$ antagonist secondary prophylaxis in people with cancer with venous thromboembolism

\section{Low molecular weight heparin versus vitamin $\mathrm{K}$ antagonist}

Seven RCTs compared LMWH to VKA for the long-term treatment of VTE in patients with cancer (Cesarone 2003; Deitcher 2006 (ONCENOX); Hull 2006 (LITE); Lee 2003 (CLOT); Lee 2015 (CATCH); Meyer 2002 (CANTHANOX); Romera 2009). The initial treatment was heparin in both arms for all the seven studies.

We identified one ongoing study comparing LMWH to VKA for the long-term treatment of cancer participants with VTE that was terminated due to slow inclusion of patients (Kamphuisen 2010 (Longheva)).

\section{All-cause mortality up to six months}

Meta-analysis of four RCTs including 1712 participants, found that LMWH may result in little to no difference on mortality up to six months when compared to VKA (Relative Risk (RR) $0.99,95 \% \mathrm{Cl}$ 0.88 to 1.13; Risk Difference (RD) 4 fewer per $1000,95 \%$ Cl 45 fewer to 45 more; $\left.\right|^{2}$ 0\%; low certainty evidence; Analysis 1.1) (Deitcher 2006 (ONCENOX); Lee 2003 (CLOT); Lee 2015 (CATCH); Meyer 2002 (CANTHANOX)). The certainty of evidence was rated down to low due to serious risk of bias and serious imprecision (Summary of findings 1 ).

The results were consistent in a sensitivity analysis including the study published as an abstract (RR $0.99,95 \% \mathrm{Cl} 0.88$ to 1.12 ) (Cesarone 2003),in a sensitvity analysis including the studies that did not specify whether the cancer was active in the included patients (RR 1.00, 95\% Cl 0.89 1.13) (Hull 2006 (LITE); Lopez-Beret 2001), and in a sensitivity analysis including the study that used a different initial anticoagulant in the two study arms (RR 1.00, 95\% Cl 0.89 to 1.12) (Hull 2006 (LITE)).

We did not create a funnel plot for the outcome of mortality due to the low number of included trials.

\section{All-cause mortality: time-to-event analysis}

Two studies including 810 participants reported data allowing their inclusion in the time-to-event analysis (Lee 2003 (CLOT); Meyer 2002 (CANTHANOX)). Meta-analysis indicated that LMWHmay reduce mortality slightly compared to VKA (HR 0.94, $95 \% \mathrm{Cl} 0.74$ to 1.20; RD 18 fewer per $1000,95 \% \mathrm{Cl} 81$ fewer to 56 more; $\mathrm{I}^{2} 16 \%$; low certainty evidence Analysis 1.2). The certainty of evidence was rated down to low due to serious risk of bias and serious imprecision (Summary of findings 1 ). The results were consistent in a sensitivity analysis including data provided by the author for the study that used a different initial anticoagulant in the two study arms (HR 0.96, 95\% $\mathrm{Cl} 0.81$ to 1.14 ) (Hull 2006 (LITE)).

\section{Recurrent venous thromboembolism up to six months}

None of the studies reported DVT and PE as separate outcomes. Meta-analysis of four studies including 956 participants found that LMWH probably reduces recurrent VTE up to six months compared to VKA (RR 0.59, 95\% Cl 0.44 to 0.80; RD 51 fewer per $1000,95 \% \mathrm{Cl}$ 69 fewer to 25 fewer; $120 \%$; moderate certainty evidence; Analysis 1.3) (Deitcher 2006 (ONCENOX); Lee 2003 (CLOT); Lee 2015 (CATCH);
Meyer 2002 (CANTHANOX)).The certainty of evidence was rated down to moderate due to serious risk of bias (Summary of findings 1).

The results were consistent in a sensitvity analysis including the studies that did not specify whether the cancer was active in the included patients (RR 0.59, 95\% Cl 0.45 to 0.79) (Hull 2006 (LITE); Romera 2009) and in a sensitivity analysis including the study that used a different initial anticoagulant in the two study arms (RR 0.56, $95 \% \mathrm{Cl} 0.42$ to 0.74 ) (Hull 2006 (LITE)).

Since the primary meta-analysis found a statistically significant effect, and in order to assess the risk of bias associated with missing data, we conducted sensitivity meta-analyses using the a priori plausible assumptions detailed in the Methods section. The effect estimate remained significant across all four stringent assumptions (Appendix 3).

We did not create a funnel plot for the outcome of recurrent VTE due to the low number of included trials.

\section{Recurrent venous thromboembolism: time-to-event analysis}

Two studies including 810 participants reported data allowing their inclusion in the time-to-event meta-analyses. We used timeto-event data reported by two studies (Lee 2003 (CLOT); Meyer 2002 (CANTHANOX)). Meta-analysis showed that LMWH probably reduces recurrent VTE (HR $0.49,95 \% \mathrm{Cl} 0.31$ to 0.78 ; RD 61 fewer per $1000,95 \% \mathrm{Cl} 84$ fewer to 26 fewer; $\left.\right|^{2} 0 \%$; moderate certainty evidence; Analysis 1.4).The certainty of evidence was rated down to moderate due to serious risk of bias (Summary of findings 1 ).

The results were consistent in a sensitivity analysis (Hull 2006 (LITE); Romera 2009) including data provided by the author for the study that used a different initial anticoagulant in the two study arms (HR 0.47, 95\% Cl 0.32 to 0.71) (Hull 2006 (LITE)).

We did not create a funnel plot for the outcome of recurrent VTE due to the low number of included trials.

\section{Major bleeding up to six months}

Meta-analysis of four studies including 1712 participants found than LMWH may result in little to no difference in major bleeding up tp six months compared to VKA (RR $1.09,95 \% \mathrm{Cl} 0.55$ to 2.12; RD 4 more per $1000,95 \% \mathrm{Cl} 19$ fewer to 48 more; $\left.\right|^{2}=46 \%$; low certainty evidence; Analysis 1.5) (Deitcher 2006 (ONCENOX); Lee 2003 (CLOT); Lee 2015 (CATCH); Meyer 2002 (CANTHANOX)). The certainty of evidence was rated down to low due to serious risk of bias and serious imprecision (Summary of findings 1 ).

The results were different in a sensitivity analysis including the study published as an abstract (RR $0.85,95 \% \mathrm{Cl} 0.41$ to 1.74 ) (Cesarone 2003) and in a senstivity analysis including the study that did not specify whether the cancer was active in the included patient and used a different initial anticoagulant in the two study arms for the outcome of major bleeding (RR 1.07, 95\% Cl 0.64 to 1.78) (Hull 2006 (LITE)).

We did not create a funnel plot for the outcome of major bleeding due to the low number of included trials. 


\section{Minor bleeding up to six months}

Meta-analysis of four studies including 1712 participants found than LMWH may reduce minor bleeding up tp six months compared to VKA but the evidence is very uncertain (RR $0.78,95 \% \mathrm{Cl} 0.47$ to 1.27 ; RD 38 fewer per $1000,95 \% \mathrm{Cl} 92$ fewer to 47 more; $1^{2}=78 \%$; very low certainty evidence; Analysis 1.6) (Deitcher 2006 (ONCENOX); Lee 2003 (CLOT); Lee 2015 (CATCH); Meyer 2002 (CANTHANOX)). The certainty of evidence was rated down to very low due to serious risk of bias, serious inconsistency, and serious imprecision (Summary of findings 1 ).

The results were consistent in a sensitivity analysis including the study that did not specify whether the cancer was active in the included patient and used a different initial anticoagulant in the two study arms (RR 0.84, 95\% Cl 0.56 to 1.27) (Hull 2006 (LITE)).

We did not create a funnel plot for the outcome of minor bleeding due to the low number of included trials.

\section{Thrombocytopenia up to six months}

One study including 138 participants assessed thrombocytopenia (Meyer 2002 (CANTHANOX)). The study found that LMWH may reduce thrombocytopenia up to six months slightly compared to VKA (RR 0.94, 95\% Cl 0.52 to 1.69; RD 15 fewer per 1000, 95\% Cl 122 fewer to 175 more; low certainty evidence; Analysis 1.7). The certainty of evidence was rated down to low due to very serious imprecision (Summary of findings 1 ).

The results were consistent in a sensitivity analysis including the study that did not specify whether the cancer was active in the included patient and used a different initial anticoagulant in the two study arms (RR 1.10, 95\% Cl 0.67 to 1.79) (Hull 2006 (LITE)).

We did not create a funnel plot for the outcome of thrombocytopenia due to the low number of included trials.

\section{Health-related quality of life up to six months}

None of the studies reported health-related quality of life.

\section{Postphlebitic syndrome up to six months}

None of the studies reported postphlebitic syndrome.

\section{Direct oral anticoagulants versus vitamin $\mathrm{K}$ antagonists}

Five RCTs compared DOAC to VKA for the long-term treatment of VTE in patients with cancer (Agnelli 2015 (AMPLIFY); Mazilu 2014 (OVIDIUS); Prins 2014 (EINSTEIN); Raskob 2016 (HOKUSAI); Schulman 2015 (RECOVER I-II)). The initial treatment was heparin in both arms for two studies (Raskob 2016 (HOKUSAI); Schulman 2015 (RECOVER I-II)), DOAC vs LMWH for two other studies (Agnelli 2015 (AMPLIFY); Prins 2014 (EINSTEIN), and DOAC vs VKA in one study (Mazilu 2014 (OVIDIUS).

\section{All-cause mortality six to 12 months}

Meta-analysis of five RCTs, including 1060 participants found that DOAC may reduce mortality from six to 12 months slightly compared to VKA (RR 0.94, 95\% Cl 0.72 to 1.23 ; RD 10 fewer per 1000 , $95 \% \mathrm{Cl} 48$ fewer to 39 more; $12=0 \%$; low certainty evidence; Analysis 2.1) (Agnelli 2015 (AMPLIFY); Prins 2014 (EINSTEIN); Raskob 2016 (HOKUSAI); Schulman 2015 (RECOVER I-II)). The certainty of evidence was rated down to low due to very serious imprecision (Summary of findings 2).

The results were consistent in a sensitivity analysis including the study published as an abstract and that did not specify whether the cancer was active in the included patients (RR $0.92,95 \% \mathrm{Cl} 0.71$ to 1.19) (Mazilu 2014 (OVIDIUS)).

We did not create a funnel plot for the outcome of all-cause mortality due to the low number of included trials.

\section{Recurrent venous thromboembolism six to 12 months}

None of the studies reported DVT and PE as separate outcomes. Meta-analysis of four studies including 1050 participants found that DOAC may reduce recurrent VTE from six to to 12 months slightly compared to VKA (RR $0.63,95 \% \mathrm{Cl} 0.34$ to 1.15 ; RD 18 fewer per $1000,95 \% \mathrm{Cl} 32$ fewer to 7 more; $\mathrm{I}^{2}=0 \%$, low certainty evidence; Analysis 2.2) (Agnelli 2015 (AMPLIFY); Prins 2014 (EINSTEIN); Raskob 2016 (HOKUSAI); Schulman 2015 (RECOVER III)). The certainty of evidence was rated down to low due to very serious imprecision (Summary of findings 2).

We did not create a funnel plot for the outcome of recurrent VTE due to the low number of included trials.

\section{Major bleeding six to 12 months}

Meta-analysis of four studies including 1055 participants found that DOACs may result in little to no difference in major bleeding compared to VKA (RR $0.77,95 \% \mathrm{Cl} 0.39$ to 1.53 ; RD 8 fewer per 1000 , $95 \% \mathrm{Cl} 23$ fewer to 20 more; low certainty evidence; $12=0 \%$; Analysis 2.3) (Agnelli 2015 (AMPLIFY); Prins 2014 (EINSTEIN); Raskob 2016 (HOKUSAI); Schulman 2015 (RECOVER I-II)).The certainty of evidence was rated down to low due to very serious imprecision (Summary of findings 2).

We did not create a funnel plot for the outcome of major bleeding due to the low number of included trials.

\section{Minor bleeding six to 12 months}

Meta-analysis of four studies including 1055 participants found that DOACs may reduce minor bleeding from six to to 12 months slightly compared to VKA (RR $0.83,95 \% \mathrm{Cl} 0.57$ to 1.23 ; RD 22 fewer per $1000,95 \% \mathrm{Cl} 55$ fewer to 29 more; low-certainty evidence; Analysis 2.4) (Agnelli 2015 (AMPLIFY); Prins 2014 (EINSTEIN); Raskob 2016 (HOKUSAI); Schulman 2015 (RECOVER I-II)). The certainty of evidence was rated down to low due to very serious imprecision (Summary of findings 2).

We did not create a funnel plot for the outcome of minor bleeding due to the low number of included trials.

\section{Thrombocytopenia six to 12 months}

None of the studies reported thrombocytopenia.

\section{Health-related quality of life six to 12 months}

Two studies assessed health-related quality of life; the first used the Anti-Clot Treatment Scale (ACTS) (Prins 2014 (EINSTEIN)), while the other did not report the tool used for assessment (Mazilu 2014 (OVIDIUS)). Prins and colleagues assessed the outcome for the study population (8485 participants) without reporting on the cancer subgroup (655 participants). They reported that HRQoL 
was better in the rivaroxaban-treated participants than in the group treated with enoxaparin and VKAs (no further statistical data reported). The certainty of evidence was rated down to low due to very serious imprecision (Summary of findings 2 ).

The study by Mazilu and colleagues, published as an abstract, reported that HRQoL was better in the dabigatran group due to the fact that there was no need for monthly blood tests as in the acenocoumarol group (Mazilu 2014 (OVIDIUS)).

\section{Postphlebitic syndrome}

None of the studies reported postphlebitic syndrome.

\section{Direct oral anticoagulants versus low molecular weight heparins}

Five RCTs compared DOAC to LMWH for the long-term treatment of VTE in patients with cancer (Agnelli 2020 (Caravaggio); El Mokadem 2020; McBane 2019 (ADAM-VTE); Raskob 2018 (HOKUSAI); Young 2018 (SELECT-D)). The initial treatment was DOAC vs LMWH for four studies (Agnelli 2020 (Caravaggio); El Mokadem 2020; McBane 2019 (ADAM-VTE); Young 2018 (SELECT-D)) and heparin in both arms for one study (Raskob 2018 (HOKUSAI)).

We identified four ongoing studies comparing DOACs to LMWHs for the long-term treatment of cancer participants with VTE;two are completed and not published yet (Meyer 2016 (CASTA-DIVA); Schrag 2016 (CANVAS)); one is still recruiting (Ryun Park 2017 (PRIORITY)); and one ongoing study that was terminated since recruitment was not as expected (Karatas 2015).

\section{All-cause mortality up to six months}

Meta-analysis of five RCTs, including 2854 participants found that DOAC may result in little to no difference in mortality up to six months compared to LMWH (RR $0.97,95 \% \mathrm{Cl} 0.84$ to 1.14; RD 7 fewer per $1000,95 \% \mathrm{Cl} 42$ fewer to 35 more; $\mathrm{I}^{2}=25 \%$; low certainty evidence; Analysis 3.1) (Agnelli 2020 (Caravaggio); El Mokadem 2020; McBane 2019 (ADAM-VTE); Raskob 2018 (HOKUSAI); Young 2018 (SELECT-D)). The certainty of evidence was rated down to low due to serious risk of bias and serious imprecision (Summary of findings 4).

We did not create a funnel plot for the outcome of all cause mortality due to the low number of included trials.

\section{Recurrent venous thromboembolism up to six months}

Meta-analysis of five RCTs, including 2854 participants found that DOAC may reduce recurrent VTE up to six months compared to LMWH (RR 0.63, 95\% Cl 0.45 to 0.88; RD 32 fewer per 1000, 95\% $\mathrm{Cl} 48$ fewer to 10 more; $\mathrm{I}^{2}=18 \%$; low certainty evidence; Analysis 3.2) ) (Agnelli 2020 (Caravaggio); El Mokadem 2020; McBane 2019 (ADAM-VTE); Raskob 2018 (HOKUSAI); Young 2018 (SELECT-D)). The certainty of evidence was rated down to low due to serious risk of bias and serious imprecision (Summary of findings 4).

We did not create a funnel plot for the outcome of recurrent VTE due to the low number of included trials.

Since the primary meta-analysis found a statistically significant effect, and in order to assess the risk of bias associated with missing participant data, we conducted sensitivity meta-analyses using the a priori plausible assumptions detailed in the Methods section.
The effect estimate remained significant across all four stringent assumptions (Appendix 3).

\section{Major bleeding up to six months}

Meta-analysis of five RCTs, including 2854 participants found that DOAC may result in little to no difference in major bleeding up to six months compared to LMWH (RR 1.20, 95\% Cl 0.83 to 1.73; RD 7 more per $1000,95 \% \mathrm{Cl} 6$ fewer to 25 more; $\mathrm{I}^{2}=18 \%$; low certainty evidence; Analysis 3.3) (Agnelli 2020 (Caravaggio); El Mokadem 2020; McBane 2019 (ADAM-VTE); Raskob 2018 (HOKUSAI); Young 2018 (SELECT-D)). The certainty of evidence was rated down to low due to serious risk of bias and serious imprecision (Summary of findings 4). A subgroup analysis of participants with GI tract cancer versus participants without $\mathrm{GI}$ tract cancer found no subgroup difference (among people with GI tract cancer RR 1.47, 95\% Cl 0.76 to 2.84; among people without $\mathrm{GI}$ tract cancer RR $1.05,95 \% \mathrm{Cl}$ 0.63 to 1.73) (Analysis 3.3) ((Agnelli 2020 (Caravaggio); El Mokadem 2020; Raskob 2018 (HOKUSAI); Young 2018 (SELECT-D)).

Meta-analysis of four RCTs, including 1838 participants found that DOAC may result in little to no difference in major $\mathrm{Gl}$ tract bleeding up to six months compared to LMWH (RR $1.16,95 \% \mathrm{Cl} 0.62$ to 2.17; RD 3 more per $1000,95 \% \mathrm{Cl} 8$ fewer to 23 more; $\mathrm{I}^{2}=0 \%$; low certainty evidence; Analysis 3.4) (Agnelli 2020 (Caravaggio); El Mokadem 2020; McBane 2019 (ADAM-VTE); Raskob 2018 (HOKUSAI); Young 2018 (SELECT-D)). The certainty of evidence was rated down to low due to serious risk of bias and serious imprecision (Summary of findings 4).

Meta-analysis of four RCTs, including 1838 participants found that DOAC may have little to no difference in major upper GI tract bleeding up to six months compared to LMWH but the evidence is very uncertain ( $\mathrm{RR} 1.18,95 \% \mathrm{Cl} 0.51$ to 2.76 ; RD 2 more per $1000,95 \% \mathrm{Cl} 5$ fewer to 19 more; $\mathrm{I}^{2}=0 \%$; very low certainty evidence; Analysis 3.5) (Agnelli 2020 (Caravaggio); El Mokadem 2020; McBane 2019 (ADAM-VTE); Young 2018 (SELECT-D)). The certainty of evidence was rated down to very low due to serious risk of bias and very serious imprecision (Summary of findings 4).

Meta-analysis of four RCTs, including 1838 participants found that DOAC may have little to no difference in major lower GI tract bleeding up to six months compared to LMWH but the evidence is very uncertain ( $\mathrm{RR} 1.10,95 \% \mathrm{Cl} 0.43$ to 2.80 ; $\mathrm{RD} 1$ more per $1000,95 \% \mathrm{Cl} 5$ fewer to 16 more; $\mathrm{I}^{2}=0 \%$; very low certainty evidence;Analysis 3.6) (Agnelli 2020 (Caravaggio); El Mokadem 2020; McBane 2019 (ADAM-VTE); Young 2018 (SELECT-D)). The certainty of evidence was rated down to very low due to serious risk of bias and very serious imprecision (Summary of findings 4).

Meta-analysis of four RCTs, including 1838 participants found that DOAC may have little to no difference in major non-GI tract bleeding up to six months compared to LMWH but the evidence is very uncertain (RR $0.84,95 \% \mathrm{Cl} 0.62$ to 1.68; RD 3 fewer per $1000,95 \% \mathrm{Cl}$ 11 fewer to 13 more; $1^{2}=0 \%$; very low certainty evidence;Analysis 3.7) (Agnelli 2020 (Caravaggio); El Mokadem 2020; McBane 2019 (ADAM-VTE); Young 2018 (SELECT-D)). The certainty of evidence was rated down to very low due to serious risk of bias and very serious imprecision (Summary of findings 4).

We did not create a funnel plot for the outcome of recurrent VTE due to the low number of included trials. 


\section{Minor bleeding up to six months}

Meta-analysis of five RCTs, including 2854 participants found that DOAC probably reduces minor bleeding up to six months compared to LMWH (RR 1.58, 95\% Cl 1.15 to 2.16; RD 39 more per $1000,95 \% \mathrm{Cl}$ 10 more to 78 more; $\mathrm{I}^{2}=23 \%$; moderate certainty evidence; Analysis 3.8) (Agnelli 2020 (Caravaggio); El Mokadem 2020; McBane 2019 (ADAM-VTE); Raskob 2018 (HOKUSAI); Young 2018 (SELECT-D)).The certainty of evidence was rated down to moderate due to serious risk of bias (Summary of findings 4).

Since the primary meta-analysis found a statistically significant effect, and in order to assess the risk of bias associated with missing data, we conducted sensitivity meta-analyses using the a priori plausible assumptions detailed in the Methods section. The effect estimate lost significance with RI 3 and RI 5, indicating high risk of bias associated with missing data (Appendix 3 ).

Meta-analysis of two RCTs, including 1495 participants found that DOAC may have little to no difference in minor GI tract bleeding up to six months compared to LMWH but the evidence is very uncertain (RR 1.37, $95 \% \mathrm{Cl} 0.41$ to 4.64; RD 9 more per $1000,95 \% \mathrm{Cl} 15$ fewer to 92 more; $I^{2}=64 \%$; very low certainty evidence; Analysis 3.9) (Agnelli 2020 (Caravaggio); Young 2018 (SELECT-D)). The certainty of evidence was rated down to very low due to serious inconsistency and very serious imprecision (Summary of findings 4).

Meta-analysis of two RCTs, including 1495 participants found that DOAC may have little to no difference in minor upper GI tract bleeding up to six months compared to LMWH but the evidence is very uncertain ( $\mathrm{RR} 1.03,95 \% \mathrm{Cl} 0.04$ to 25.97 ; $\mathrm{RD} 0$ more per $1000,95 \% \mathrm{Cl} 10$ fewer to 267 more; $\mathrm{I}^{2}=74 \%$; very low certainty evidence; Analysis 3.10) (Agnelli 2020 (Caravaggio); Young 2018 (SELECT-D)). The certainty of evidence was rated down to very low due to very serious inconsistency and very serious imprecision (Summary of findings 4).

Meta-analysis of two RCTs, including 1495 participants found that DOAC may have little to no difference in minor lower GI tract bleeding up to six months compared to LMWH (RR 1.54, 95\% Cl 0.72 to 3.27 ; RD 8 more per $1000,95 \% \mathrm{Cl} 4$ fewer to 33 more; $1^{2}=0 \%$; low certainty evidence; Analysis 3.11) (Agnelli 2020 (Caravaggio); Young 2018 (SELECT-D)). The certainty of evidence was rated down to low due to very serious imprecision (Summary of findings 4).

Meta-analysis of two RCTs, including 1495 participants found that DOAC probably increases minor non- GI tract bleeding up to six months compared to LMWH (RR 2.37, 95\% Cl 1.44 to 3.89; RD 42 more per $1000,95 \% \mathrm{Cl} 18$ fewer to 89 more; $\mathrm{I}^{2}=3 \%$; moderate certainty evidence; Analysis 3.12) (Agnelli 2020 (Caravaggio); Young 2018 (SELECT-D)). The certainty of evidence was rated down to moderate due to serious imprecision (Summary of findings 4).

\section{Thrombocytopenia up to six months}

None of the studies reported thrombocytopenia.

\section{Health-related quality of life up to six months}

None of the studies reported HRQoL.

\section{Postphlebitic syndrome up to six months}

None of the studies reported postphlebitic syndrome.

\section{Once-weekly idraparinux versus vitamin $\mathrm{K}$ antagonists}

One RCT with 284 participants compared once-weekly subcutaneous injection of idraparinux versus standard treatment (parenteral anticoagulation followed by warfarin or acenocoumarol) for three or six months (van Doormaal 2010 (Van Gogh DVT trial)).

\section{All-cause mortality up to six months}

The trial found thatidraparinux may reduce all-cause mortality up to six months slightly compared to VKAs on mortality at six months (RR 1.11, 95\% Cl 0.78 to 1.59; RD 31 more per 1000 , 95\% Cl 62 fewer to 167 more; low-certainty evidence).

\section{Recurrent venous thromboembolism up to six months}

The trial found that idraparinux may reduce recurrent VTE up to six months slightly compared to VKA (RR $0.46,95 \% \mathrm{Cl} 0.16$ to 1.32 ; RD 42 fewer per $1000,95 \%$ Cl 65 fewer to 25 more; low-certainty evidence).

\section{Major bleeding up to six months}

The trial found that idraparinux may result in little to no difference in major bleeding up to six months slightly compared to VKA (RR $1.11,95 \% \mathrm{Cl} 0.35$ to 3.56 ; RD 4 more per $1000,95 \% \mathrm{Cl} 25$ fewer to 98 more; low-certainty evidence).

\section{Minor bleeding up to six months}

The study did not report minor bleeding.

\section{Thrombocytopenia up to six months}

The study did not report thrombocytopenia.

\section{Health-related quality of life up to six months}

The study did not report HRQoL.

\section{Postphlebitic syndrome}

The study did not report postphlebitic syndrome.

We did not create funnel plots due to the low number of included trials for each outcome.

\section{DISCUSSION}

\section{Summary of main results}

For the long-term treatment of VTE in people with cancer, LMWH compared to VKAmay result in little to no difference on mortality and major bleeding up to six months, probably reduces recurrent VTE up to six months, may reduce minor bleeding up tp six months but the evidence is very uncertain, and may reduce thrombocytopenia up to six months slightly.

Direct oral anticoagulants compared to VKA may reduce mortality, recurrent VTE from six to 12 months slightly and may result in little to no difference in major bleeding compared to VKA.

Direct oral anticoagulants compared to LMWH may result in little to no difference in mortality, major bleeding, major GI tract bleeding, up to six months, may reduce recurrent VTE up to six months, may have little to no difference in major upper $\mathrm{GI}$ tract bleeding, major lower GI tract bleeding, major non-GI tract bleeding, minor GI tract 
bleeding, minor upper $\mathrm{Gl}$ tract bleeding, but the evidence is very uncertain, probably reduces minor bleeding up to six months, may have little to no difference in minor lower GI tract bleeding, and probably increases minor non- Gl tract bleeding up to six months

Idraparinux compared to VKA may reduce all-cause mortality, recurrent VTE, up to six months slightly, and may result in little to no difference in major bleeding up to six months slightly.

\section{Overall completeness and applicability of evidence}

While the reduction in VTE with LMWHs was expected to reduce thrombosis-related mortality, this did not translate into an observed reduction in all-cause mortality. This finding was not apparently explained by an increase in any specific-cause mortality (e.g. fatal bleeding), but might have been due to the lack of power to detect a reduction in all-cause mortality. Similarly, the size of the available evidence was not large enough to rule out beneficial or harmful effects for many comparisons (e.g. effects of LMWHs versus VKAs on bleeding, effects of DOACs vs VKA on bleeding, effects of DOACs vs LMWH on mortality, bleeding).

Stratifying bleeding outcomes based on type of cancer (GI tract vs non-GI tract) did not find any subgroup effect when comparing DOAC to LWMH. When adding patients with non-active cancer (or unclear whether active cancer) to patients with active cancer, the results of the meta-analyses for all studied outcomes remained unchanged. were unable to conduct subgroup analyses based on histologic type (GI tract) or stage of cancer (active) because of the lack of data DELETE ONCE CONFIRMED BY EA. In the absence of evidence for the contrary, we assumed that the results of this review applied to people with any type or stage of cancer.

For comparisons including DOACs, four RCTs used Apixaban (Agnelli 2015 (AMPLIFY); Agnelli 2020 (Caravaggio); El Mokadem 2020; McBane 2019 (ADAM-VTE)); three RCTs used Rivaroxaban (Prins 2014 (EINSTEIN); Raskob 2016 (HOKUSAI); Raskob 2018 (HOKUSAI); two RCTs used Edoxaban (Raskob 2016 (HOKUSAI); Raskob 2018 (HOKUSAI)) raskob 20162018 ; dabigatran mazilu schulman we were unable to conduct subgroup analyses based on different agents and hence we cannot make any judgment in related to class effect. The majority of studies used Apixaban CHARBEL, SOME LITERATURE ON CLASS EFFECT

majority of stufies used apixaban - unclear evidence regarding edoaxaban or other classess of DPAC

\section{Quality of the evidence}

Our systematic approach to searching, study selection and data extraction should have minimized the likelihood of missing relevant studies.

When comparing LMWHs to VKAs, we judged the certainty of evidence to be moderate for recurrent VTE due to serious risk of bias, and moderate for mortality at one year, and major bleeding due to both imprecision and risk of bias and low for minor bleeding due to imprecision, risk of bias and inconsistency..

We downgraded recurrent VTE by one level due to serious risk of bias, allocation concealment unclear in two studies, high risk of selective reporting and high risk of incomplete outcome data in one study, and lack of blinding of participants and personnel in all included studies. We downgraded the outcomes of mortality and major bleeding by one level due to both risk of bias and imprecision, in addition we downgraded the outcome of minor bleed by two levels, one for inconsistency and one for risk of bias and imprecision combined. The lack of allocation concealment in two of the studies did not affect the results when conducting a sensitivity analysis after removing those studies that had a combined weight of $6.5 \%$, but we were concerned about the lack of blinding of participants and personnel in all included studies in addition to high risk of bias in the CATCH trial that represented $43.1 \%$ of the weight, so we decided to downgrade by one level due to both concerns about imprecision and risk of bias.

When comparing DOACs to VKAs, we judged the certainty of evidence to be moderate for HRQoL due to serious indirectness, low for mortality, recurrent VTE, and major and minor bleeding due to serious imprecision and serious indirectness.

When comparing DOACs to LMWHs, we judged the certainty of evidence to be low for mortality, VTE recurrence, and major and minor bleeding due to serious risk of bias and serious imprecision.

When comparing idraparinux to VKAs, we judged the certainty of evidence to be moderate for mortality due to serious imprecision and low for recurrent VTE and major bleeding due to very serious imprecision, Taking into consideration the wide $\mathrm{Cls}$, the low number of events and the fact that only one study is providing data for this comparison.pote

\section{Potential biases in the review process}

The inclusion of different types of cancer in the same study precluded us from conducting the subgroup analyses to explore effect modifiers such as type and stage of cancer. The interpretation of findings was also limited by not including data from the trials published as abstracts only. A potential bias of our review might be the limitation of the electronic search strategy to participants with cancer, while the data needed for this review came from studies not restricted to this subgroup. Also, there might be potential bias associated with multiple testing in the planned meta-analyses and currently there are no plans to adjust meta-analyses for multiple testing. A major limitation of this review was that we were unable to include in the meta-analyses 11 eligible RCTs with subgroups of participants with cancer because relevant data were not reported and not obtainable from the authors.

\section{Agreements and disagreements with other studies or reviews}

We identified seven published systematic reviews comparing LMWHs or DOACs to VKAs in the long-term treatment of VTE (Conti 2003; Iorio 2003; Laporte 2011; Noble 2008; Posch 2015; RomeraVillegas 2010; Vedovati 2015). We review below the findings of the two most recent reviews.

Posch and colleagues compared LMWHs or DOACs to VKAs for the long-term treatment of VTE in participants with cancer including six RCTs comparing LMWHs to VKAs and four RCTs comparing DOACs to VKAs (Posch 2015). The meta-analysis found a significant reduction of recurrent VTE in favor of LMWHs (RR $0.6,95 \% \mathrm{Cl} 0.45$ to 0.79 ) and a non-significant difference in major bleeding episodes (RR 1.07, $95 \% \mathrm{Cl} 0.66$ to $1.73 ; \mathrm{p}=0.80$ ). There was no significant difference in recurrent VTE and major bleeding when comparing DOACs to VKAs 
(recurrent VTE: RR $0.65,95 \% \mathrm{Cl} 0.38$ to 1.09 ; major bleeding: RR $0.72,95 \% \mathrm{Cl} 0.39$ to 1.35$)$. These results were in agreement with our study.

Vedovati and colleagues compared DOACs to VKAs in the longterm treatment of VTE in participants with cancer (Vedovati 2015). Meta-analysis of five RCTs showed no significant difference in VTE recurrence when comparing DOACs to VKAs (RR $0.63,95 \% \mathrm{Cl} 0.37$ to 1.1). These results were in agreement with our study.

\section{AUTHORS' CONCLUSIONS}

\section{Implications for practice}

The decision for a person with cancer and venous thromboembolism (VTE) to start long-term low molecular weight heparin (LMWHs) treatment or oral anticoagulation treatment should balance the benefits and harms and integrate people's values and preferences for outcomes and management options (Haynes 2002). While DOACs compared to LMWHs may show a likely reduction in VTE recurrence, it may show an increase in major bleeding.

\section{Implications for research}

There is a need for research assessing patients' values and preferences regarding long-term anticoagulant agents for treating VTE. Researchers should consider making the raw data from randomized controlled trials (RCTs) available for individual participant data meta-analysis. Further RCTs including subgroups of people with cancer should report separate results for these subgroups.

\section{ACKNOWLEDGEMENTS}

We thank Dr Buller, Dr Deitcher, Dr Harenberg, Dr Huisman, Dr Hull, Dr Prandoni, Dr Reomera, Dr Schulman, Dr Belcaro and Dr Siragusa for their assistance. We thank Ms Ann Grifasi for her administrative support. We thank Dr Paola Muti, Dr Ignacio Neumann and Dr Nawman Labedi for their contributions to previous versions of this systematic review.
We thank Dr Assem Khamis for his help with conducting the sensitivity analysis.

We thank Jo Morrison, Co-ordinating Editor for the Cochrane Gynaecological, Neuro-oncology and Orphan Cancers Group. We also thank Gail Quinn, Managing Editor of the Cochrane Gynaecological Neuro-oncology and Orphan Cancers Group for her exceptional support. We thank Joanne Platt, the Information Specialist of the Cochrane Gynaecological Neuro-oncology and Orphan Cancers Group, for setting up and managing the monthly alerts.

As described under "Sources of Support" this update was supported in part by the American Society of Hematology to inform ASH guidelines on the topic. We thank the ASH guideline panel for prioritizing questions previously addressed by our review and for critically reviewing our work, including Drs. Pablo Alonso, Waleed Alhazanni, Marc Carrier, Cihan Ay, Marcello DiNisio, Lisa Hicks, Alok Khorana, Andrew Leavitt, Agnes Lee, Gary Lyman, Fergus Macbeth, Rebecca Morgan, Simon Noble, and David Stenehjem and patient representatives Jackie Cook and Elizabeth Sexton. Their input was valuable in validating some of the review related decisions such as eligibility of included studies and the analytical approach.

For our update of these reviews, we followed Cochrane methods using the same eligibility criteria and outcomes used previously. The ASH guidelines group used slightly different methods that generated slightly different results. For example, the ASH guideline panel agreed to prioritize different outcomes; include unpublished data; include abstracts; use different definitions for duration of treatment; and rate certainty of evidence slightly differently for some outcomes, for instance because of imprecision or indirectness. These differences are not described in this publication. Instead, they will be described in the ASH guideline publication.

This project was supported by the National Institute for Health Research (NIHR), via Cochrane Review Incentive Scheme Funding. The views and opinions expressed therein are those of the authors and do not necessarily reflect those of the Systematic Reviews Programme, NIHR, National Health Service or the Department of Health. 


\section{R E F E R E N C E S}

\section{References to studies included in this review}

Agnelli 2015 (AMPLIFY) \{published data only\}

Agnelli G, Buller H, Cohen A, Curto M, Gallus A, Johnson M, et al. Oral apixaban for the treatment of acute venous thromboembolism. New England Journal of Medicine 2013;369(9):799-808.

Agnelli G, Buller HR, Cohen A, Gallus AS, Lee TC, Pak R, et al. Apixaban for the treatment of venous thromboembolism in cancer patients: data from the amplify trial. Canadian Journal of Cardiology 2014;30:S278.

* Agnelli G, Buller HR, Cohen A, Gallus AS, Lee TC, Pak R, et al. Oral apixaban for the treatment of venous thromboembolism in cancer patients: results from the AMPLIFY trial. Journal of Thrombosis and Haemostasis 2015;13(12):2187-91.

\section{Agnelli 2020 (Caravaggio) \{published data only\}}

Ageno W, Vedovati MC, Cohen A, Huisman M, Bauersachs R, Gussoni G, Becattini C, Agnelli G. Bleeding with Apixaban and Dalteparin in Patients with Cancer-Associated Venous Thromboembolism: Results from the Caravaggio Study. Thrombosis and haemostasis 2020;Nov 17:1-9.

Agnelli G, Becattini C, Bauersachs R, Brenner B, Campanini M, Cohen A, Connors JM, Fontanella A, Gussoni G, Huisman MV, Lambert C. Apixaban versus dalteparin for the treatment of acute venous thromboembolism in patients with cancer: the Caravaggio study. Thrombosis and haemostasis 2018 Sep;118(09):1668-78.

* Agnelli G, Becattini C, Meyer G, Muñoz A, Huisman MV, Connors JM, Cohen A, Bauersachs R, Brenner B, Torbicki A, Sueiro MR. Apixaban for the treatment of venous thromboembolism associated with cancer. New England Journal of Medicine 2020 Apr 23;382(17):1599-607.

\section{Cesarone 2003 \{published data only\}}

Cesarone MR, Ledda A, Nicolaides A, Belcaro G, Geroulakos G. Three-month, outpatient, oral anticoagulant treatment in comparison with low-molecular-weight heparin in cancer patients. Circulation 2003;108(17):2875.

\section{Deitcher 2006 (ONCENOX) \{published data only\}}

Deitcher SR, Kessler CM, Lyons RM, Fareed J. Treatment of venous thromboembolic events (VTE) in patients with active malignancy: a randomized study of enoxaparin alone versus initial enoxaparin followed by warfarin for a 180-day period. Blood 2003;102(11):322A.

Deitcher SR, Kessler CM, Merli G, Rigas J, Lyons RM, Cort S. Secondary prevention of venous thromboembolic events (VTE) in patients with active malignancy: a randomized study of enoxaparin sodium alone versus initial enoxaparin sodium followed by warfarin for a 180-day period. Proceedings, annual meeting of the American Society of Clinical Oncology 22, 761Abstract no: 3060 2003;1(1):OC194.

* Deitcher SR, Kessler CM, Merli G, Rigas JR, Lyons RM, Fareed J, ONCENOX Investigators. Secondary prevention of venous thromboembolic events in patients with active cancer: enoxaparin alone versus initial enoxaparin followed by warfarin for a 180-day period. Clinical and Applied Thrombosis/ hemostasis 2006;12(4):389-96.

El Mokadem 2020 \{published data only\}

Mokadem ME, Hassan A, Algaby AZ. Efficacy and safety of apixaban in patients with active malignancy and acute deep venous thrombosis. Vascular 2020;Nov 5:1708538120971148.

\section{Hull 2006 (LITE) \{published data only\}}

Hull R, Pineo GF, Mah AF, Brant RF. A randomized trial evaluating long-term low-molecular-weight heparin therapy for three months vs. intravenous heparin followed by warfarin sodium in three patients with current cancer. Journal of Thrombosis and Haemostasis 2003;1:Abstract no: P1373a.

* Hull RD, Pineo GF, Brant RF, Mah AF, Burke N, Dear R, et al. Long-term low-molecular-weight heparin versus usual care in proximal-vein thrombosis patients with cancer. American Journal of Medicine 2006;119(12):1062-72.

Hull RD, Pineo GF, Brant RF. A randomized trial of the effect of low molecular weight heparin vs. warfarin on mortality in the long-term treatment of proximal vein thrombosis. Intensivmedizin und Notfallmedizin 2000;37(1 Suppl 1):123-32.

Hull RD, Pineo GF, Brant RF. Effect of low molecular weight heparin versus warfarin sodium on mortality in long-term treatment of proximal vein thrombosis. Clinical and Applied Thrombosis-Hemostasis 1996;2:S4-11.

\section{Lee 2003 (CLOT) \{published data only\}}

* Lee A, Levine M, Baker R, Bowden C, Kakkar AK, Prins M, et al. Low-molecular-weight heparin versus a coumarin for the prevention of recurrent venous thromboembolism in patients with cancer. New England Journal of Medicine 2003;349(2):146-53.

Lee AY, Julian JA, Levine MN, Baker RI, Bowden C, Kakkar AK, et al. Impact of dalteparin low-molecular-weight heparin (LMWH) on survival: results of a randomised trial in cancer patients with venous thromboembolism (VTE). Proceedings, annual meeting of the American Society of Clinical Oncology 2003;22:211-Abstract no: 846 .

Lee AY, Levine MN, Baker RI, Bowden C, Kakkar AK, Prins M, et al. Long-term treatment with dalteparin low-molecular-weight heparin (LMWH) is more effective than oral anticoagulant (OA) therapy in cancer patients with venous thromboembolism (VTE). Journal of Thrombosis and Haemostasis 2003;1:Abstract no: OC398.

Lee AY, Parpia S, Julian J, Rickles F, Prins M, Levine, M. Risk factors for recurrent thrombosis and anticoagulant-related bleeding in cancer patients. Journal of Thrombosis and Haemostasis 2009;7(S2):107.

Lee AY, Rickles FR, Julian JA, Gent M, Baker RI, Bowden C, et al. Randomized comparison of low molecular weight heparin and coumarin derivatives on the survival of patients with cancer 
and venous thromboembolism. Journal of Clinical Oncology 2005;23(10):2123-9.

Levine MN, Lee AY, Baker RI, Bowden C, Kakkar AK, Prins M, et al. A randomized trial of long term dalteparin low molecular weight heparin (LMWH) versus oral anticoagulant (OA) therapy in cancer patients with venous thromboembolism. 2002 Blood;100:Abstract no: 298.

Woodruff S, Feugere G, Abreu P, Heissler J, Jen F. A post-HOC analysis of dalteparin versus oral anticoagulant (VKA) therapy for the prevention of recurrent venous thromboembolism ( $\mathrm{rVTE}$ ) in patients with cancer and renal impairment. Journal of Thrombosis and Thrombolysis 2015;39(3):418.

\section{Lee 2015 (CATCH) \{published data only\}}

Bauersachs R, Lee AY, Kamphuisen PW, Meyer G, Janas MS, Jarner MF, et al, CATCH Investigators. Renal impairment, recurrent venous thromboembolism and bleeding in cancer patients with acute venous thromboembolism - analysis of the CATCH study. Thrombosis and Haemostasis 2018;118(5):914-21. [DOI: 10.1055/s-0038-1641150]

Bauersachs R, Lee AY, Kamphuisen PW, Meyer G, Janas MS, Jarner MF, et al. Long-term tinzaparin versus warfarin for treatment of venous thromboembolism (VTE) in cancer patients - analysis of renal impairment (RI) in the catch study. Journal of Thrombosis and Haemostasis 2015;13:76.

Bauersachs R. CATCH: a randomised clinical trial comparing long-term tinzaparin versus warfarin for treatment of acute venous thromboembolism in cancer patients. Hematology Reports 2011;3:Suppl 1.

Kamphuisen PW, Lee AY, Meyer G, Bauersachs R, Janas MS, Jarner MF, et al, CATCH Investigators. Clinically relevant bleeding in cancer patients treated for venous thromboembolism from the CATCH study. Journal of Thrombosis and Haemostasis 2018;16:1-9. [DOI: 10.1111/ jth.14007]

Kamphuisen PW, Lee AY, Meyer G, Bauersachs R, Janas MS, Jarner MF, et al. Characteristics and risk factors of major and clinically relevant non-major bleeding in cancer patients receiving anticoagulant treatment for acute venous thromboembolism - the CATCH study. Journal of Thrombosis and Haemostasis 2015;13:182-3.

Khorana AA, Bauersachs R, Kamphuisen PW, Meyer G, Janas MS, Jarner MF, et al. Clinical predictors of recurrent venous thromboembolism (VTE) in cancer patients from a randomized trial of long-term tinzaparin versus warfarin for treatment: the CATCH study. Journal of Clinical Oncology Conference 2015;33:15 Suppl 1.

Lee AY, Bauersachs R, Janas MS, Jarner MF, Kamphuisen PW, Meyer G, et al. CATCH: a randomised clinical trial comparing long-term tinzaparin versus warfarin for treatment of acute venous thromboembolism in cancer patients. BMC Cancer 2013;13(1):284. [NCT01130025]

Lee AY, Bauersachs R, Janas MS, Jarner MF, Kamphuisen PW, Meyer G, et al. CATCH: a randomized trial comparing tinzaparin versus warfarin for treatment of acute venous thromboembolism (VTE) in cancer patients. ASCO Annual Meeting. Journal of Clinical Oncology 2012;Suppl:TPS9149.

Lee AY, Kamphuisen PW, Meyer G, Bauersachs R, Janas MS, Jarner MF, et al. A randomized trial of long-term tinzaparin, a low molecular weight heparin (LMWH), versus warfarin for treatment of acute venous thromboembolism (VTE) in cancer patients - the CATCH study. Blood Conference: 56th Annual Meeting of the American Society of Hematology 2014;124:21.

* Lee AY, Kamphuisen PW, Meyer G, Bauersachs R, Janas MS, Jarner MF. Tinzaparin vs warfarin for treatment of acute venous thromboembolism in patients with active cancer: a randomized clinical trial. JAMA 2015;314:677.

\section{Lopez-Beret 2001 \{published data only\}}

Lopez-Beret P, Orgaz A, Fontcuberta J, Doblas M, Martinez A, Lozano $G$, et al. Low molecular weight heparin versus oral anticoagulants in the long-term treatment of deep venous thrombosis. Journal of Vascular Surgery 2001;33(1):77-90.

\section{Mazilu 2014 (OVIDIUS) \{published data only\}}

Mazilu L, Parepa IR, Suceveanu AI, Suceveanu A, Baz R, Catrinoiu D. Venous thromboembolism: secondary prevention with dabigatran vs. acenocumarol in patients with paraneoplastic deep vein thrombosis. Results from a small prospective study in Romania. Cardiovascular Research 2014;103(Suppl 1):S39, P221.

\section{McBane 2019 (ADAM-VTE) \{published data only\}}

McBane RD, Wysokinski WE, Le-Rademacher JG, Zemla T, Ashrani A, Tafur A, Perepu U, Anderson D, Gundabolu K, Kuzma C, Perez Botero J. Apixaban and dalteparin in active malignancy-associated venous thromboembolism: The ADAM VTE trial. Journal of thrombosis and haemostasis 2020 Feb;18(2):411-21.

\section{Meyer 2002 (CANTHANOX) \{published data only\}}

Gruel Y, Meyer G, Marjanovic Z, Valcke J, Lorcerie B, SolalCeligny $P$, et al. Canthanox, a randomized controlled study comparing low molecular weight heparin and warfarin for the prevention of recurrent venous thromboembolism in patients with cancer. Blood 2001;98:Abstract no: 2956.

\footnotetext{
* Meyer G, Marjanovic Z, Valcke J, Lorcerie B, Gruel Y, Solal-Celigny $P$, et al. Comparison of low-molecularweight heparin and warfarin for the secondary prevention of venous thromboembolism in patients with cancer: a randomized controlled study. Archives of Internal Medicine 2002;162(15):1729-35.
}

\section{Prins 2014 (EINSTEIN) \{published data only\}}

Einstein Investigators. Oral rivaroxaban for symptomatic venous thromboembolism. New England Journal of Medicine 2010;363:2499-510.

Einstein-PE Investigators. Oral rivaroxaban for the treatment of symptomatic pulmonary embolism. New England Journal of Medicine 2012;366:1287-97.

* Prins MH, Lensing AW, Brighton TA, Lyons RM, Rehm J, Trajanovic M, et al. Oral rivaroxaban versus enoxaparin with 
vitamin $\mathrm{K}$ antagonist for the treatment of symptomatic venous thromboembolism in patients with cancer (EINSTEIN-DVT and EINSTEIN-PE): a pooled subgroup analysis of two randomised controlled trials. Lancet Haematology 2014;1(1):e37-46.

\section{Raskob 2016 (HOKUSAI) \{published data only\}}

Hokusai-VTE Investigators. Edoxaban versus warfarin for the treatment of symptomatic venous thromboembolism. New England Journal of Medicine 2013;369:1406-15.

Raskob GE, Büller H, Prins M, Segers A, Shi M, Schwocho L, et al. Edoxaban for the long-term treatment of venous thromboembolism: rationale and design of the Hokusaivenous thromboembolism study - methodological implications for clinical trials. Journal of Thrombosis and Haemostasis 2013;11(7):1287-94

* Raskob GE, van Es N, Segers A, Angchaisuksiri P, Oh D, Boda Z, et al. Edoxaban for venous thromboembolism in patients with cancer: results from a non-inferiority subgroup analysis of the Hokusai-VTE randomised, double-blind, double-dummy trial. Lancet Haematology 2016;3(8):e379-87.

\section{Raskob 2018 (HOKUSAI) \{published data only\}}

* Raskob GE, van Es N, Verhamme P, Carrier M, Di Nisio M, Garcia D, et al. Edoxaban for the treatment of cancer-associated venous thromboembolism. New England Journal of Medicine 2018;378(7):615-24

Raskob GE, Van Es N, Verhamme P, Carrier M, Di Nisio M, Garcia DA, et al. A randomized, open-label, blinded outcome assessment trial evaluating the efficacy and safety of LMWH/ edoxaban versus dalteparin for venous thromboembolism associated with cancer: Hokusai VTE-Cancer Study (LBA-6). In: Blood. 2017.

\section{Romera 2009 \{published data only\}}

* Romera A, Cairols MA, Vila-Coll R, Martí X, Colomé E, Bonell A, et al. A randomised open-label trial comparing long-term subcutaneous low-molecular-weight heparin compared with oral-anticoagulant therapy in the treatment of deep venous thrombosis. European Journal of Vascular and Endovascular Surgery 2009;37(3):349-56.

Romera-Villegas A, Marti Mestre X, Vila Coll R, Colome Nafria E. Long-term treatment with a low-molecular-weight heparin administered subcutaneously compared with a vitamin $\mathrm{K}$ antagonist: subanalysis of patients with cancer. Medicina Clinica 2015;144:16-20.

\section{Schulman 2015 (RECOVER I-II) \{published data only\}}

* Schulman S, Goldhaber SZ, Kearon C, Kakkar AK, Schellong S, Eriksson $\mathrm{H}$. Treatment with dabigatran or warfarin in patients with venous thromboembolism and cancer. Thrombosis and Haemostasis 2015;114(1):150-7.

Schulman S, Kakkar AK, Goldhaber SZ, Schellong S, Eriksson H, Mismetti P, et al. Treatment of acute venous thromboembolism with dabigatran or warfarin and pooled analysis. Circulation 2014;129(7):764-72.

Schulman S, Kearon C, Kakkar AK, Mismetti P, Schellong S, Eriksson $\mathrm{H}$, et al. Dabigatran versus warfarin in the treatment of acute venous thromboembolism. New England Journal of Medicine 2009;361(24):2342-52.

van Doormaal 2010 (Van Gogh DVT trial) \{published data only\}

van Doormaal FF, Cohen AT, Davidson BL, Decousus H, Gallus AS, Gent M, et al. Idraparinux versus standard therapy in the treatment of deep venous thrombosis in cancer patients: a subgroup analysis of the Van Gogh DVT trial. Thrombosis and Haemostasis 2010;104(1):86-91.

\section{Young 2018 (SELECT-D) \{published data only\}}

Young A, Dunn J, Chapman O, Grumett J, Marshall A, Phillips J, et al. SELECT-D: anticoagulation therapy in selected cancer patients at risk of recurrence of venous thromboembolism. ASCO Annual Meeting Proceedings 2014;32(15 Suppl):TPS9661.

Young A, Marshall A, Thirlwall J, Hill C, Hale D, Dunn J, et al. Anticoagulation therapy in selected cancer patients at risk of recurrence of venous thromboembolism: results of the Select$\mathrm{D}^{\mathrm{TM}}$ pilot trial. Blood 2017;130:625.

Young A, Phillips J, Hancocks H, Hill C, Joshi N, Marshall A, et al. OC-11 - anticoagulation therapy in selected cancer patients at risk of recurrence of venous thromboembolism. Thrombosis Research 2016;140:S172-3.

Young A, Phillips J, Hancocks H, Marshall A, Grumett J, Dunn J, et al. Anticoagulation therapy in selected cancer patients at risk of recurrence of venous thromboembolism. ASCO Annual Meeting Proceedings 2015;33(15 Suppl):TPS9642.

* Young AM, Marshall A, Thirlwall J, Chapman O, Lokare A, Hill C, Hale D, Dunn JA, Lyman GH, Hutchinson C, MacCallum P. Comparison of an oral factor Xa inhibitor with low molecular weight heparin in patients with cancer with venous thromboembolism: results of a randomized trial (SELECT-D). $J$ Clin Oncol 2018 Jul 10 ;36(20):2017-2023.

\section{References to studies excluded from this review}

\section{Agnelli 2005 \{published data only\}}

Agnelli G, Bergqvist D, Cohen AT, Gallus AS, Gent M. Randomized clinical trial of postoperative fondaparinux versus perioperative dalteparin for prevention of venous thromboembolism in high-risk abdominal surgery. British Journal of Surgery 2005;92(10):1212-20.

\section{Alikhan 2003 (MEDENOX) \{published data only\}}

* Alikhan R, Cohen AT, Combe S, Samama MM, Desjardins L, Eldor A, et al. Prevention of venous thromboembolism in medical patients with enoxaparin: a subgroup analysis of the MEDENOX study. Blood Coagulation \& Fibrinolysis 2003;14(4):341-6.

Samama MM, Cohen AT, Darmon JY, Desjardins L, Eldor A, Janbon C, et al. A comparison of enoxaparin with placebo for the prevention of venous thromboembolism in acutely ill medical patients. New England Journal of Medicine 1999;341(11):793-800. 
Auer 2011 \{published data only\}

Auer R, Scheer A, Wells PS, Boushey R, Asmis T, Jonker D, et al. The use of extended perioperative low molecular weight heparin (tinzaparin) to improve disease-free survival following surgical resection of colon cancer: a pilot randomized controlled trial. Blood Coagulation \& Fibrinolysis 2011;22(8):760-2.

\section{Cohen 2006 \{published data only\}}

Cohen AT, Davidson BL, Gallus AS, Lassen MR, Prins MR, Tomkowski W, et al. Efficacy and safety of fondaparinux for the prevention of venous thromboembolism in older acute medical patients: randomised placebo controlled trial. $B M J$ 2006;332(7537):325-9.

\section{Cohen 2007 (PREVENT) \{published data only\}}

Cohen AT, Davidson BL, Gallus AS, Lassen MR, Prins MH, Tomkowski W, et al. Efficacy and safety of fondaparinux for the prevention of venous thromboembolism in older acute medical patients: randomised placebo controlled trial. BMJ 2006;332(7537):325-9.

* Cohen AT, Turpie AG, Leizorovicz A, Olsson CG, Vaitkus PT, Goldhaber SZ. Thromboprophylaxis with dalteparin in medical patients: which patients benefit? Vascular Medicine 2007;12(2):123-7.

Leizorovicz A, Cohen AT, Turpie AG, Olsson CG, Vaitkus PT, Goldhaber SZ, PREVENT Medical Thromboprophylaxis Study Group. Randomized, placebo-controlled trial of dalteparin for the prevention of venous thromboembolism in acutely ill medical patients. Circulation 2004;110(7):874-9.

\section{Couban 2005 \{published data only\}}

Couban S, Goodyear M, Burnell M, Dolan S, Wasi P, Barnes D, et al. A randomized double-blind placebo-controlled study of low dose warfarin for the prevention of symptomatic central venous catheter-associated thrombosis in patients with cancer. In: Blood, American Society of Hematology 44th Annual Meeting. 2002:Abstract no 2769.

Couban S, Goodyear M, Burnell M, Dolan S, Wasi P, Barnes D, et al. A randomized double-blind placebo controlled study of low dose warfarin for the prevention of symptomatic central venous catheter-associated thrombosis in patients with cancer. Journal of Thrombosis and Haemostasis 2003;1:Abstract no: P198.

* Couban S, Goodyear M, Burnell M, Dolan S, Wasi P, Barnes D, et al. Randomized placebo-controlled study of low-dose warfarin for the prevention of central venous catheterassociated thrombosis in patients with cancer. Journal of Clinical Oncology 2005;23(18):4063-9.

\section{Eriksson 2005 \{published data only\}}

Eriksson H, Lundstrom T, Wahlander K, Clason SB, Schulman S. Prognostic factors for recurrence of venous thromboembolism (VTE) or bleeding during long-term secondary prevention of VTE with ximelagatran. Thrombosis and Haemostasis 2005;94(3):522-7.

\section{Farred 2004 \{published data only\}}

Farred J. Multifactorial etiology of cancer associated venous thrombosis: results from profiling of cancer patients recruited in a study of the secondary prevention of thrombosis with low molecular weight heparin. In: 40th Annual Meeting for the American Society for Clinical Oncology; 2004 Jun 5-8; New Orleans (LA). 2004.

\section{Ferretti 2005 \{published data only\}}

Ferretti G, Bria E, Carlini P, Felici A, Giannarelli D, Ciccarese M, et al. Meta-analysis of the randomized comparisons between low-molecular weight heparin (LMWH) with oral anticoagulants (OA) for the long-term treatment of symptomatic venous thromboembolism (VTE): no difference in cancer-related mortality. Journal of Clinical Oncology 2005;23(16):765S.

\section{Ferretti 2006 \{published data only\}}

Ferretti G. Does low-molecular-weight heparin influence cancerrelated mortality? Annals of Oncology 2006;17(10):1604-6.

\section{Fiessinger 2005 \{published data only\}}

Fiessinger JN, Huisman MV, Davidson BL, Bounameaux $\mathrm{H}$, Francis $\mathrm{CW}$, Eriksson $\mathrm{H}$, et al. Ximelagatran vs low-molecularweight heparin and warfarin for the treatment of deep vein thrombosis - a randomized trial. JAMA 2005;293(6):681-9.

\section{Haas 2011 \{published data only\}}

Bauersachs R, Schellong SM, Haas S, Tebbe U, Gerlach HE, Abletshauser C, et al. CERTIFY: prophylaxis of venous thromboembolism in patients with severe renal insufficiency. Thrombosis and Haemostasis 2011;105(6):981-8.

Haas S, Schellong SM, Tebbe U, Gerlach HE, Bauersachs R, Abletshauser C, et al. CERTIFY: certoparin versus UFH to prevent venous thromboembolic events in the patients with cancer. Hämostaseologie 2011;31(1):A10.

* Haas S, Schellong SM, Tebbe U, Gerlach HE, Bauersachs R, Melzer N, et al. Heparin based prophylaxis to prevent venous thromboembolic events and death in patients with cancer - a subgroup analysis of CERTIFY. BMC Cancer 2011;11(1):1.

\section{Hata 2016 \{published data only\}}

Hata K, Kimura T, Tsuzuki S, Ishii G, Kido M, Yamamoto T, et al. Safety of fondaparinux for prevention of postoperative venous thromboembolism in urological malignancy: a prospective randomized clinical trial. International Journal of Urology 2016;23(11):923-8.

\section{Hull 2007 \{published data only\}}

Hull RD, Pineo GF, Brant RF, Mah AF, Burke N, Dear R, et al. Selfmanaged long-term low-molecular-weight heparin therapy: the balance of benefits and harms. American Journal of Medicine 2007;120:72-82.

\section{Hull 2009 \{published data only\}}

Hull RD, Pineo GF, Brant R, Liang J, Cook R, Solymoss S, et al. Home therapy of venous thrombosis with long-term LMWH versus usual care: patient satisfaction and post-thrombotic syndrome. American Journal of Medicine 2009;122:762-9. 


\section{Hyers 2005 \{published data only\}}

Hyers TM. Long-term anticoagulation prophylaxis following acute thromboembolism. Disease-a-Month 2005;51(2-3):158-65.

Kakkar 2003 \{published data only\}

Kakkar VV, Gebska M, Kadziola Z, Saba N, Carrasco P. Lowmolecular-weight heparin in the acute and long-term treatment of deep vein thrombosis. Thrombosis and Haemostasis 2003;89(4):674-80.

\section{Kakkar 2010 (CANBESURE) \{published data only\}}

Kakkar VV, Balibrea J, Martinez-Gonzalez J, Prandoni P. Late breaking clinical trial: a randomised double blind trial to evaluate the efficacy and safety of prolonging the thromboprophylaxis with bemiparin in patients undergoing cancer abdominal or pelvic surgery (the CANBESURE study). International Society on Thrombosis and Haemostasis 2009;5(6):1223-9.

* Kakkar VV, Balibrea JL, Martinez-Gonzalez J, Prandoni P. Extended prophylaxis with bemiparin for the prevention of venous thromboembolism after abdominal or pelvic surgery for cancer: the CANBESURE randomized study. Journal of Thrombosis and Haemostasis : JTH 2010;8(6):1223-9.

Kakkar 2014 (SAVE-ABDO) \{published data only\}

* Kakkar AK, Agnelli G, Fisher WD, George D, Lassen MR, Mismetti P, et al, SAVE-ABDO Investigators. Preoperative enoxaparin versus postoperative semuloparin thromboprophylaxis in major abdominal surgery: a randomized controlled trial. Annals of Surgery 2014;259(6):1073-9.

Kakkar AK, Agnelli G, Fisher WD, George D, Mouret P, Lassen MR, et al. The ultra-low-molecular-weight heparin semuloparin for prevention of venous thromboembolism In patients undergoing major abdominal surgery. Blood 2010;116(21):188.

\section{Khorana 2017 (PHACS) \{published data only\}}

Khorana AA, Francis CW, Kuderer N, Carrier M, Ortel TL, Wun T, et al. Dalteparin thromboprophylaxis in cancer patients at high risk for venous thromboembolism: a randomized trial. Blood 2015;126(23):427.

* Khorana AA, Francis CW, Kuderer N, Carrier M, Ortel TL, Wun T, et al. Dalteparin thromboprophylaxis in cancer patients at high risk for venous thromboembolism: a randomized trial. Thrombosis Research 2017;151:89-95. [DOI: dx.doi.org/10.1016/ j.thromres.2017.01.009]

\section{King 2005 \{published data only\}}

King KM, Wong C, Nutescu E, Shord SS. Warfarin dose requirements in cancer and non-cancer. Pharmacotherapy 2005;25(3):468

\section{Kovacs 2005 \{published data only\}}

Kovacs MJ, Levine MN, Keeney M, Mackinnon KM, Lee AY. AntiXa effect of a low molecular weight heparin (dalteparin) does not accumulate in extended duration therapy for venous thromboembolism in cancer patients. Thrombosis and Haemostasis 2005;93(6):1185-8.

\section{Kucher 2005 \{published data only\}}

Kucher N, Quiroz R, McKean S, Sasahara AA, Goldhaber SZ, Kucher N, et al. Extended enoxaparin monotherapy for acute symptomatic pulmonary embolism. Vascular Medicine 2005;10(4):251-6

\section{Larocca 2012 \{published data only\}}

Larocca A, Cavallo F, Bringhen S, Raimondo FD, Falanga A, Evangelista A, et al. Aspirin or enoxaparin thromboprophylaxis for patients with newly diagnosed multiple myeloma treated with lenalidomide. Blood 2012;119:933-9.

Lee 2005 \{published data only\}

Lee A, Levine M. Treatment of venous thromboembolism in cancer patients. Cancer Control 2005;12 Suppl 1:17-21.

Lee 2006 \{published data only\}

Lee AY. Dalteparin sodium in the management of thromboembolic disorder. Therapy 2006;3(4):461-73.

Levine 2003 \{published data only\}

Levine MN, Lee AY, Kakkar AK. From Trousseau to targeted therapy: new insights and innovations in thrombosis and cancer. Thrombosis and Haemostasis 2003;1(7):1456-63.

\section{Macbeth 2016 (FRAGMATIC) \{published data only\}}

Griffiths GO, Burns S, Noble SI, Macbeth FR, Cohen D, Maughan TS. FRAGMATIC: a randomised phase III clinical trial investigating the effect of Fragmin ${ }^{\circledR}$ added to standard therapy in patients with lung cancer. BMC Cancer 2009;9(1):1.

* Macbeth F, Noble S, Evans J, Ahmed S, Cohen D, Hood K, et al. Randomized phase III trial of standard therapy plus low molecular weight heparin in patients with lung cancer: FRAGMATIC trial. Journal of Clinical Oncology 2016;34(5):488-94.

Macbeth F, Noble S, Griffiths G, Chowdhury R, Rolfe C, Hood K, et al. Preliminary results from the FRAGMATIC trial: a randomised phase iii clinical trial investigating the effect of Fragmin $(\mathrm{R})$ added to standard therapy in patients with lung cancer. Journal of Thoracic Oncology 2013;8:S243.

Noble S, Robbins A, Alikhan R, Hood K, Macbeth F. Prediction of venous thromboembolism in lung cancer patients receiving chemotherapy. Journal of Thrombosis and Haemostasis 2015;13:143.

\section{Massicotte 2003 \{published data only\}}

Massicotte P, Julian JA, Gent M, Shields K, Marzinotto V, Szechtman B, et al. An open-label randomized controlled trial of low molecular weight heparin compared to heparin and coumadin for the treatment of venous thromboembolic events in children: the REVIVE trial. Thrombosis Research 2003;109(2-3):85-92.

\section{Murakami 2002 \{published data only\}}

Murakami M, Wiley LA, Cindrick-Pounds L, Hunter GC, Uchida T, Killewich LA. External pneumatic compression does not increase urokinase plasminogen activator after abdominal surgery. Vascular Surgery 2002;36(5):917-21. 
Nagata 2015 \{published data only\}

Nagata C, Tanabe H, Takakura S, Narui C, Saito M, Yanaihara N, et al. Randomized controlled trial of enoxaparin versus intermittent pneumatic compression for venous thromboembolism prevention in Japanese surgical patients with gynecologic malignancy. Journal of Obstetrics and Gynaecology Research 2015;41(9):1440-8.

\section{Palumbo 2011 \{published data only\}}

Cavo M, Palumbo A, Bringhen S, Di Raimondo F, Patriarca F, Rossi $D$, et al. Phase III study of enoxaparin versus aspirin versus low-dose warfarin as thromboprophylaxis for patients with newly diagnosed multiple myeloma treated upfront with thalidomide-containing regimens. Haematologica 2010;95:391.

Cavo M, Palumbo A, Bringhen S, Falcone A, Musto P, Ciceri F, et al. A phase III study of enoxaparin versus low-dose warfarin versus aspirin as thromboprophylaxis for patients with newly diagnosed multiple myeloma treated up-front with thalidomide-containing regimens. Blood 2008;112(11):3017.

Cavo M, Palumbo A, Bringhen S, Falcone A, Musto P, Ciceri F, et al. A phase III study of enoxaparin versus low-dose warfarin versus aspirin as thromboprophylaxis for patients with newly diagnosed multiple myeloma treated up-front with thalidomide-containing regimens. Haematologica 2009;94:s4.

Magarotto V, Brioli A, Patriarca F, Rossi D, Petrucci MT, Nozzoli C, et al. Enoxaparin, aspirin, or warfarin for the thromboprophylaxis in newly diagnosed myeloma patients receiving thalidomide: a randomized controlled trial. XI Congress of the Italian Society of Experimental Hematology 2010;95:S1-S162.

Palumbo A, Cavo M, Bringhen S, Zaccaria A, Spadano A, Palmieri $S$, et al. Enoxaparin versus aspirin versus low-fixeddose of warfarin in newly diagnosed myeloma patients treated with thalidomide-containing regimens: a randomized, controlled trial. Haematologica 2008;93:362.

* Palumbo A, Cavo M, Bringhen S, Zamagni E, Romano A, Patriarca F, et al. Aspirin, warfarin, or enoxaparin thromboprophylaxis in patients with multiple myeloma treated with thalidomide: a phase III, open-label, randomized trial. Journal of Clinical Oncology 2011;29:986-93.

\section{Pelzer 2015 (CONKO-004) \{published data only\}02140505}

Pelzer U, Deutschinoff G, Opitz B, Stauch M, Reitzig P, Hahnfeld $S$, et al. A prospective, randomized trial of simultaneous pancreatic cancer treatment with enoxaparin and chemotherapy - first results of the CONKO 004 trial. In: Onkologie - DGHO meeting. Vol. 580. October 2009:Abstract.

Pelzer U, Hilbig A, Stieler J, Roll L, Riess H, Dorken B, et al. A prospective, randomized trial of simultaneous pancreatic cancer treatment with enoxaparin and chemotherapy (PROSPECT - CONKO 004). Onkologie 2005;28(Suppl 3):54.

Pelzer U, Hilbig A, Stieler J, Roll L, Stauch M, Opitz B, et al. A prospective, randomized trial of simultaneous pancreatic cancer treatment with enoxaparin and chemotherapy (PROSPECT-CONKO 004). ASCO Annual Meeting Proceedings 2006;24(18):4110.
Pelzer U, Hilbig A, Stieler JM, Bahra M, Sinn M, Gebauer B, et al. Intensified chemotherapy and simultaneous treatment with heparin in outpatients with pancreatic cancer - the CONKO 004 pilot trial. BMC Cancer 2014;14:204.

Pelzer U, Oettle H, Stauch M, Opitz B, Stieler J, Scholten T, et al. Prospective, randomized open trial of enoxaparin in patients with advanced pancreatic cancer undergoing first-line chemotherapy. In: XXIst Congress of the International Society on Thrombosis and Haemostasis; 2007 Jul 6-12; Geneva. 2007:PT-488.

* Pelzer U, Opitz B, Deutschinoff G, Stauch M, Reitzig PC, Hahnfeld S, et al. Efficacy of prophylactic low-molecular weight heparin for ambulatory patients with advanced pancreatic cancer: outcomes from the CONKO-004 trial. Journal of Clinical Oncology 2015;3(18):2028-34.

Riess H, Pelzer U, Deutschinoff G, Opitz B, Stauch M, Reitzig P, et al. A prospective, randomized trial of chemotherapy with or without the low molecular weight heparin (LMWH) enoxaparin in patients (pts) with advanced pancreatic cancer (APC): results of the CONKO 004 trial. ASCO Annual Meeting Proceedings 2009;27(18S):LBA4506.

Riess H, Pelzer U, Hilbig A, Stieler J, Opitz B, Scholten T, et al. Rationale and design of PROSPECT-CONKO 004: a prospective, randomized trial of simultaneous pancreatic cancer treatment with enoxaparin and chemotherapy. BMC Cancer 2008;8:361.

Riess H, Pelzer U, Opitz B, Stauch M, Reitzig P, Hahnfeld S, et al. A prospective, randomized trial of simultaneous pancreatic cancer treatment with enoxaparin and chemotherapy: final results of the CONKO-004 trial. Journal of Clinical Oncology Conference 2010;28(15 Suppl):4033.

Riess HB, Pelzer U, Opitz B, Hilbig A, Strauch M, Hahnfeld S, et al. Late breaking clinical trial: a prospective, randomized trial of chemotherapy with and without the low molecular weight heparin (LMWH) enoxaparin in advanced pancreatic cancer patients. International Society on Thrombosis and Haemostasis 2009;7(Suppl 2):1203.

\section{Pérez-de-Llano 2010 \{published data only\}}

Pérez-de-Llano LA, Leiro-Fernández V, Golpe R, NúñezDelgado JM, Palacios-Bartolomé A, Méndez-Marote L, et al. Comparison of tinzaparin and acenocoumarol for the secondary prevention of venous thromboembolism: a multicentre, randomized study. Blood Coagulation Fibrinolysis 2010;21(8):744-99.

\section{Sakon 2010 \{published data only\}}

Sakon M, Kobayashi T, Shimazui T. Efficacy and safety of enoxaparin in Japanese patients undergoing curative abdominal or pelvic cancer surgery: results from a multicenter, randomized, open-label study. Thrombosis Research 2010;125(3):e65-70.

\section{Schulman 2003 \{published data only\}}

Schulman S, Wahlander K, Lundstrom T, Clason SB, Eriksson $\mathrm{H}$, Investigators $\mathrm{TI}$, et al. Secondary prevention of venous thromboembolism with the oral direct thrombin 
inhibitor ximelagatran. New England Journal of Medicine 2003;349(18):1713-21.

\section{Schulman 2006 \{published data only\}}

Schulman S, Lindmarker P, Holmström M, Lärfars G, Carlsson A, Nicol $P$, et al. Post-thrombotic syndrome, recurrence, and death 10 years after the first episode of venous thromboembolism treated with warfarin for 6 weeks or 6 months. Thrombosis \& Haemostasis 2006;4(4):734-42.

\section{Schulman 2013 (RE-MEDY) \{published data only\}}

Schulman S, Kearon C, Kakkar AK, Schellong S, Eriksson H, Baanstra D, et al. Extended use of dabigatran, warfarin, or placebo in venous thromboembolism. New England Journal of Medicine 2013;368(8):709-18.

\section{Siragusa 2010 \{published data only\}}

Siragusa S, Malato A, Mascheroni D, Ageno W, Bucherini E, Spadaro $P$, et al. The optimal duration of anticoagulant therapy in patients with cancer-related deep vein thrombosis: the advantage of using residual vein thrombosis (the Cancer-DACUS Study). Blood 2010;116(21):87-8.

\section{Song 2014 \{published data only\}}

Song KY, Yoo HM, Kim EY, Kim JI, Yim HW, Jeon HM, et al. Optimal prophylactic method of venous thromboembolism for gastrectomy in Korean patients: an interim analysis of prospective randomized trial. Annals of Surgical Oncology 2014;21(13):4232-8.

\section{Suarez Alvarez 2003 \{published data only\}}

Suarez Alvarez CG, Garcia Canete J, Herrero Mendoza MD, Bellver Alvarez TM, Arboiro Pinel R. Treatment of deep vein thrombosis with low molecular weight heparins at home. Anales de Medicina Interna 2003;20(3):134-6.

\section{Vedovati 2014 \{published data only\}}

Becattini C, Rondelli F, Vedovati MC, Camporese G, Giustozzi M, Boncompagni $\mathrm{M}$, et al. Incidence and risk factors for venous thromboembolism after laparoscopic surgery for colorectal cancer. Haematologica 2015;100(1):e35-8.

Becattini C, Vedovati MC, Rondelli F, Boncompagni M, Camporese G, Balzarotti R, et al. One week vs. four week heparin prophylaxis after laparoscopic surgery for colorectal cancer. The PRO-LAPS pilot feasibility study. In: International Society on Thrombosis and Haemostasis. 2013:ATT05, 1-105.

Vedovati MC, Becattini C, Rondelli F, Boncompagni M, Camporese G, Balzarotti R, et al. A randomized study on 1 vs. 4 weeks prophylaxis for venous thromboembolism after laparoscopic surgery for colorectal cancer. Thrombosis and Haemostasis 2013;11:214.

* Vedovati MC, Becattini C, Rondelli F, Boncompagni M, Camporese G, Balzarotti R, et al. A randomized study on 1-week versus 4-week prophylaxis for venous thromboembolism after laparoscopic surgery for colorectal cancer. Annals of Surgery 2014;259(4):665-9.

\section{Veiga 2000 \{published data only\}}

Veiga F, Escriba A, Maluenda MP, Lopez Rubio M, Margalet I, Lezana A, et al. Low molecular weight heparin (enoxaparin) versus oral anticoagulant therapy (acenocoumarol) in the long-term treatment of deep venous thrombosis in the elderly: a randomized trial. Thrombosis and Haemostasis 2000;84(4):559-64.

\section{Verso 2008 \{published data only\}}

Agnelli G, Verso M, Bertoglio S, Ageno W, Bazzan M, Parise P, et al. A double-blind placebo-controlled randomized study on the efficacy and safety of enoxaparin for the prevention of upper limb deep vein thrombosis in cancer patients with central vein catheter. Journal of Clinical Oncology 2004;22:734S.

Verso M, Agnelli G, Bertoglio S, Di Somma FC, Paoletti F, Ageno W, et al. Enoxaparin for the prevention of venous thromboembolism associated with central vein catheter: a double-blind, placebo-controlled, randomized study in cancer patients. Journal of Clinical Oncology 2005;23(18):4057-62.

Verso M, Agnelli G, Kamphuisen PW, Ageno W, Bazzan M, Lazzaro A, et al. A double-blind placebo-controlled randomized study on the efficacy and safety of enoxaparin for the prevention of upper limb deep vein thrombosis in cancer patients with central vein catheter. Thrombosis and Haemostasis 2003;1:Abstract no: P0825.

* Verso M, Agnelli G, Kamphuisen PW, Ageno W, Bazzan M, Lazzaro A, et al. Risk factors for upper limb deep vein thrombosis associated with the use of central vein catheter in cancer patients. Internal and Emergency Medicine 2008;3(2):117-22.

\section{Zheng 2014 \{published data only\}}

Zheng H, Gao Y, Yan X, Gao M, Gao W. Prophylactic use of low molecular weight heparin in combination with graduated compression stockings in post-operative patients with gynecologic cancer. Zhonghua Zhong Liu za Zhi [Chinese Journal of Oncology] 2014;36(1):39-42.

\section{Zwicker 2013 (MICROTEC) \{published data only\}}

Zwicker J, Liebman HA, Bauer KA, Caughey T, Rosovsky R, Mantha S, et al. A randomized-controlled phase II trial of primary thromboprophylaxis with enoxaparin in cancer patients with elevated tissue factor bearing microparticles (the MICROTEC study). Thrombosis and Haemostasis 2013;11:6.

* Zwicker JI, Liebman HA, Bauer KA, Caughey T, Campigotto F, Rosovsky R, et al. Prediction and prevention of thromboembolic events with enoxaparin in cancer patients with elevated tissue factor-bearing microparticles: a randomized-controlled phase II trial (the MICROTEC study). British Journal of Haematology 2013;160(4):530-7.

\section{References to ongoing studies}

\section{Kamphuisen 2010 (Longheva) \{published data only\}}

Kleinjan A, Di Nisio M, Kamphuisen PW, Buller HR, on behalf of the steering committee. Long-term treatment for cancer patients with deep vein thrombosis or pulmonary embolism 
- a randomized controlled trial. 5th ICTHIC Abstracts: Poster Sessions / Thrombosis Research 2010;125(PO-67):S184.

Karatas 2015 \{published data only\}

Rivaroxaban in the treatment of venous thromboembolism (VTE) in cancer patients. Ongoing study. March 2016. Contact author for more information.

\section{Meyer 2016 (CASTA-DIVA) \{published data only\}}

Cancer associated thrombosis, a pilot treatment study using rivaroxaban (CASTA-DIVA). Ongoing study. September 2016. Contact author for more information.

\section{Ryun Park 2017 (PRIORITY) \{published data only\}}

A randomized phase II study to compare the safety and efficacy of dalteparin vs. rivaroxaban for cancer-associated venous thromboembolism (PRIORITY). Ongoing study. May 2017. Contact author for more information.

\section{Schrag 2016 (CANVAS) \{published data only\}}

Direct oral anticoagulants (DOACs) versus $\mathrm{LMWH} \pm$ warfarin for VTE in cancer (CANVAS). Ongoing study. December 2016. Contact author for more information.

\section{Additional references}

\section{Agnelli 2013}

Agnelli G, Buller HR, Cohen A, Curto M, Gallus AS, Johnson M, et al. Oral apixaban for the treatment of acute venous thromboembolism. New England Journal of Medicine 2013;369(9):799-808.

\section{Akl 2013}

Akl EA, Johnston BC, Alonso-Coello P, Neumann I, Ebrahim S, Briel $\mathrm{M}$, et al. Addressing dichotomous data for participants excluded from trial analysis: a guide for systematic reviewers. PloS One 2013;8(2):e57132.

\section{Akl 2016}

Akl EA, Kahale LA, Ebrahim S, Alonso-Coello P, Schünemann HJ, Guyatt GH. Three challenges described for identifying participants with missing data in trials reports, and potential solutions suggested to systematic reviewers. Journal of Clinical Epidemiology 2016;76:147-54.

\section{Akl 2017}

Akl EA, Meerpohl JJ, Elliott JH, Kahale LA, Schünemann HJ on behalf of the Living Systematic Review Network. Living systematic reviews: 4 . living guideline recommendations. Journal of Clinical Epidemiology 2017;91:47-53.

\section{Alshurafa 2012}

Alshurafa M, Briel M, Akl EA, Haines T, Moayyedi P, Gentles SJ, et al. Inconsistent definitions for intention-to-treat in relation to missing outcome data: systematic review of the methods literature. PloS One 2012;7(11):e49163.

\section{Ansell 2008}

Ansell J, Hirsh J, Hylek E, Jacobson A, Crowther M, Palareti G. Pharmacology and management of the vitamin $\mathrm{K}$ antagonists:
American College of Chest Physicians evidence-based clinical practice guidelines (8th edition). Chest 2008;133(Suppl 6):160S-98S.

\section{Cochrane Crowd}

Cochrane Crowd. Cochrane Crowd. crowd.cochrane.org/ index.html (accessed 9 June 2018).

\section{Conti 2003}

Conti S, Guercini F, lorio A. Low-molecular-weight heparin and cancer survival: review of the literature and pooled analysis of 1,726 patients treated for at least three months. Pathophysiology of Haemostasis \& Thrombosis 2003;33(4):197-201.

\section{CSR-web}

Cochrane Community. CRS (Cochrane Register of Studies). community.cochrane.org/help/tools-and-software/crscochrane-register-studies (accessed 9 June 2018).

\section{Deeks 2001}

Deeks JJ, Altman DG, Bradburn MJ. Statistical methods for examining heterogeneity and combining results from several studies in meta-analysis. In: Egger M, Davey Smith G, Altman DG, editors(s). Systematic Reviews in Health Care: Meta-Analysis in Context. 2nd edition. London: BMJ Publication Group, 2001.

\section{DerSimonian 1986}

DerSimonian R, Laird N. Meta-analysis in clinical trials. Controlled Clinical Trials 1986;7:177-88.

\section{Ebrahim 2013}

Ebrahim S, Akl EA, Mustafa RA, Sun X, Walter SD, HeelsAnsdell $D$, et al. Addressing continuous data for participants excluded from trial analysis: a guide for systematic reviewers. Journal of Clinical Epidemiology 2013;66(9):1014-21.

\section{Gallus 1997}

Gallus AS. Prevention of post-operative deep leg vein thrombosis in patients with cancer. Thrombosis and Haemostasis 1997;78(1):126-32.

\section{GRADE Handbook}

Schünemann H, Brożek J, Guyatt G, Oxman A. GRADE Handbook. gdt.guidelinedevelopment.org/app/handbook/ handbook.html Updated October 2013.

\section{Guyatt 2017}

Guyatt GH, Ebrahim S, Alonso-Coello P, Johnston BC, Mathioudakis AG, Briel M, et al. GRADE guidelines 17: assessing the risk of bias associated with missing participant outcome data in a body of evidence. Journal of Clinical Epidemiology 2017;87:14-22.

\section{Haynes 2002}

Haynes RB, Devereaux PJ, Guyatt GH. Clinical expertise in the era of evidence-based medicine and patient choice. Vox Sanguinis 2002;83 Suppl 1:383-6. 


\section{Heit 2000}

Heit JA, Silverstein MD, Mohr DN, Petterson TM, O'Fallon WM, Melton LJ. Risk factors for deep vein thrombosis and pulmonary embolism: a population-based case-control study. Archives of Internal Medicine 2000;160(6):809-15.

\section{Higgins 2011}

Higgins JP, Green S, editor(s). Cochrane Handbook for Systematic Reviews of Interventions Version 5.1.0 (updated March 2011). The Cochrane Collaboration, 2011. Available from handbook.cochrane.org.

\section{Hirsh 1993}

Hirsh J. Low molecular weight heparin. Thrombosis and Haemostasis 1993;70(1):204-7.

\section{Hutten 2000}

Hutten BA, Prins MH, Gent M, Ginsberg J, Tijssen JG, Buller HR. Incidence of recurrent thromboembolic and bleeding complications among patients with venous thromboembolism in relation to both malignancy and achieved international normalized ratio: a retrospective analysis. Journal of Clinical Oncology 2000;18(17):3078-83.

\section{Kahale 2019}

Kahale LA, Guyatt GH, Agoritsas T, Briel M, Busse JW, CarrascoLabra A, Khamis AM, Zhang Y, Hooft L, Scholten RJ, Akl EA. A guidance was developed to identify participants with missing outcome data in randomized controlled trials. Journal of clinical epidemiology 2019;115:55-63.

\section{Kahale 2020}

Kahale LA, Khamis AM, Diab B, Chang Y, Lopes LC, Agarwal A, Li L, Mustafa RA, Koujanian S, Waziry R, Busse JW. Potential impact of missing outcome data on treatment effects in systematic reviews: imputation study.. BMJ 2020;370:m2898.

\section{Kakkar 1970}

Kakkar VV, Howe CT, Nicolaides AN, Renney JT, Clarke MB. Deep vein thrombosis of the leg. Is there a "high risk" group? American Journal of Surgery 1970;120(4):527-30.

\section{Laporte 2011}

Laporte S, Liotier J, Bertoletti L, Kleber FX, Pineo GF, Chapelle C, et al. Individual patient data meta-analysis of enoxaparin vs. unfractionated heparin for venous thromboembolism prevention in medical patients. Thrombosis and Haemostasis 2011;9(3):464-72.

\section{Levitan 1999}

Levitan N, Dowlati A, Remick SC, Tahsildar HI, Sivinski LD, Beyth R, et al. Rates of initial and recurrent thromboembolic disease among patients with malignancy versus those without malignancy: risk analysis using Medicare claims data. Medicine 1999;78(5):285-91.

\section{Noble 2008}

Noble SI, Shelley MD, Coles B, Williams SM, Wilcock A, Johnson MJ. Management of venous thromboembolism in patients with advanced cancer: a systematic review and metaanalysis. Lancet 2008;9(6):577-84.

\section{Parmar 1998}

Parmar MKB, Torri V, Stewart L. Extracting summary statistics to perform meta-analyses of the published literature for survival endpoints. Statistics in Medicine 1998;17:2815-34.

\section{Posch 2015}

Posch F, Königsbrügge O, Zielinski C, Pabinger I, Ay C. Treatment of venous thromboembolism in patients with cancer: a network meta-analysis comparing efficacy and safety of anticoagulants. Thrombosis Research 2015;136(3):582-9.

\section{Review Manager 2020 [Computer program]}

The Nordic Cochrane Centre, The Cochrane Collaboration Review Manager (RevMan). Version 5.4. Copenhagen: The Nordic Cochrane Centre, The Cochrane Collaboration, 2020.

\section{Romera-Villegas 2010}

Romera-Villegas A, Cairols-Castellote MA, Vila-Coll R, MartíMestre X, Colomé E, Iguaz I. Long-term use of different doses of low-molecular-weight heparin versus vitamin $\mathrm{K}$ antagonists in the treatment of venous thromboembolism. Annals of Vascular Surgery 2010;24(5):628-39.

\section{Santesso 2020}

Santesso N, Glenton C, Dahm P, Garner P, Akl EA, Alper B, Brignardello-Petersen R, Carrasco-Labra A, De Beer H, Hultcrantz M, Kuijpers T. GRADE guidelines 26: informative statements to communicate the findings of systematic reviews of interventions. Journal of clinical epidemiology 2020 Mar 1;119:126-35.

\section{Simmonds 2017}

Simmonds ME, Salanti G, Higgins JE, McKenzie J, Elliott JE on behalf of the Living Systematic Review Network. Living Systematic Reviews: 3. Statistical methods for updating metaanalyses. Journal of Clinical Epidemiology 2017;91:38-46.

\section{Sorensen 2000}

Sorensen HT, Mellemkjaer L, Olsen JH, Baron JA. Prognosis of cancers associated with venous thromboembolism. New England Journal of Medicine 2000;343(25):1846-50.

\section{Stata 9 [Computer program]}

Stata Statistical Software: Release 9. StataCorp. College Station, TX: StataCorp LP, 2005.

\section{Synnot 2017}

Synnot A, Turner T, Elliott J, Akl E, MacLehose $\mathrm{H}$ and the Living Systematic Review Network. Cochrane Living Systematic Reviews Interim guidance for pilots (Version 0.3, 21 April 2017). community.cochrane.org/review-production/productionresources/living-systematic-reviews (accessed prior to 3 November 2017).

\section{Thomas 2017}

Thomas J, Noel-Storr A, Marshall I, Wallace B, McDonald S, Mavergames C, Glasziou P, Shemilt I, Synnot A, Turner T, Elliott J. Living systematic reviews: 2. Combining human and machine effort. Journal of clinical epidemiology 2017 Nov 1;91:31-7. 


\section{van der Heijden 2007}

van der Heijden JF, Hutten BA, Büller HR, Prins MH. Vitamin $\mathrm{K}$ antagonists or low-molecular-weight heparin for the long term treatment of symptomatic venous thromboembolism. Cochrane Database of Systematic Reviews 2007, Issue 3. Art. No: CD002001. [DOI: 10.1002/14651858.CD002001]

\section{Vedovati 2015}

Vedovati MC, Germini F, Agnelli G, Becattini C. Direct oral anticoagulants in patients with VTE and cancer: a systematic review and meta-analysis. Chest Journal 2015;147(2):475-83.

\section{Wallace 2017}

Wallace BC, Noel-Storr A, Marshall IJ, Cohen AM, Smalheiser NR, Thomas J. Identifying reports of randomized controlled trials (RCTs) via a hybrid machine learning and crowd sourcing approach. Journal of the American Medical Informatics Association 2017;0(0):1-4.

\section{References to other published versions of this review Akl 2007}

Akl EA, Barba M, Schünemann HJ, Sperati F, Terrenato I, Muti P, et al. Anticoagulation for the long term treatment of venous thromboembolism in patients with cancer. Cochrane Database of Systematic Reviews 2007, Issue 3. Art. No: CD006650. [DOI: 10.1002/14651858.CD006650]

\section{Akl 2008}

Akl EA, Barba M, Rohilla S, Terrenato I, Sperati F, Muti P, et al. Anticoagulation for the long term treatment of venous thromboembolism in patients with cancer. Cochrane Database of Systematic Reviews 2008, Issue 2. Art. No: CD006650. [DOI: 10.1002/14651858.CD006650.pub2]

\section{Akl 2011}

Akl EA, Labedi N, Barba M, Terrenato I, Sperati F, Muti P, et al. Anticoagulation for the long-term treatment of venous thromboembolism in patients with cancer. Cochrane Database of Systematic Reviews 2011, Issue 6. Art. No: CD006650. [DOI: 10.1002/14651858.CD006650.pub3]

\section{Akl 2014b}

Akl EA, Kahale LA, Barba M, Neumann I, Labedi N, Terrenato I, et al. Anticoagulation for the long-term treatment of venous thromboembolism in patients with cancer. Cochrane Database of Systematic Reviews 2014, Issue 7. Art. No: CD006650. [DOI: 10.1002/14651858.CD006650.pub4]

\section{Kahale 2018}

Kahale LA, HakoumMB, Tsolakian IG, Matar CF, Terrenato I, Sperati F, Barba M, Yosuico VED, Schünemann H, Akl EA. Anticoagulation for the long-termtreatment of venous thromboembolism in people with cancer. Cochrane Database of Systematic Reviews 2018, Issue 6. Art. No: CD006650. [DOI: 10.1002/14651858.CD006650.pub5]

* Indicates the major publication for the study

\section{CHARACTERISTICS OF STUDIES}

Characteristics of included studies [ordered by study ID]

Agnelli 2015 (AMPLIFY)

\section{Study characteristics}

\begin{tabular}{ll}
\hline Methods & Multicenter randomized double-blind trial \\
\hline Participants & $\begin{array}{l}169(3.1 \%) \text { participants with active cancer at baseline with objectively confirmed symptomatic proxi- } \\
\text { mal DVT or PE, or both from } 358 \text { centers in } 28 \text { countries }\end{array}$ \\
& $\begin{array}{l}\text { Mean age } 65.3 \text { years, } 58.5 \% \text { male, } 1 / 3 \text { had metastatic disease. Most common cancer sites were prostate, } \\
\text { breast, colon, bladder and lung }\end{array}$
\end{tabular}

Interventions Intervention: apixaban (10 mg twice daily for 7 days followed by $5 \mathrm{mg}$ twice daily) for a total of 6 months

Control: enoxaparin ( $1 \mathrm{mg} / \mathrm{kg}$ twice daily for at least 5 days) and warfarin (target INR 2-3) starting day 2 of enoxaparin for a total of 6 months

Discontinued treatment: not reported for subgroup of participants with active cancer

Duration of follow-up for the following outcomes: 6 months
- All-cause mortality
- Recurrent VTE
- Major bleeding


Agnelli 2015 (AMPLIFY) (Continued)

- Clinically relevant non-major bleeding

Screening test for DVT/PE: not reported

Diagnostic test for DVT/PE: echo-doppler for DVT and spiral CT scan for PE

- Study details obtained from original AMPLIFY report published in New England Journal of Medicine
August 2013.
- Participants with cancer history at baseline and without active cancer at baseline and participants
with no cancer history and no active cancer at baseline were excluded from this meta-analysis.
- Funded by Bristol-Myers Squibb and Pfizer Inc.
- Ethical approval: not reported
- Conflict of interest: more than one author reported financial COI
- ITT: "All efficacy analyses included data for patients in the intention-to-treat population for whom the
outcome status at 6 months was documented."

\section{Risk of bias}

\begin{tabular}{|c|c|c|}
\hline Bias & Authors' judgement & Support for judgement \\
\hline $\begin{array}{l}\text { Random sequence genera- } \\
\text { tion (selection bias) }\end{array}$ & Low risk & $\begin{array}{l}\text { Quote: "Randomization was performed with the use of an interactive voice-re- } \\
\text { sponse system" }\end{array}$ \\
\hline $\begin{array}{l}\text { Allocation concealment } \\
\text { (selection bias) }\end{array}$ & Low risk & $\begin{array}{l}\text { Quote: "interactive voice-response system" } \\
\text { Comment: Definitely concealed }\end{array}$ \\
\hline $\begin{array}{l}\text { Blinding of participants } \\
\text { and personnel (perfor- } \\
\text { mance bias) } \\
\text { All outcomes }\end{array}$ & Low risk & $\begin{array}{l}\text { Quote: "AMPLIFY was a randomised, double-blind trial." } \\
\text { Comment: definitely blinded }\end{array}$ \\
\hline $\begin{array}{l}\text { Blinding of outcome as- } \\
\text { sessment (detection bias) } \\
\text { All outcomes }\end{array}$ & Low risk & $\begin{array}{l}\text { Quote: "All efficacy and safety outcomes were adjudicated by an independent } \\
\text { committee blinded to treatment assignment." } \\
\text { Comment: definitely blinded }\end{array}$ \\
\hline $\begin{array}{l}\text { Incomplete outcome data } \\
\text { (attrition bias) } \\
\text { All outcomes }\end{array}$ & Low risk & $\begin{array}{l}\text { No information about follow-up in the cancer subgroup reported } \\
\text { comment: probably complete follow-up }\end{array}$ \\
\hline $\begin{array}{l}\text { Free of selective report- } \\
\text { ing? }\end{array}$ & Low risk & $\begin{array}{l}\text { Study not registered. All outcomes listed in the protocol and methods section } \\
\text { of this study were reported on in the results section. }\end{array}$ \\
\hline Free of other bias? & Low risk & Study not reported as stopped early for benefit \\
\hline
\end{tabular}

Agnelli 2020 (Caravaggio)

\section{Study characteristics}

\begin{tabular}{ll}
\hline Methods & Multinational, randomized, investigator-initiated, open-label, non-inferiority trial. \\
\hline Participants & 1155 patients with active cancer from 119 centers in 9 European countries, Israel and the United States. \\
& Mean age 67.2 years, $49.2 \%$ male, $68 \%$ had metastatic disease, $9.2 \%$ had previous VTE.
\end{tabular}


Agnelli 2020 (Caravaggio) (Continued)

Both solid and hematological malignancy. The most frequent solid tumours were: colorectal (about $20 \%$ ), lung (about 17\%) and breast (about 13\%).

Interventions

Intervention: Apixaban (10 mg twice daily for 7 days and $5 \mathrm{mg}$ twice daily) for a total of 6 months

Control: Dalteparin (200IU per kilogram of body weight once daily for the fist months, after which the dose was reduced to 150 IU per kilogram daily).

Discontinued treatment: $38 / 576(6.6 \%)$ in the intervention arm and 48/579 (8.3\%)

Duration of follow-up for the following outcomes: 6 months
- All-cause mortality (at 7 months)
- Recurrent VTE, DVT, PE
- Major bleeding (gastointestinal and nongastrointestinal)
- Clinically relevant non-major bleeding
Screening test for DVT/PE: CT pulmonary angiography.
Diagnostic test for DVT/PE: For DVT ultrasonography, venography, CT venography, or MR venography.
For PE CT pulmonary angiography/angiogram or VQ scan.
- Funded by Bristol-Myers Squibb and Pfizer Inc.The Bristol-Myers Squibb-Pfizer Alliance played no role
in the design or conduct of the trial, the collection or analysis of the data, or the review or editing of
the manuscript.
- Ethical approval: The protocol and its amendments were approved by the ethics committee at each
trial center.
- Conflict of interest: More than one author reported reported financial conflict of interest
ITT: No formal interim analysis was planned. The primary efficacy data set (modified intention-to-treat
population) and safety data set consisted of all the patients who had undergone. randomization and
received at least one dose of a trial drug. The secondary efficacy data set consisted of all the patients
who had undergone randomization (intention-to-treat population) alongwith the per-protocol popu-
lation; the latter consisted of all the patients in the intention-to-treat population who completed the
trial in full compliance with the protocol and without any major deviation.

\section{Risk of bias}

\begin{tabular}{lll}
\hline Bias & Authors' judgement & Support for judgement \\
\hline $\begin{array}{l}\text { Random sequence genera- } \\
\text { tion (selection bias) }\end{array}$ & Low risk & $\begin{array}{l}\text { Quote:"Randomization was centrally performed through an interactive online } \\
\text { system and stratified according to..." }\end{array}$ \\
\hline $\begin{array}{l}\text { Allocation concealment } \\
\text { (selection bias) }\end{array}$ & Low risk & $\begin{array}{l}\text { Quote:"Randomization was centrally performed through an interactive online } \\
\text { system and stratified according to..." }\end{array}$ \\
\hline $\begin{array}{l}\text { Blinding of participants } \\
\text { and personnel (perfor- } \\
\text { mance bias) }\end{array}$ & High risk & $\begin{array}{l}\text { Quote: "This trial was a multinational, randomized, controlled, investiga- } \\
\text { tor-initiated, open-label, noninferiority trial with blinded adjudication of the } \\
\text { outcomes" }\end{array}$ \\
& $\begin{array}{l}\text { Comment: probably not blinded; knowledge of the assigned intervention may } \\
\text { have led to differential behaviors across intervention groups (e.g. differential } \\
\text { dropout, differential cross-over to an alternative intervention or differential } \\
\text { administration of cointerventions) }\end{array}$
\end{tabular}

\begin{tabular}{|c|c|c|}
\hline $\begin{array}{l}\text { Blinding of outcome as- } \\
\text { sessment (detection bias) } \\
\text { All outcomes }\end{array}$ & Low risk & $\begin{array}{l}\text { Quote: "This trial was a multinational, randomized, controlled, investiga- } \\
\text { tor-initiated, open-label, noninferiority trial with blinded adjudication of the } \\
\text { outcomes }\end{array}$ \\
\hline
\end{tabular}

Comment: judgment based on comparison in the intervention arm between

Incomplete outcome data Low risk rate of participants with missing data $(24 / 585(4.1 \%))$ and event rate $(135 / 585$

(attrition bias)

Anticoagulation for the long-term treatment of venous thromboembolism in people with cancer (Review) 
Agnelli 2020 (Caravaggio) (Continued)

All outcomes

Free of selective report- Low risk
ing?
ing?
(24\%)) for the main outcome-mortality. Similary for the control arm: rate of participants with missing data $(17 / 585(3 \%))$ and event rate $(153 / 568(27 \%))$.

Free of other bias? Low risk Study not reported as stopped early for benefit.

\section{Cesarone 2003}

\section{Study characteristics}

\begin{tabular}{ll}
\hline Methods & Randomized trial (abstract) \\
\hline Participants & 199 participants with cancer with DVT \\
& 17 dropouts, 182 participants completed study \\
\hline
\end{tabular}

Interventions

Intervention: enoxaparin $100 \mathrm{lU} / \mathrm{kg}$ twice daily $\times 3$ months

Control: coumadin (target INR 3 ) $\times 3$ months

Discontinued treatment: $17 / 199(8.5 \%)$ in both arms

\begin{tabular}{ll}
\hline Outcomes & Duration of follow-up for the following outcomes: 3 months \\
- Mortality \\
- Major bleeding \\
- Recurrent DVT or PE but no data available \\
Screening test for DVT/PE: not reported \\
Diagnostic test for DVT/PE: ultrasound \\
\hline Notes \\
- Funding: not reported \\
- Conflict of interest: not reported \\
- ITT: not reported
\end{tabular}

\section{Risk of bias}

\begin{tabular}{lll}
\hline Bias & Authors' judgement & Support for judgement \\
\hline $\begin{array}{l}\text { Random sequence genera- } \\
\text { tion (selection bias) }\end{array}$ & Low risk & $\begin{array}{l}\text { Quote: "randomised outpatient trial" } \\
\text { Comment: probably generated sequence randomly }\end{array}$ \\
\hline $\begin{array}{l}\text { Allocation concealment } \\
\text { (selection bias) }\end{array}$ & High risk & Not reported \\
\hline $\begin{array}{l}\text { Blinding of participants } \\
\text { and personnel (perfor- } \\
\text { mance bias) } \\
\text { All outcomes }\end{array}$ & High risk & Comment: probably not done \\
\hline
\end{tabular}


dropout, differential cross-over to an alternative intervention or differential administration of cointerventions).

\begin{tabular}{|c|c|c|}
\hline Blinding of outcome as- & Low risk & Not reported \\
\hline $\begin{array}{l}\text { Sessment (detection bias } \\
\text { All outcomes }\end{array}$ & & $\begin{array}{l}\text { Comment: probably not blinded; knowledge of the assigned intervention may } \\
\text { not have impacted the assessment of the physiologic outcomes (mortality, } \\
\text { DVT, PE, bleeding, etc.). }\end{array}$ \\
\hline
\end{tabular}

\begin{tabular}{lll}
\hline Incomplete outcome data & High risk & Comment: judgment based on comparison between rate of participants with \\
(attrition bias) & missing data $(17 / 199(8.5 \%))$ and event rate for mortality $1 / 185(0.5 \%)$ and for \\
All outcomes & major outcome $19 / 185(10 \%)$ across both arms
\end{tabular}

\begin{tabular}{|c|c|c|}
\hline $\begin{array}{l}\text { Free of selective report- } \\
\text { ing? }\end{array}$ & High risk & $\begin{array}{l}\text { Quote: "in the OC [oral coumadin] group } 14 \text { subjects (16.3\%) experienced one } \\
\text { major outcome event compared with } 5 \text { patients ( } 5.2 \%) \text { out of } 96 \text { in the LMWH" } \\
\text { Outcomes mentioned in the methods section (DVT, PE, major bleeding) not re- } \\
\text { ported in the results section }\end{array}$ \\
\hline Free of other bias? & High risk & $\begin{array}{l}\text { Study not reported as stopped early for benefit. The full-text of the study was } \\
\text { never published. }\end{array}$ \\
\hline
\end{tabular}

Deitcher 2006 (ONCENOX)

\section{Study characteristics}

\begin{tabular}{|c|c|}
\hline Methods & Randomized clinical trial \\
\hline \multirow[t]{2}{*}{ Participants } & 102 participants with active cancer with DVT, PE, or both \\
\hline & $85 \%$ Caucasian, mean age 64 years, $46 \%$ male, $8.7 \%$ had previous VTE \\
\hline \multirow[t]{4}{*}{ Interventions } & $\begin{array}{l}\text { Intervention: enoxaparin } 1 \mathrm{mg} / \mathrm{kg} \text { twice daily } \times 5 \text { days followed by } 1.0-1.5 \mathrm{mg} / \mathrm{kg} \text { daily } \times 175 \text { days } \\
\text { (group 1a); enoxaparin } 1.5 \mathrm{mg} / \mathrm{kg} \text { daily } \times 175 \text { days (group } 1 \mathrm{~b} \text { ) }\end{array}$ \\
\hline & $\begin{array}{l}\text { Control: enoxaparin for a minimum of } 5 \text { days and until achievement of a stable INR 2-3 on oral warfarin } \\
\text { begun on day } 2 \text { of enoxaparin and continued for a total of } 180 \text { days of anticoagulation }\end{array}$ \\
\hline & Cointervention: chemotherapy, radiation therapy, or both (not better specified) \\
\hline & Discontinued treatment: $43 / 98(63 \%)$ in the intervention arm and $21 / 34(61 \%)$ in the control arm \\
\hline \multirow[t]{6}{*}{ Outcomes } & Duration of follow-up for the following outcomes: 1 year \\
\hline & - Mortality \\
\hline & - Symptomatic recurrent VTE \\
\hline & - Major bleeding \\
\hline & - Minor bleeding \\
\hline & Diagnostic test for DVT/PE: not reported \\
\hline \multirow[t]{4}{*}{ Notes } & - Funding: Aventis Pharmaceutical \\
\hline & $\begin{array}{l}\text { - Ethical approval: "The appropriate institutional review board at each investigative site approved this } \\
\text { study." }\end{array}$ \\
\hline & - Conflict of interest: not reported \\
\hline & - ITT: "patients in the intent-to-treat population" \\
\hline
\end{tabular}


Deitcher 2006 (ONCENOX) (Continued)

Risk of bias

\begin{tabular}{|c|c|c|}
\hline Bias & Authors' judgement & Support for judgement \\
\hline $\begin{array}{l}\text { Random sequence genera- } \\
\text { tion (selection bias) }\end{array}$ & Low risk & $\begin{array}{l}\text { Quote: "patients were randomly allocated" } \\
\text { Comment: probably generated sequence randomly }\end{array}$ \\
\hline $\begin{array}{l}\text { Allocation concealment } \\
\text { (selection bias) }\end{array}$ & High risk & $\begin{array}{l}\text { Not reported } \\
\text { Comment: probably not done }\end{array}$ \\
\hline $\begin{array}{l}\text { Blinding of participants } \\
\text { and personnel (perfor- } \\
\text { mance bias) } \\
\text { All outcomes }\end{array}$ & High risk & $\begin{array}{l}\text { Open-label trial } \\
\text { Comment: definitely not blinded; knowledge of the assigned intervention may } \\
\text { have led to differential behaviors across intervention groups (e.g. differential } \\
\text { dropout, differential cross-over to an alternative intervention or differential } \\
\text { administration of cointerventions). }\end{array}$ \\
\hline $\begin{array}{l}\text { Blinding of outcome as- } \\
\text { sessment (detection bias) } \\
\text { All outcomes }\end{array}$ & Low risk & $\begin{array}{l}\text { Open-label trial } \\
\text { Comment: probably not blinded; knowledge of the assigned intervention may } \\
\text { not have impacted the assessment of the physiological outcomes (mortality, } \\
\text { DVT, PE, bleeding, etc.). }\end{array}$ \\
\hline $\begin{array}{l}\text { Incomplete outcome data } \\
\text { (attrition bias) } \\
\text { All outcomes }\end{array}$ & Low risk & $\begin{array}{l}\text { Comment: judgment based on comparison in the intervention arm between } \\
\text { rate of participants with missing data }(15 / 68(22 \%)) \text { and event rate }(22 / 53 \\
(41 \%)) \text { for the main outcome-mortality. Similary for the control arm: rate of } \\
\text { participants with missing data }(2 / 34(6 \%)) \text { and event rate }(11 / 32(34 \%)) \text {. }\end{array}$ \\
\hline $\begin{array}{l}\text { Free of selective report- } \\
\text { ing? }\end{array}$ & Low risk & $\begin{array}{l}\text { Study not registered and no published protocol identified. All relevant out- } \\
\text { comes listed in the methods section were reported on in the results section. }\end{array}$ \\
\hline Free of other bias? & Low risk & Study not reported as stopped early for benefit \\
\hline
\end{tabular}

\section{El Mokadem 2020}

\section{Study characteristics}

\begin{tabular}{ll}
\hline Methods & Randomized clinical study. \\
\hline Participants & 138 particpants with active cancer and acute VTE from the oncology outpatient clinic of Beni-Suef Uni- \\
versity hospital Egypt. \\
Mean age 60 years; $42 \%$ men, $84 \%$ had metastatic disease, $100 \%$ solid malignancy, $42 \%$ had colon can- \\
cer
\end{tabular}

Interventions Intervention: Apixaban $10 \mathrm{mg}$ twice daily dose for seven days followed by apixaban $5 \mathrm{mg}$ twice daily. Apixaban dose was adjusted to be $2.5 \mathrm{mg}$ twice daily in patients with serum creatinine $1.5 \mathrm{mg} / \mathrm{dL}$, elderly patients 80 years or those with body weight $60 \mathrm{~kg}$. Apixaban $10 \mathrm{mg}$ twice daily for seven days followed by $5 \mathrm{mg}$ twice daily for six months.

Control: Enoxaparin (1 mg/kg/SC every $12 \mathrm{~h})$.

Discontinued treatment: $4 / 69(5.8 \%)$ in the intervention arm and 8/69 (11.6\%) in the control arm

Outcomes Duration of follow-up for the following outcomes: 6 months

- Mortality 
El Mokadem 2020 (Continued)

- Recurrence DVT

- Recurrence PE

- Major bleeding

- Minor bleeding

Screening test for DVT/PE: venous doppler ultrasound.

Diagnostic test for DVT/PE: venous doppler ultrasound.

- Notes
- Ethical approval: The protocol was approved by the ethics committee.
- Conflict of interest: The authors declare no competing financial interest.
- ITT: The intention-to-treat analysis defined included all the patients who had undergone randomiza-
tion.

\section{Risk of bias}

\begin{tabular}{lll}
\hline Bias & Authors' judgement & Support for judgement \\
\hline $\begin{array}{l}\text { Random sequence genera- } \\
\text { tion (selection bias) }\end{array}$ & Low risk & $\begin{array}{l}\text { Quote:" Computer based program (Random number generators) using } \\
\text { Math.random". }\end{array}$ \\
\hline $\begin{array}{ll}\text { Allocation concealment } \\
\text { (selection bias) }\end{array}$ & High risk & Not reported. \\
& & Comment: probably not concealed \\
\hline
\end{tabular}

\section{Blinding of participants High risk} and personnel (perfor-

mance bias)

All outcomes

Comment: probably not blinded; knowledge of the assigned intervention may have led to differential behaviors across intervention groups (e.g. differential dropout, differential cross-over to an alternative intervention or differential administration of cointerventions)

\begin{tabular}{|c|c|c|}
\hline $\begin{array}{l}\text { Blinding of outcome as- } \\
\text { sessment (detection bias) }\end{array}$ & Low risk & Not reported. \\
\hline All outcomes & & $\begin{array}{l}\text { Comment: probably not blinded; knowledge of the assigned intervention may } \\
\text { not have impacted the assessment of the physiological outcomes (mortality, } \\
\text { DVT, PE, bleeding, etc.). }\end{array}$ \\
\hline
\end{tabular}

$\begin{array}{lll}\begin{array}{l}\text { Incomplete outcome data } \\ \text { (attrition bias) }\end{array} & \text { High risk } & \begin{array}{l}\text { Comment: judgment based on comparison in the intervention arm between } \\ \text { rate of participants with missing data }(4 / 69(5.7 \%)) \text { and event rate }(3 / 50(6 \%)) \\ \text { fll outcomes }\end{array} \\ & \begin{array}{l}\text { for the outcome recurrent VTE. Similary for the control arm: rate of partici- } \\ \text { pants with missing data }(8 / 68(11.6 \%)) \text { and event rate }(5 / 50(10 \%)) .\end{array}\end{array}$

$\begin{array}{ll}\begin{array}{l}\text { Free of selective report- } \\ \text { ing? }\end{array} & \text { Low risk } \\ \end{array}$

Free of other bias? Low risk Study not reported as stopped early for benefit. Monocentric study.

\section{Hull 2006 (LITE)}

\section{Study characteristics}

\begin{tabular}{ll}
\hline Methods & Randomized clinical trial \\
\hline Participants & 200 participants with cancer (solid or hematologic) with proximal DVT with or without PE \\
\hline
\end{tabular}


Hull 2006 (LITE) (Continued)

Minimum age 18 years, minimum life expectancy 3 months, 50\% men, 19\% had previous VTE

Interventions Interventions: tinzaparin 175 anti-Xa/kg SC daily for 12 weeks

Control: UFH either $5000 \mathrm{U}$ or $80 \mathrm{U} / \mathrm{kg}$ for 5 days followed by VKAs (target INR 2-3) for 12 weeks

Discontinued treatment: $1 / 100(1 \%)$ in the intervention arm and $1 / 100(1 \%)$ in the control arm

Outcomes
- Moration of follow-up for the following outcomes: 12 months
- Recurrent VTE evaluated at 3 and 12 months
- Bleeding (major and minor) evaluated at 3 months
- Thtombocytopenia evaluated at 3 months
Screening test for DVT/PE: not reported
Diagnostic test for recurrent VTE: venography or compression ultrasonography
- Funding: Canadian Institute for Health Research, industry grant, Leo Pharmaceutical, Pharmion Phar-
- maceutical and DuPont Pharmaceutical
- Ethical approval: "The protocol was approved by the institutional review board at each center."
- ITT: not reported

\section{Risk of bias}

\begin{tabular}{lll}
\hline Bias & Authors' judgement & Support for judgement \\
\hline $\begin{array}{l}\text { Random sequence genera- } \\
\text { tion (selection bias) }\end{array}$ & Low risk & $\begin{array}{l}\text { Quote: "a computer-derived randomised treatment schedule was used; within } \\
\text { the each stratum, the randomised schedule was balanced in blocks of } 2 \text { and 4." }\end{array}$ \\
\hline $\begin{array}{l}\text { Allocation concealment } \\
\text { (selection bias) }\end{array}$ & High risk & Not reported \\
& & Comment: probably not concealed \\
\hline
\end{tabular}

\begin{tabular}{|c|c|c|}
\hline $\begin{array}{l}\text { Blinding of participants } \\
\text { and personnel (perfor- } \\
\text { mance bias) } \\
\text { All outcomes }\end{array}$ & High risk & $\begin{array}{l}\text { Open-label trial } \\
\text { Comment: definitely not blinded; knowledge of the assigned intervention may } \\
\text { have led to differential behaviors across intervention groups (e.g. differential } \\
\text { dropout, differential cross-over to an alternative intervention or differential } \\
\text { administration of cointerventions). }\end{array}$ \\
\hline
\end{tabular}

\begin{tabular}{|c|c|c|}
\hline $\begin{array}{l}\text { Blinding of outcome as- } \\
\text { sessment (detection bias) } \\
\text { All outcomes }\end{array}$ & Low risk & $\begin{array}{l}\text { Quote: "Adjudication was made by } 2 \text { committee members not involved in the } \\
\text { patient's care, and disputes were resolved independently by a third. Members } \\
\text { of the committee were unaware of the patients' treatment assignments." }\end{array}$ \\
\hline & & Comment: probably yes \\
\hline
\end{tabular}

\begin{tabular}{ll}
\hline $\begin{array}{l}\text { Incomplete outcome data } \\
\text { (attrition bias) }\end{array}$ & Low risk \\
All outcomes & $\begin{array}{l}\text { Comment: judgment based on comparison in the intervention arm between } \\
\text { rate of participants with missing data }(1 / 100(1 \%)) \text { and event rate }(20 / 99(20 \%)) \\
\text { for the outcome mortality. Similary for the control arm: rate of participants } \\
\text { with missing data }(1 / 100(1 \%)) \text { and event rate } 19 / 99(19 \%)) .\end{array}$ \\
\hline
\end{tabular}

\begin{tabular}{|c|c|c|}
\hline $\begin{array}{l}\text { Free of selective report- } \\
\text { ing? }\end{array}$ & Low risk & $\begin{array}{l}\text { Study not registered. No published protocol identified but a protocol was } \\
\text { clearly mentioned in the discussion. All relevant outcomes listed in the meth- } \\
\text { ods section were reported on in the results section. }\end{array}$ \\
\hline
\end{tabular}

Free of other bias? Low risk Study not reported as stopped early for benefit


Lee 2003 (CLOT)

\section{Study characteristics}

Methods Randomized clinical trial

Participants 676 participants with active cancer and with DVT, PE, or both; ECOG 1 or 2
Mean age 63 years, $49 \%$ male, $11 \%$ had history of DVT/PE

Interventions Intervention: dalteparin $200 \mathrm{IU} / \mathrm{kg}$ daily $\times 1$ month followed by $150 \mathrm{IU} / \mathrm{kg}$ daily $\times 5$ months

Control: dalteparin $200 \mathrm{lU} / \mathrm{kg}$ daily $\times$ 5-7 days followed by warfarin or acenocoumarol (target INR 2-3) $\times$ 6 months; $46 \%$ of time on target

Discontinued treatment: $2 / 338(0.5 \%)$ in the intervention arm and $2 / 338(0.5 \%)$ in the control arm

Duration of follow-up for the following outcomes: 6 months
- Mortality
- Symptomatic recurrent DVT and PE
- Clinically overt bleeding (both major bleeding and any bleeding)

Screening test for DVT/PE: not reported

Diagnostic test for DVT: ultrasonography, venography

Diagnostic test for PE: lung scan, angiography, autopsy

Notes

- Funding: Pharmacia

- Ethical approval: the study protocol was reviewed and approved by the institutional review boards of each participating center

- Conflict of interest: More than one author reported financial conflict of interest.

- ITT: "analysis was performed according to intention to treat principle."

\section{Risk of bias}

\begin{tabular}{|c|c|c|}
\hline Bias & Authors' judgement & Support for judgement \\
\hline $\begin{array}{l}\text { Random sequence genera- } \\
\text { tion (selection bias) }\end{array}$ & Low risk & $\begin{array}{l}\text { Quote: "randomizations was stratified according to the clinical center and cen- } \\
\text { tralized at the coordinating and methods center." }\end{array}$ \\
\hline $\begin{array}{l}\text { Allocation concealment } \\
\text { (selection bias) }\end{array}$ & Low risk & $\begin{array}{l}\text { Quote: "randomizations was stratified according to the clinical center and cen- } \\
\text { tralized at the coordinating and methods center." }\end{array}$ \\
\hline $\begin{array}{l}\text { Blinding of participants } \\
\text { and personnel (perfor- } \\
\text { mance bias) } \\
\text { All outcomes }\end{array}$ & High risk & $\begin{array}{l}\text { Open-label trial } \\
\text { Comment: definitely not blinded; knowledge of the assigned intervention may } \\
\text { have led to differential behaviors across intervention groups (e.g. differential }\end{array}$ \\
\hline
\end{tabular}


Lee 2003 (CLOT) (Continued)

dropout, differential cross-over to an alternative intervention or differential administration of cointerventions).

\begin{tabular}{|c|c|c|}
\hline $\begin{array}{l}\text { Blinding of outcome as- } \\
\text { sessment (detection bias) } \\
\text { All outcomes }\end{array}$ & Low risk & $\begin{array}{l}\text { Quote: "all suspected events were reviewed by a central adjudication commit- } \\
\text { tee whose members were unaware of the patient's treatment assignments." } \\
\text { Comment: definitely blinded }\end{array}$ \\
\hline $\begin{array}{l}\text { Incomplete outcome data } \\
\text { (attrition bias) } \\
\text { All outcomes }\end{array}$ & Low risk & $\begin{array}{l}\text { Comment: judgment based on comparison in the intervention arm between } \\
\text { rate of participants with missing data }(2 / 338(0.5 \%)) \text { and event rate }(120 / 336 \\
(38.7 \%)) \text { for the outcome mortality. Similary for the control arm: rate of partici- } \\
\text { pants with missing data } 2 / 338(0.5 \%)) \text { and event rate } 136 / 336(40.4 \%)) \text {. }\end{array}$ \\
\hline $\begin{array}{l}\text { Free of selective report- } \\
\text { ing? }\end{array}$ & Low risk & $\begin{array}{l}\text { Study not registered and no published protocol identified. All relevant out- } \\
\text { comes listed in the methods section were reported on in the results section. }\end{array}$ \\
\hline Free of other bias? & Low risk & $\begin{array}{l}\text { Study not reported as stopped early for benefit } \\
\text { No other bias suspected }\end{array}$ \\
\hline
\end{tabular}

Lee 2015 (CATCH)

\section{Study characteristics}

$\begin{array}{ll}\text { Methods } & \begin{array}{l}\text { Phase III, multinational, concealed, randomized, active-controlled, open-label trial with blinded adju- } \\ \text { dication }\end{array}\end{array}$
dication

Participants 900 randomized participants (adults with active cancer and acute proximal DVT, PE, or both)
Mean age $59 \%, 40 \%$ men, 22\% gynecologic cancer

Interventions Intervention: LMWH (tinzaparin) $175 \mathrm{IU} / \mathrm{kg}$ SC once daily for 180 days (almost 6 months)

Control: VKA (warfarin) for 6 months, overlapping with tinzaparin $175 \mathrm{IU} / \mathrm{kg}$ once daily (first 5-10 days and until INR $>2$ for 2 consecutive days)

Discontinued treatment: $140 / 449(\%)$ in the intervention arm and 172/451 (\%) in the control arm

Outcomes Duration of follow-up for the following outcomes: every 30 days until day 180

- Symptomatic DVT

- Symptomatic non-fatal PE

- Fatal PE

- Incidental proximal DVT (popliteal vein or higher)

- Incidental proximal PE (segmental arteries or larger)

Duration of follow-up for the following outcomes: until 1 month following last dose of study treatment

- All-cause mortality

- Major bleeding

- Clinically relevant non-major bleeding

- Heparin-induced thrombocytopenia

Screening test for DVT/PE: not reported

Diagnostic test for DVT: ultrasonography, venography, CT venography or magnetic resonance venography 
Diagnostic test for PE: ventilation/perfusion scintigraphy, standard pulmonary angiography or CT

Notes

- NCT01130025

- Funding: LEO Pharma

- Ethical approval: "Institutional ethics approval was obtained at each participating center."

- Conflict of interest: More than one author reported financial COI

- ITT: "patients were randomised and included in intention-to-treat efficacy and safety analysis."

\section{Risk of bias}

\begin{tabular}{|c|c|c|}
\hline Bias & Authors' judgement & Support for judgement \\
\hline $\begin{array}{l}\text { Random sequence genera- } \\
\text { tion (selection bias) }\end{array}$ & Low risk & $\begin{array}{l}\text { Quote: "treatment assignment was planned according to a computer-generat- } \\
\text { ed randomisation schedule 1:1 in a ratio." }\end{array}$ \\
\hline $\begin{array}{l}\text { Allocation concealment } \\
\text { (selection bias) }\end{array}$ & Low risk & $\begin{array}{l}\text { Quote: "concealed until individual randomisation using an interactive voice- } \\
\text { response system" }\end{array}$ \\
\hline $\begin{array}{l}\text { Blinding of participants } \\
\text { and personnel (perfor- } \\
\text { mance bias) } \\
\text { All outcomes }\end{array}$ & High risk & $\begin{array}{l}\text { Open-label study } \\
\text { Comment: definitely not blinded; knowledge of the assigned intervention may } \\
\text { have led to differential behaviors across intervention groups (e.g. differential } \\
\text { dropout, differential cross-over to an alternative intervention or differential } \\
\text { administration of cointerventions). }\end{array}$ \\
\hline $\begin{array}{l}\text { Blinding of outcome as- } \\
\text { sessment (detection bias) } \\
\text { All outcomes }\end{array}$ & Low risk & $\begin{array}{l}\text { Quote: "Members of a central, independent adjudication committee, who were } \\
\text { unaware of the study treatment assignments, reviewed and adjudicated all } \\
\text { suspected cases of recurrent VTE, heparin-induced thrombocytopenia (HIT), } \\
\text { bleeding events, and causes of death." }\end{array}$ \\
\hline
\end{tabular}

Incomplete outcome data High risk

(attrition bias)

All outcomes

Comment: judgment based on comparison in the intervention arm between rate of participants with missing data $(33 / 449(7.3 \%))$ and event rate $(6.9 \%))$ for the outcome recurrent VTE. Similary for the control arm: rate of participants with missing data (50/451 (11\%)) and event rate 136/336 (10\%))

$\begin{array}{ll}\begin{array}{ll}\text { Free of selective report- } & \text { High risk }\end{array} & \begin{array}{l}\text { Protocol available. Not all outcomes listed in the protocol were reported on } \\ \text { ing? }\end{array} \\ & \begin{array}{l}\text { (such as other assessments: post-thrombotic syndrome, HRQoL, VTE risk fac- } \\ \text { tors, healthcare resource utilization). }\end{array}\end{array}$

Free of other bias? Low risk Study not reported as stopped early for benefit

No other bias suspected

Lopez-Beret 2001

\section{Study characteristics}

\begin{tabular}{ll}
\hline Methods & Randomized clinical trial \\
\hline Participants & 35 participants with known malignancy; treated for symptomatic DVT of the lower limbs
\end{tabular}


Lopez-Beret 2001 (Continued)

Minimum age 18 years, mean age 65.7 years

Interventions Intervention: nadroparin $1.025 \mathrm{AXa}$ IU/10 kg twice daily for 3 days then randomised to nadroparin 1.025 antiXa IU/10 kg twice daily After the 3rd month, nadroparin was switched to once daily

Control: nadroparin 1.025 AXa IU/10 kg twice daily for 3 days then randomised to acenocoumarol (target INR 2-3) for 3-6 months. . 68\% of INR values were on target

Discontinued treatment: not reported

Duration of follow-up for the following outcomes: 12 months
- Mortality
- Symptomatic recurrence or progression of VTE
- Bleeding
Screening test for DVT/PE: not reported
Diagnostic test for DVT: duplex scan examination

Notes

- Funding: not reported

- Ethical approval: "The study protocol was approved by the Hospital Ethics Committee"

- Conflict of interest: "Competition of interest: nil."

- ITT: not reported

\section{Risk of bias}

\begin{tabular}{lll}
\hline Bias & Authors' judgement & Support for judgement \\
\hline $\begin{array}{l}\text { Random sequence genera- } \\
\text { tion (selection bias) }\end{array}$ & Low risk & $\begin{array}{l}\text { Quote: "patients were allocated at random on third day to receive a LMWH or } \\
\text { an OA [oral anticoagulant]" } \\
\text { Comment: Probably generated sequence randomly }\end{array}$ \\
\hline $\begin{array}{l}\text { Allocation concealment } \\
\text { (selection bias) }\end{array}$ & High risk & Not reported \\
\hline $\begin{array}{l}\text { Blinding of participants } \\
\text { and personnel (perfor- } \\
\text { mance bias) } \\
\begin{array}{l}\text { All outcomes } \\
\text { Comment: probably not concealed }\end{array}\end{array}$ & High risk & $\begin{array}{l}\text { Quote: "lt was not possible to use a double design for the study" } \\
\text { Comment: definitely not blinded; knowledge of the assigned intervention may } \\
\text { lead to differential behaviours across intervention groups (for example, differ- } \\
\text { ential drop-out, differential cross-over to an alternative intervention, or differ- } \\
\text { ential administration of co-interventions) }\end{array}$
\end{tabular}

\begin{tabular}{|c|c|c|}
\hline \multirow{2}{*}{$\begin{array}{l}\text { Blinding of outcome as- } \\
\text { sessment (detection bias) } \\
\text { All outcomes }\end{array}$} & Low risk & $\begin{array}{l}\text { Quote: "the final allocation of all potential outcome events, including deaths, } \\
\text { was made by an independent panel of physicians" }\end{array}$ \\
\hline & & Comment: probably blinded \\
\hline
\end{tabular}

\begin{tabular}{|c|c|c|}
\hline $\begin{array}{l}\text { Incomplete outcome data } \\
\text { (attrition bias) }\end{array}$ & Low risk & $\begin{array}{l}\text { No information about follow-up in the cancer subgroup was reported } \\
\text { Comment: assumed complete follow-up }\end{array}$ \\
\hline
\end{tabular}

\begin{tabular}{ll}
$\begin{array}{l}\text { Free of selective report- } \\
\text { ing? }\end{array}$ & Low risk \\
\hline
\end{tabular}


Lopez-Beret 2001 (Continued)
Free of other bias?
Low risk
Study not reported as stopped early for benefit

No other bias suspected

Mazilu 2014 (OVIDIUS)

\section{Study characteristics}

\begin{tabular}{ll}
\hline Methods & Randomized controlled trial (abstract) \\
\hline Participants & 46 participants with paraneoplastic DVT \\
\hline
\end{tabular}

Interventions

Intervention: Fixed-dose dabigatran (according to individual creatinine clearance)

Control: Adjusted-dose acenocoumarol (according to individual INR determined monthly)

Discontinued treatment: not reported

Outcomes Duration of follow-up for the following outcomes: 6 months

- Mortality

- Combined outcome major bleeding or recurrent thrombosis

Screening test for DVT/PE: not reported

Diagnostic test for DVT/PE: not reported

Notes

- Funding: not reported

- Ethical approval: not reported

- Conflict of interest: not reported

- ITT: not reported

\section{Risk of bias}

\begin{tabular}{|c|c|c|}
\hline Bias & Authors' judgement & Support for judgement \\
\hline \multirow{2}{*}{$\begin{array}{l}\text { Random sequence genera- } \\
\text { tion (selection bias) }\end{array}$} & Low risk & Quote: "we randomised" \\
\hline & & Comment: probably generated sequence randomly \\
\hline $\begin{array}{l}\text { Allocation concealment } \\
\text { (selection bias) }\end{array}$ & Unclear risk & Not reported \\
\hline $\begin{array}{l}\text { Blinding of participants } \\
\text { and personnel (perfor- } \\
\text { mance bias) } \\
\text { All outcomes }\end{array}$ & Unclear risk & Not reported \\
\hline $\begin{array}{l}\text { Blinding of outcome as- } \\
\text { sessment (detection bias) } \\
\text { All outcomes }\end{array}$ & Unclear risk & Not reported \\
\hline
\end{tabular}


Mazilu 2014 (OVIDIUS) (Continued)

Incomplete outcome data Unclear risk Not reported
(attrition bias)

All outcomes

Free of selective report- Unclear risk Study not registered and no published protocol identified
ing?

\begin{tabular}{ll}
\hline Free of other bias? & Unclear risk \\
& No other bias suspected
\end{tabular}

McBane 2019 (ADAM-VTE)

\section{Study characteristics}

\begin{tabular}{ll}
\hline Methods & Randomized, open-label, invetigator-initiated, IV phase, multicenter, superiority trial. \\
\hline Participants & 300 patients from 28 center in the United States. \\
& Mean age 64 years; $48 \%$ men, $65 \%$ had metastatic disease, $36 \%$ had DVT at baseline, $40 \%$ had PE at \\
baseline, $6,5 \%$ had previous VTE. \\
Both solid and hematological malignancy. The most frequent solid tumours were: colorectal (about \\
16\%), lung (about $17 \%$ ) and pancreatic (about 16\%).
\end{tabular}

Interventions

Intervention: Apixaban $10 \mathrm{mg}$ twice daily for seven days followed by $5 \mathrm{mg}$ twice daily for six months.

Control: Dalteparin (200 IU/kg for one month followed by $150 \mathrm{IU} / \mathrm{kg}$ once daily) for six months.

Discontinued treatment: $20 / 150(13.3 \%)$ in the intervention arm and $37 / 150(24.7 \%)$

Duration of follow-up for the following outcomes: 6 months
- Major bleeding
- Clinically relevant non-major bleeding
- Any recurrence of: DVT, PE, fatal PE and arterial thromboembolism
- Mortality

Screening test for DVT/PE: survillance related imaging

Diagnostic test for DVT: duplex ultrasonography and venography and CT or MRI.

Diagnostic test for PE: CT, MR, pulmonary angiography or VQ imaging.

Notes

- Funded by a grant from Bristol-Myers Squibb and Pfizer Inc.

- Ethical approval: The protocol and its amendments were approved by the ethics committee at each trial center.

- Conflict of interest: The authors declare no competing financial interest.

- ITT: The intention-to-treat analysis defined included all the patients who had undergone randomization and received at least one dose of the assigned treatment.

\section{Risk of bias}


McBane 2019 (ADAM-VTE) (Continued)

\section{Bias Authors' judgement Support for judgement}

Random sequence genera- Low risk tion (selection bias)
Quote:"Randomization was performed using an established interactive Webbased system that is used for all clinical trials conducted through the Mayo Clinic Cancer Center and through the ACCRU infrastructure."

\begin{tabular}{lll}
\hline $\begin{array}{l}\text { Allocation concealment } \\
\text { (selection bias) }\end{array}$ & Low risk & Not reported \\
& & Comment: probably not concealed
\end{tabular}

Blinding of participants High risk Not reported.

and personnel (perfor-

Not reported.

mance bias)

All outcomes

Comment: probably not blinded; knowledge of the assigned intervention may have led to differential behaviors across intervention groups (e.g. differential dropout, differential cross-over to an alternative intervention or differential administration of cointerventions)

\begin{tabular}{|c|c|c|}
\hline Blinding of outcome as- & Low risk & Not reported. \\
\hline All outcomes & & $\begin{array}{l}\text { Comment: probably not blinded; knowledge of the assigned intervention may } \\
\text { not have impacted the assessment of the physiological outcomes (mortality, } \\
\text { DVT, PE, bleeding, etc.). }\end{array}$ \\
\hline
\end{tabular}

\begin{tabular}{|c|c|c|}
\hline $\begin{array}{l}\text { Incomplete outcome data } \\
\text { (attrition bias) } \\
\text { All outcomes }\end{array}$ & High risk & $\begin{array}{l}\text { Comment: judgment based on comparison in the intervention arm between } \\
\text { rate of participants with missing data }(20 / 150(13.3 \%)) \text { and event rate }(23 / 130 \\
(17.7 \%)) \text { for the outcome mortality. Similary for the control arm: rate of partici- } \\
\text { pants with missing data } 37 / 150(24.6 \%)) \text { and event rate } 15 / 113(13.2 \%))\end{array}$ \\
\hline
\end{tabular}

$\begin{array}{ll}\begin{array}{l}\text { Free of selective report- } \\ \text { ing? }\end{array} & \text { Low risk } \\ \end{array}$
ing? the results section.

Free of other bias? Low risk Quote:" Study not reported as stopped early for benefit".

\section{Meyer 2002 (CANTHANOX)}

\section{Study characteristics}

\begin{tabular}{ll}
\hline Methods & Randomized clinical trial \\
\hline Participants & $\begin{array}{l}\text { 146 participants with cancer (solid or hematologic; active or in remission but on treatment); with PE, } \\
\text { DVT, or both } \\
\text { Minimum age } 18 \text { years, minimum life expectancy } 3 \text { months, mean age } 65.5 \text { years; } 45 \% \text { men }\end{array}$ \\
\hline
\end{tabular}

Interventions

Intervention: enoxaparin $1.5 \mathrm{mg} / \mathrm{kg}$ daily $\times 3$ months

Control: enoxaparin $1.5 \mathrm{mg} / \mathrm{kg}$ daily $\times 4$ days followed by warfarin (target INR $2-3$ ) $\times 3$ months; $41 \%$ of time on target

The continuation and nature of anticoagulant treatment after 3 months were left to the attending physician.

Discontinued treatment: $4 / 71(5.6 \%)$ in the intervention arm and 4/75 (5.3\%) in the control arm 
Meyer 2002 (CANTHANOX) (Continued)

- Mortality

- Asymptomatic VTE

- Symptomatic and objectively confirmed recurrent VTE

- Major bleeding

- Minor bleeding

- Thrombocytopenia

Screening test for VTE: radiologic surveillance

Diagnostic test for DVT: venography or compression ultrasonography

Diagnostic test for PE: pulmonary angiography or ventilation/perfusion scanning

Notes

- Funding: Aventis, Assistance Publique, Hospitaux de Paris

- Ethical approval: "the ethics committee of Saint-Louis Hospital in Paris approved the study protocol."

- Conflict of interest: not reported

- ITT: "analysis was performed on an intention to treat basis."

\section{Risk of bias}

\begin{tabular}{|c|c|c|}
\hline Bias & Authors' judgement & Support for judgement \\
\hline $\begin{array}{l}\text { Random sequence genera- } \\
\text { tion (selection bias) }\end{array}$ & Low risk & Quote: "Treatment allocation was balanced at each center in blocks of 4." \\
\hline $\begin{array}{l}\text { Allocation concealment } \\
\text { (selection bias) }\end{array}$ & Low risk & Quote: "randomisation was performed using pre-sealed treatment boxes." \\
\hline $\begin{array}{l}\text { Blinding of participants } \\
\text { and personnel (perfor- } \\
\text { mance bias) } \\
\text { All outcomes }\end{array}$ & High risk & $\begin{array}{l}\text { Open-label study } \\
\text { Comment: definitely not blinded; knowledge of the assigned intervention may } \\
\text { have led to differential behaviors across intervention groups (e.g. differential } \\
\text { dropout, differential cross-over to an alternative intervention or differential } \\
\text { administration of cointerventions). }\end{array}$ \\
\hline $\begin{array}{l}\text { Blinding of outcome as- } \\
\text { sessment (detection bias) } \\
\text { All outcomes }\end{array}$ & Low risk & $\begin{array}{l}\text { Quote: "all potential outcome events were assessed by an independent adju- } \\
\text { dication committee whose members were unaware of the treatment assign- } \\
\text { ment." } \\
\text { Comment: definitely blinded }\end{array}$ \\
\hline
\end{tabular}

Incomplete outcome data Low risk

(attrition bias)

All outcomes

Comment: judgment based on comparison in the intervention arm between rate of participants with missing data $(4 / 71(5.6 \%))$ and event rate $(11.3 \%)$ for the outcome mortality. Similary for the control arm: rate of participants with missing data (4/75 (5.3\%)) and event rate (22.7\%)

\begin{tabular}{lll}
$\begin{array}{l}\text { Free of selective report- } \\
\text { ing? }\end{array}$ & Low risk & $\begin{array}{l}\text { Study not registered and no published protocol identified. All relevant out- } \\
\text { comes listed in the methods section were reported on in the results section. }\end{array}$ \\
\hline Free of other bias? & Low risk & Study not reported as stopped early for benefit \\
& & No other bias suspected
\end{tabular}


Prins 2014 (EINSTEIN)

\section{Study characteristics}

Methods Subgroup analysis of participants with active cancer in the EINSTEIN-DVT and EINSTEIN-PE phase 3 open-label multicenter trials

Participants

459 participants with active cancer, symptomatic DVT and PE enrolled from 314 centers in 38 countries

Median age $65-75$ years, $56 \%$ males, $22 \%$ had metastatic disease, $26 \%$ received chemotherapy

Interventions

Intervention: rivaroxaban $15 \mathrm{mg}$ twice daily for 21 days, followed by $20 \mathrm{mg}$ once daily for 3,6 or 12 months

Control: enoxaparin $1 \mathrm{mg} / \mathrm{kg}$ twice daily started within 48 hours after randomization and discontinued when the INR was $\geq 2$ for 2 days consecutively and the participant had received $\geq 5$ days and warfarin or acenocoumarol (adjusted to maintain INR 2-3) for 3, 6 or 12 months

Discontinued treatment: not reported for cancer subgroup

Outcomes Duration of follow-up was for the intended treatment period (3, 6 or 12 months) at 1 week, 2 weeks, 1 month and monthly thereafter for the following outcomes:

- All-cause mortality

- Symptomatic recurrent VTE

- Major bleeding

- Clinically relevant non-major bleeding (using validated measure of treatment satisfaction - the Anti-Clot Treatment Scale (ACTS))

Screening test for DVT/PE: not reported

Diagnostic test for DVT/PE: echo-doppler for DVT and spiral CT scan for PE

Notes

- The EINSTEIN-DVT and EINSTEIN-PE studies registered at ClinicalTrials.gov, numbers NCT00440193 and NCT00439777

- Funding: Bayer HealthCare Pharmaceuticals and Janssen Research \& Development

- Ethical approval: "The study protocols were reviewed and approved by the institutional review boards of each participating centre."

- Conflict of interest: More than one author reported financial COI

- ITT: "we did efficacy and mortality analyses on an intention-to-treat basis."

\section{Risk of bias}

\begin{tabular}{lll}
\hline Bias & Authors' judgement & Support for judgement \\
\hline $\begin{array}{l}\text { Random sequence genera- } \\
\text { tion (selection bias) }\end{array}$ & Low risk & $\begin{array}{l}\text { Quote: "Randomisation was done separately for participants with deep-vein } \\
\text { thrombosis and pulmonary embolism (with or without deep-vein thrombosis), } \\
\text { with a computerised voice-response system, and was stratified according to } \\
\text { country and the intended treatment duration (3, 6, or 12 months), as decided } \\
\text { locally before randomisation." }\end{array}$ \\
\hline $\begin{array}{l}\text { Allocation concealment } \\
\text { (selection bias) }\end{array}$ & Low risk & $\begin{array}{l}\text { Quote from protocol: "Allocation to treatment will be done centrally by inter- } \\
\text { active voice response system for Einstein-DVT and Einstein-PE, separately." }\end{array}$ \\
\hline
\end{tabular}


Prins 2014 (EINSTEIN) (Continued)
Blinding of participants
High risk
Open-label study and personnel (perfor- mance bias)
Comment: definitely not blinded; knowledge of the assigned intervention may
All outcomes have led to differential behaviors across intervention groups (e.g. differential dropout, differential cross-over to an alternative intervention or differential administration of cointerventions).

\begin{tabular}{|c|c|c|}
\hline $\begin{array}{l}\text { Blinding of outcome as- } \\
\text { sessment (detection bias) }\end{array}$ & Low risk & $\begin{array}{l}\text { Quote: "All suspected outcomes were classified by an independent blinded ad- } \\
\text { judication committee." }\end{array}$ \\
\hline All outcomes & & Comment: definitely blinded \\
\hline
\end{tabular}

Incomplete outcome data Low risk No information about follow-up in the cancer subgroup was reported.
(attrition bias)

All outcomes

\begin{tabular}{|c|c|c|}
\hline $\begin{array}{l}\text { Free of selective report- } \\
\text { ing? }\end{array}$ & Low risk & $\begin{array}{l}\text { Study registered and published protocol identified. All outcomes listed in the } \\
\text { methods section were reported on in the results section. }\end{array}$ \\
\hline
\end{tabular}

\begin{tabular}{ll}
\hline Free of other bias? & Low risk \\
& No other bias suspected \\
\hline
\end{tabular}

\section{Raskob 2016 (HOKUSAI)}

\section{Study characteristics}

\begin{tabular}{ll}
\hline Methods & $\begin{array}{l}\text { Subgroup analysis of participants with cancer or history of cancer in the HOKUSAI trial } \\
\text { Randomized, double-blind, double-dummy, multicenter trial }\end{array}$ \\
\hline Participants & $\begin{array}{l}208 \text { participants with active cancer at baseline from } 439 \text { centers in } 37 \text { countries (208 with active can- } \\
\text { cer prespecified categorization made by study physician at enrolment; } 162 \text { with active cancer post-hoc } \\
\text { classification) }\end{array}$ \\
& $\begin{array}{l}\text { Mean age } 66 \text { years, } 50 \% \text { male, } 6 \% \text { with metastatic disease, } 10 \% \text { receiving systemic cancer-drug thera- } \\
\text { py, excludes } 77 \text { participants with non-melanoma skin cancer }\end{array}$ \\
\hline
\end{tabular}

Interventions

All participants received initial therapy with open-label enoxaparin or UFH for $\geq 5$ days

Intervention: edoxaban $60 \mathrm{mg}$ once per day or $30 \mathrm{mg}$ once per day + dummy warfarin for $\geq 3$ months

Control: warfarin concurrently started with the study regimen of heparin (adjusted to maintain INR $2-3$ ) + dummy edoxaban for $\geq 3$ months. Enoxaparin was discontinued when the INR was $\geq 2$ for 2 days consecutively and the participant had received $\geq 5$ days of enoxaparin treatment

Initial therapy with open-label enoxaparin or UFH for $\geq 5$ days

Discontinued treatment: not reported for the active cancer subgroup

Duration of follow-up for the following outcomes: 12 months
- Mortality
- Adjudicated symptomatic recurrent VTE (defined as the composite of DVT or non-fatal or fatal PE)
- First occurrence of symptomatic recurrent VTE
- Major bleeding
- Clinically relevant non-major bleeding


Raskob 2016 (HOKUSAI) (Continued)

Screening test for DVT/PE: not reported

Diagnostic test for DVT/PE: not reported

Notes

- Study details obtained from original HOKUSAI methodology report published in Journal of Thrombosis and Haemostasis July 2013

- Study registered at ClinicalTrials.gov, number: NCT00986154

- Funding: Daiichi Sankyo

- Ethical approval: "The institutional review board at each centre approved the protocol."

- Conflict of interest: More than one author reported financial COI

- ITT: "use of a modified intention-to-treat analysis"

\section{Risk of bias}

\begin{tabular}{lll}
\hline Bias & Authors' judgement & Support for judgement \\
\hline $\begin{array}{l}\text { Random sequence genera- } \\
\text { tion (selection bias) }\end{array}$ & Low risk & $\begin{array}{l}\text { Quote: "local site study physician or study coordinator did the randomisation } \\
\text { using an interactive web-based system, with stratification according to the } \\
\text { qualifying diagnosis (deep-vein thrombosis or pulmonary embolism), pres- } \\
\text { ence or absence of temporary risk factors, and the dose of edoxaban." }\end{array}$ \\
\hline $\begin{array}{l}\text { Allocation concealment } \\
\text { (selection bias) }\end{array}$ & Low risk & $\begin{array}{l}\text { Quote: "The investigator provides this information to an interactive telephone } \\
\text { and web-based management system (IXRS; Almac, Yardley, PA, USA), which } \\
\text { randomly assigns the participant to the LMWH/edoxaban or standard therapy } \\
\text { group, and allocates the appropriate drug supply. The day of randomisation is } \\
\text { day } 1 \text { of the study." }\end{array}$ \\
\hline
\end{tabular}

\begin{tabular}{|c|c|c|}
\hline $\begin{array}{l}\text { Blinding of participants } \\
\text { and personnel (perfor- } \\
\text { mance bias) }\end{array}$ & Low risk & $\begin{array}{l}\text { Double-blind trial } \\
\text { Comment: definitely blinded }\end{array}$ \\
\hline
\end{tabular}

All outcomes

\begin{tabular}{|c|c|c|}
\hline $\begin{array}{l}\text { Blinding of outcome as- } \\
\text { sessment (detection bias) }\end{array}$ & Low risk & Quote: "central independent adjudication of all suspected outcomes" \\
\hline All outcomes & & Comment: definitely blinded \\
\hline
\end{tabular}

Incomplete outcome data Low risk No information about follow-up in the cancer subgroup was reported.
(attrition bias)

(attrition bias)

All outcomes

\begin{tabular}{|c|c|c|}
\hline $\begin{array}{l}\text { Free of selective report- } \\
\text { ing? }\end{array}$ & Low risk & $\begin{array}{l}\text { Study registered and published protocol identified. All outcomes reported in } \\
\text { the methods section were reported on in the results section. }\end{array}$ \\
\hline
\end{tabular}

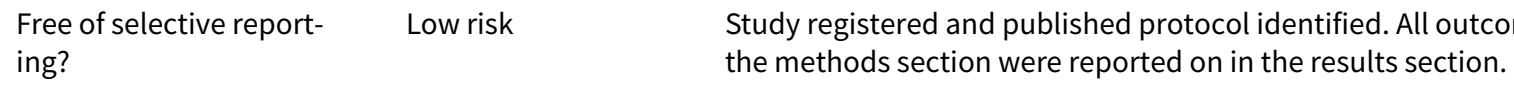

Free of other bias? Low risk Study not reported as stopped early for benefit

No other bias suspected

\section{Study characteristics}


Raskob 2018 (HOKUSAI) (Continued)

Participants 1050 people with active cancer from 114 centers in 13 countries with acute symptomatic or incidentally detected DVT or PE

Median age 64 years, $51.7 \%$ males, $53 \%$ had metastatic disease, $72.4 \%$ received cancer treatment within previous 4 weeks "anticancer drug therapy (cytotoxic, hormonal, targeted, or immunomodulatory), radiation therapy, surgery, or a combination of these therapies."

Interventions

All patients received initial therapy with LMWH for $\geq 5$ days. Duration of treatment: 6-12 months

Intervention: LMWH for $\geq 5$ days followed by oral edoxaban $60 \mathrm{mg}$ once daily

Control: dalteparin 200 IU per kilogram bodyweight SC once daily for 1 month followed by dalteparin 150 IU per kilogram once daily

Discontinued treatment: $48 / 525(9.1 \%)$ in the intervention arm and 34/525 (6.4\%)

\section{Outcomes} Duration of follow-up for the following outcomes: 12 months (on day 31 after randomization and months 3, 6, 9 and 12)

- Recurrent VTE

- Death from any cause

- Major bleeding

- Clinically relevant non-major bleeding

- Recurrent DVT

- Recurrent PE

- Event-free survival

Screening test for DVT/PE: "Incidental venous thromboembolism was defined as thromboembolism that was detected by means of imaging tests performed for reasons other than clinical suspicion of venous thromboembolism."

Diagnosis test for DVT/PE: "Appropriate diagnostic tests, laboratory tests, or both were required in people with suspected outcome events...aminotransferase and bilirubin levels."

Notes

- Study rationale and design of the HOKUSAI VTE-cancer study published in Journal of Thrombosis and Haemostasis August 2015.

- Funding: Daiichi Sankyo

- Ethical approval: "The institutional review board at each participating center approved the protocol." and "All the patients provided written informed consent."

- Conflict of interest: More than one author reported financial conflict of interest.

- ITT: "The analysis of the primary outcome was performed in the modified intention-to-treat population, which included all the patients who had undergone randomisation and received at least one dose of the assigned treatment."

\section{Risk of bias}

\begin{tabular}{lll}
\hline Bias & Authors' judgement & Support for judgement \\
\hline $\begin{array}{ll}\text { Random sequence genera- } \\
\text { tion (selection bias) }\end{array}$ & Low risk & $\begin{array}{l}\text { Quote: "Randomization was performed with the use of an interactive Web- } \\
\text { based system, with stratification according to whether risk factors for bleeding } \\
\text { were present and whether the patient met the criteria to receive a lower dose } \\
\text { of edoxaban." }\end{array}$ \\
\hline
\end{tabular}


Raskob 2018 (HOKUSAI) (Continued)

$\begin{array}{ll}\begin{array}{l}\text { Allocation concealment } \\ \text { (selection bias) }\end{array} & \begin{array}{l}\text { Quote: "Randomization was performed with the use of an interactive Web- } \\ \text { based system, with stratification according to whether risk factors for bleeding } \\ \text { were present and whether the patient met the criteria to receive a lower dose } \\ \text { of edoxaban." } \\ \text { Comment: probably concealed }\end{array}\end{array}$

\begin{tabular}{|c|c|c|}
\hline $\begin{array}{l}\text { Blinding of participants } \\
\text { and personnel (perfor- } \\
\text { mance bias) } \\
\text { All outcomes }\end{array}$ & High risk & $\begin{array}{l}\text { Quote: "Open label trial" } \\
\text { Comment: definitely not blinded; knowledge of the assigned intervention may } \\
\text { have led to differential behaviors across intervention groups (e.g. differential } \\
\text { dropout, differential cross-over to an alternative intervention or differential } \\
\text { administration of cointerventions). }\end{array}$ \\
\hline $\begin{array}{l}\text { Blinding of outcome as- } \\
\text { sessment (detection bias) } \\
\text { All outcomes }\end{array}$ & Low risk & $\begin{array}{l}\text { Quote: "all events were adjudicated by a committee whose members were un- } \\
\text { aware of the treatment assignments." } \\
\text { Comment: probably blinded; knowledge of the assigned intervention may not } \\
\text { have impacted the assessment of the physiological outcomes (mortality, DVT, } \\
\text { PE, bleeding, etc.). }\end{array}$ \\
\hline
\end{tabular}

Incomplete outcome data High risk

(attrition bias)

All outcomes

Comment: judgment based on comparison in the intervention arm between rate of participants with missing data (16/525 (3.04\%)) and event rate (34/509 $(6.7 \%))$ for the outcome recurrent VTE. Similary for the control arm: rate of participants with missing data (59/525 (11.2\%)) and event rate (46/507 (9.07\%)

\begin{tabular}{|c|c|c|}
\hline $\begin{array}{l}\text { Free of selective report- } \\
\text { ing? }\end{array}$ & Low risk & $\begin{array}{l}\text { Study registered and published protocol identified. All outcomes reported in } \\
\text { the methods section were reported on in the results section. }\end{array}$ \\
\hline
\end{tabular}

Free of other bias? Low risk

Study not reported as stopped early for benefit

No other bias suspected

Romera 2009

\section{Study characteristics}

\begin{tabular}{ll}
\hline Methods & Randomized trial \\
\hline Participants & 69 participants with cancer (study subgroup) and symptomatic proximal DVT \\
& Minimum age 18 years, mean age 61 years \\
\hline
\end{tabular}

Interventions

Intervention: tinzaparin SC fixed-dose $175 \mathrm{IU}$ anti-Xa per kg once daily for 6 months

Control: acenocoumarol $3 \mathrm{mg}$ orally, which was subsequently adjusted to achieve an INR of 2-3, tinzaparin was given until the INR reached $\geq 2$ on 2 consecutive measurements.

All participants received tinzaparin SC in a fixed dose of 175 IU anti-Xa per kg once daily

Discontinued treatment: not reported for cancer subgroup

Outcomes

Duration of follow-up for the following outcomes: 12 months

- VTE (no data available for other outcomes in participants with cancer) 
Romera 2009 (Continued)

Screening test for DVT/PE: not reported

Diagnostic test for DVT: duplex ultrasonography

Notes

- Funding: Hospital Universitari de Bellvitge, LEO Pharma

- Ethical approval: "The protocol was approved by the institutional review board at each centre and by the regulatory authorities."

- Conflict of interest: At least one author reported financial COI.

- ITT: not reported

\section{Risk of bias}

\begin{tabular}{lll}
\hline Bias & Authors' judgement & Support for judgement \\
\hline $\begin{array}{l}\text { Random sequence genera- } \\
\text { tion (selection bias) }\end{array}$ & Low risk & $\begin{array}{l}\text { Quote: "patients were randomised to either LMWH group SQ [subcutaneous] } \\
\text { or LMWH followed by acenocoumarol" } \\
\text { Comment: probably generated sequence randomly }\end{array}$ \\
\hline $\begin{array}{l}\text { Allocation concealment } \\
\text { (selection bias) }\end{array}$ & High risk & Not reported \\
\hline $\begin{array}{l}\text { Blinding of participants } \\
\text { and personnel (perfor- } \\
\text { mance bias) } \\
\begin{array}{l}\text { All outcomes } \\
\text { Comment: probably not concealed }\end{array} \\
\end{array} \quad$ High risk & $\begin{array}{l}\text { Open-label study } \\
\text { Comment: probably not blinded; knowledge of the assigned intervention may } \\
\text { have led to differential behaviors across intervention groups (e.g. differential } \\
\text { dropout, differential cross-over to an alternative intervention or differential } \\
\text { administration of cointerventions). }\end{array}$
\end{tabular}

Blinding of outcome as- Low risk sessment (detection bias)

All outcomes
Quote: "the ultrasonic evaluations were performed blindly;" "All objective diagnostic tests were interpreted by specialists who were not involved in the study."

Comment: definitely blinded

No information about follow-up in the cancer subgroup reported

Comment: assumed complete follow-up

Incomplete outcome data Low risk

(attrition bias)

All outcomes

Free of selective report- Low risk ing?

Low risk
Study was registered (NCT00689520). All relevant outcomes listed on the registration page and the methods section of the published manuscript were reported on in the results section.
Study not reported as stopped early for benefit

No other bias suspected

Schulman 2015 (RECOVER I-II)

\section{Study characteristics}

$\begin{array}{ll}\text { Methods } & \text { Subgroup analysis of participants with cancer at baseline, diagnosed with cancer during the study, or } \\ \text { history of cancer pooled from the RE-COVER and RE-COVER II trials }\end{array}$


Schulman 2015 (RECOVER I-II) (Continued)

Randomized, double-blind, double-dummy, multicenter trials

Participants 221 participants with active cancer at baseline and acute symptomatic proximal DVT or PE, from 228 clinical centers in 29 countries

Mean age 63.5 years, $61 \%$ male, $8 \%$ with metastatic cancer

Interventions

All participants received parenteral anticoagulant (UFH, LMWH or fondaparinux) until the INR or sham INR became $\geq 2$ for 2 consecutive days.

Intervention: dabigatran fixed-dose $150 \mathrm{mg}$ twice daily and warfarin-placebo

Control: dose-adjusted warfarin therapy, after initial parenteral anticoagulation and dabigatran-placebo

Cointervention: "initial treatment was with a parenteral anticoagulant (UFH, LMWH, or fondaparinux) until INR or sham INR became at least 2.0 for two consecutive days."

Discontinued treatment: not reported

Outcomes Duration of follow-up for the following outcomes: 6 months (assessed at 7 days and monthly thereafter)

- All-cause mortality

- Recurrent VTE

- Major bleeding

- Non-major clinically relevant bleeding

Screening test for DVT/PE: not reported

Diagnosis test for DVT/PE: not reported

Notes

- RECOVER and RECOVER II trials registered at ClinicalTrials.gov, numbers NCT00291330 and NCT00680186

- Funding Sources: Boehringer Ingelheim

- Ethical approval: "The institutional review board at each participating clinical centre approved the original studies"

- Conflict of interest: At least one author reported financial COI

- ITT: not reported

\section{Risk of bias}

\begin{tabular}{|c|c|c|}
\hline Bias & Authors' judgement & Support for judgement \\
\hline $\begin{array}{l}\text { Random sequence genera- } \\
\text { tion (selection bias) }\end{array}$ & Low risk & $\begin{array}{l}\text { Quote: "We used a computer generated randomisation scheme with variable } \\
\text { block sizes" (from main study RECOVER-I). }\end{array}$ \\
\hline & & $\begin{array}{l}\text { "Patients were randomised by use of an interactive voice response system and } \\
\text { a computer-generated randomisation scheme in blocks of } 4 \text { " (from main Studi } \\
\text { RECOVER-II). }\end{array}$ \\
\hline $\begin{array}{l}\text { Allocation concealment } \\
\text { (selection bias) }\end{array}$ & Low risk & $\begin{array}{l}\text { Quote: "If the patient was enrolled from the RE-COVER study or the RE-COVER } \\
\text { II study, a point-of-care coagulometer with encrypted INR results was used to } \\
\text { guide the transition so that the patients and investigators would remain un- } \\
\text { aware of the initial treatment." }\end{array}$ \\
\hline
\end{tabular}


Schulman 2015 (RECOVER I-II) (Continued)
Blinding of participants
Low risk
Double-blind trial and personnel (perfor-
Comment: definitely blinded
mance bias)

All outcomes

\begin{tabular}{|c|c|c|}
\hline $\begin{array}{l}\text { Blinding of outcome as- } \\
\text { sessment (detection bias) } \\
\text { All outcomes }\end{array}$ & Low risk & $\begin{array}{l}\text { Quote: "central adjudication committee" } \\
\text { Comment: definitely blinded }\end{array}$ \\
\hline
\end{tabular}

\begin{tabular}{lll}
\hline $\begin{array}{l}\text { Incomplete outcome data } \\
\text { (attrition bias) } \\
\text { All outcomes }\end{array}$ & Low risk & Complete follow-up (correspondence with author) \\
\hline $\begin{array}{l}\text { Free of selective report- } \\
\text { ing? }\end{array}$ & Low risk & $\begin{array}{l}\text { Study registered and published protocol identified. All outcomes reported in } \\
\text { the methods section were reported on in the results section. }\end{array}$ \\
\hline Free of other bias? & Low risk & $\begin{array}{l}\text { Study not reported as stopped early for benefit } \\
\text { No other bias suspected }\end{array}$ \\
\hline
\end{tabular}

van Doormaal 2010 (Van Gogh DVT trial)

\section{Study characteristics}

Methods

Post hoc analysis in the subgroup of participants with cancer included in the Van Gogh DVT clinical trial

randomized, open-label noninferiority trial

Participants

284 participants with active cancer having acute symptomatic and objectively confirmed DVT involving the popliteal, femoral, iliac veins or the trifurcation of the calf veins, without symptomatic PE

Quote: "no detailed information on cancer type and stage or co-medication was collected."

Interventions

Intervention: idraparinux $2.5 \mathrm{mg} \mathrm{SC}$ once-weekly $\times 3$ or 6 months according to the decision of treating physician

Control: standard treatment: tinzaparin, enoxaparin or intravenous heparin adjusted for the activated partial thromboplastin time ratio (ratio 1.5-2.5), followed by warfarin or acenocoumarol (INR 2-3), which was started within 24 hours after randomization.

Cointervention: not reported

Quote: "A total of $8 \%$ of all patients were randomised in the 3-month arm, and 92\% in the 6-month treatment arm."

Quote: "The duration of treatment was similar with a median of 183 days in both groups."

$75 \%$ of participants completed the study medication

Quote: "Of idraparinux recipients 48 patients (22\%) stopped the study medication before the end of the study compared to $56(28 \%)$ patients in the standard treatment arm."

Discontinued treatment: not reported for subgroup of patients with active cancer

Outcomes

Duration of follow-up for the following outcomes: 6-month treatment period plus additional 3-month follow-up period (median 183 days in both groups)

- All-cause mortality (follow-up at 6 and 9 months) 
van Doormaal 2010 (Van Gogh DVT trial) (Continued)

- Symptomatic objectively confirmed recurrent VTE: DVT (follow-up at 3 and 6 months), non-fatal or fatal PE (follow-up at 6 and 9 months)

- Clinically relevant major bleeding (follow-up at 3 and 6 months)

- Clinically relevant non-major bleeding (follow-up at 3 and 6 months)

Screening test for DVT/PE: not reported

Diagnostic test for DVT/PE: none reported in this manuscript, but available from Buller HR, New England Journal of Medicine 2007;357:1094-104

Diagnostic testing for PE: spiral computed tomography, pulmonary angiography

Diagnostic testing for DVT: ultrasonography, venography

Notes

- Funding: "The original trial was sponsored by Sanofi-Aventis. Their biostatisticians extracted the data of the present study."

- Ethical approval: "The protocols were approved by the institutional review board at each center."

- Conflict of interest: More than one author reported financial COI

- ITT: "The analyses were calculated in the intention to treat population."

\section{Risk of bias}

\begin{tabular}{lll}
\hline Bias & Authors' judgement & Support for judgement \\
\hline $\begin{array}{l}\text { Random sequence genera- } \\
\text { tion (selection bias) }\end{array}$ & Low risk & $\begin{array}{l}\text { Quote: "After giving written informed consent, patients were randomly as- } \\
\text { signed to receive either idraparinux or standard therapy with the use of a com- } \\
\text { puterized voice-response system" (from Buller HR, New England Journal of } \\
\text { Medicine 2007;357:1094-104). }\end{array}$ \\
\hline $\begin{array}{l}\text { Allocation concealment } \\
\text { (selection bias) }\end{array}$ & Low risk & $\begin{array}{l}\text { Quote: "After giving written informed consent, patients were randomly as- } \\
\text { signed to receive either idraparinux or standard therapy with the use of a com- } \\
\text { puterized voice-response system" (from Buller HR, New England Journal of } \\
\text { Medicine 2007;357:1094-104). }\end{array}$ \\
\hline
\end{tabular}

Blinding of participants High risk and personnel (performance bias)

All outcomes

\section{Open-label study}

Comment: definitely not blinded; knowledge of the assigned intervention may have led to differential behaviors across intervention groups (e.g. differential dropout, differential cross-over to an alternative intervention or differential administration of cointerventions).

\section{Blinding of outcome as- Low risk} sessment (detection bias) All outcomes
Quote: "All suspected outcomes were classified by an independent blinded adjudication committee."

Comment: definitely blinded; knowledge of the assigned intervention may not have impacted the assessment of the physiological outcomes (mortality, DVT, PE, bleeding, etc.).

\begin{tabular}{|c|c|c|}
\hline $\begin{array}{l}\text { Incomplete outcome data } \\
\text { (attrition bias) }\end{array}$ & Low risk & $\begin{array}{l}\text { No information about follow-up in the cancer subgroup reported } \\
\text { Comment: assumed complete follow-up }\end{array}$ \\
\hline
\end{tabular}

Free of selective report- Low risk ing?
Post-hoc analysis. Study registered and no published protocol identified. All relevant outcomes listed in the methods section were reported on in the results section. 
van Doormaal 2010 (Van Gogh DVT trial) (Continued)

$\begin{array}{ll}\text { Free of other bias? } & \text { Low risk } \\ & \text { No other bias suspected }\end{array}$

No other bias suspected

Young 2018 (SELECT-D)

\section{Study characteristics}

\begin{tabular}{ll}
\hline Methods & Prospective, randomized, open-label, multicenter pilot trial \\
\hline Participants & 406 people with active cancer at baseline with VTE from 58 centers across the UK \\
& $\begin{array}{l}\text { Mean age } 67 \text { years, } 53 \% \text { males, } 38 \% \text { early or locally advanced disease, } 59 \% \text { metastatic disease, } 57 \% \text { re- } \\
\text { ceiving chemotherapy, } 10 \% \text { receiving targeted therapy }\end{array}$ \\
\hline
\end{tabular}

Interventions Duration of treatment: 6 months

Intervention: rivaroxaban $15 \mathrm{mg}$ twice daily for 3 weeks then $20 \mathrm{mg}$ once daily, for 6 months in total

Control: dalteparin $200 \mathrm{IU} / \mathrm{kg}$ daily, month 1 and $150 \mathrm{lU} / \mathrm{kg}$, months 2-6

Discontinued treatment: not reported

Duration of follow-up for the following outcomes: 6 months
- Recurrent VTE
- Mortality
- Major bleeding
- Clinically relevant non-major bleeding
- Acceptability
- Health economics

Screening test for DVT/PE: compression ultrasound

Diagnosis test for DVT/PE: compression ultrasound

Notes

- Funding: Bayer PLC

- Ethical approval: not reported

- Conflict of interest: More than one author reported financial COI.

- ITT: not reported

\section{Risk of bias}

\begin{tabular}{lll}
\hline Bias & Authors' judgement & Support for judgement \\
\hline $\begin{array}{ll}\text { Random sequence genera- } \\
\text { tion (selection bias) }\end{array}$ & Low risk & $\begin{array}{l}\text { Quote: "Patients were randomly assigned centrally by telephoning Warwick } \\
\text { Clinical Trials Unit. Consenting patients were randomly assigned at a one-to- } \\
\text { one ratio using a computer-based minimization algorithm with..." }\end{array}$
\end{tabular}


Young 2018 (SELECT-D) (Continued)

$\begin{array}{ll}\begin{array}{l}\text { Allocation concealment } \\ \text { (selection bias) }\end{array} & \text { Quote: "Patients were randomly assigned centrally by telephoning Warwick } \\ & \text { Clinical Trials Unit. Consenting patients were randomly assigned at a one-to- } \\ & \text { one ratio using a computer-based minimization algorithm with..." }\end{array}$

$\begin{array}{ll}\begin{array}{l}\text { Blinding of participants } \\ \text { and personnel (perfor- }\end{array} & \text { High risk } \\ \begin{array}{l}\text { mance bias) } \\ \text { All outcomes }\end{array} & \begin{array}{l}\text { Quote: "Trial staff, participants, and investigators were not blinded to treat- } \\ \text { ment allocation" }\end{array} \\ & \begin{array}{l}\text { Comment: definitely not blinded; knowledge of the assigned intervention may } \\ \text { have led to differential behaviors across intervention groups (e.g. differential } \\ \text { dropout, differential cross-over to an alternative intervention or differential } \\ \text { administration of cointerventions). }\end{array}\end{array}$

\begin{tabular}{|c|c|c|}
\hline $\begin{array}{l}\text { Blinding of outcome as- } \\
\text { sessment (detection bias) }\end{array}$ & Low risk & $\begin{array}{l}\text { Quote: "Trial staff, participants, and investigators were not blinded to treat- } \\
\text { ment allocation" }\end{array}$ \\
\hline All outcomes & & $\begin{array}{l}\text { Comment: probably not blinded; however, knowledge of the assigned inter- } \\
\text { vention may not have impacted the assessment of the physiological outcomes } \\
\text { (mortality, DVT, PE, bleeding, etc.). }\end{array}$ \\
\hline
\end{tabular}

\begin{tabular}{lll}
\hline $\begin{array}{l}\text { Incomplete outcome data } \\
\text { (attrition bias) }\end{array}$ & Low risk & $\begin{array}{l}\text { Comment: judgment based on comparison in the intervention arm between } \\
\text { ratl outcomes }\end{array}$ \\
& $\begin{array}{l}(25.8 \%)) \text { for the outcome mortality. Similary for the control arm: rate of partici- } \\
\text { pants with missing data }(23 / 203(11.3 \%)) \text { and event rate }(56 / 180(31.1 \%)\end{array}$ \\
\hline $\begin{array}{l}\text { Free of selective report- } \\
\text { ing? }\end{array}$ & Low risk & $\begin{array}{l}\text { Study registered. All relevant outcomes listed in the methods section were re- } \\
\text { ported on in the results section }\end{array}$ \\
\hline
\end{tabular}

Free of other bias? Low risk Study not reported as stopped early for benefit

No other bias suspected

CT: computer tomography; COI: conflict of interest; DOAC: direct oral anticoagulant; DVT: deep venous thrombosis; ECOG: Eastern Cooperative Oncology Group; HRQoL: health-related quality of life; INR: international normalized ratio; ITT: intention to treat; IU: international unit; LMWH: low molecular weight heparin; MPD: missing participants data; PE: pulmonary embolism; SC: subcutaneous; U: unit; UFH: unfractionated heparin; VKA: vitamin K antagonist; VTE: venous thromboembolism.

\section{Characteristics of excluded studies [ordered by study ID]}

\begin{tabular}{ll}
\hline Study & Reason for exclusion \\
\hline Agnelli 2005 & Not population of interest (surgical setting) \\
\hline Alikhan 2003 (MEDENOX) & Not population of interest (people with cancer without VTE) \\
\hline Auer 2011 & Not population of interest (people with cancer without VTE) \\
\hline Cohen 2006 & Not population of interest (people with cancer without VTE) \\
\hline Cohen 2007 (PREVENT) & Not population of interest (people with cancer without VTE) \\
\hline Couban 2005 & Not population of interest (people with cancer with CVC without VTE) \\
\hline Eriksson 2005 & Not population of interest (people without cancer) \\
\hline Farred 2004 & Not design of interest (review) \\
\hline
\end{tabular}




\begin{tabular}{|c|c|}
\hline Study & Reason for exclusion \\
\hline Ferretti 2005 & Not design of interest (review) \\
\hline Ferretti 2006 & Not design of interest (review) \\
\hline Fiessinger 2005 & Outcome data for cancer subgroup not reported \\
\hline Haas 2011 & Not population of interest (people with cancer without VTE) \\
\hline Hata 2016 & Not population of interest (people with cancer without VTE) \\
\hline Hull 2007 & Outcome data for cancer subgroup not reported \\
\hline Hull 2009 & Outcome data for cancer subgroup not reported \\
\hline Hyers 2005 & Not design of interest (review) \\
\hline Kakkar 2003 & Not population of interest (patients without cancer) \\
\hline Kakkar 2010 (CANBESURE) & Not population of interest (people with cancer without VTE) \\
\hline Kakkar 2014 (SAVE-ABDO) & Not population of interest (people with cancer without VTE) \\
\hline Khorana 2017 (PHACS) & Not population of interest (people with cancer without VTE) \\
\hline King 2005 & Not design of interest (retrospective) \\
\hline Kovacs 2005 & Not design of interest (observational) \\
\hline Kucher 2005 & Outcome data for cancer subgroup not reported \\
\hline Larocca 2012 & Not population of interest (people with cancer without VTE) \\
\hline Lee 2005 & Not design of interest (review) \\
\hline Lee 2006 & Not design of interest (review) \\
\hline Levine 2003 & Not design of interest (review) \\
\hline Macbeth 2016 (FRAGMATIC) & Not population of interest (people with cancer without VTE) \\
\hline Massicotte 2003 & Outcome data for cancer subgroup not reported \\
\hline Murakami 2002 & Not population of interest (people with cancer without VTE) \\
\hline Nagata 2015 & Not population of interest (people with cancer without VTE) \\
\hline Palumbo 2011 & Not population of interest (people with cancer without VTE) \\
\hline Pelzer 2015 (CONKO-004) & Not population of interest (people with cancer without VTE) \\
\hline Pérez-de-Llano 2010 & Outcome data for cancer subgroup not reported \\
\hline Sakon 2010 & Not population of interest (people with cancer without VTE) \\
\hline Schulman 2003 & Not intervention of interest (extended treatment) \\
\hline
\end{tabular}




\begin{tabular}{ll}
\hline Study & Reason for exclusion \\
\hline Schulman 2006 & Not population of interest (none of participants had cancer) \\
\hline Schulman 2013 (RE-MEDY) & Not intervention of interest (extended treatment) \\
\hline Siragusa 2010 & Not intervention of interest: different duration of interventional drugs \\
\hline Song 2014 & Not population of interest (people with cancer without VTE) \\
\hline Suarez Alvarez 2003 & Not design of interest (not an RCT) \\
\hline Vedovati 2014 & Not population of interest (people with cancer without VTE) \\
\hline Veiga 2000 & Outcome data for cancer subgroup not reported \\
\hline Verso 2008 & Not population of interest (people with cancer without VTE) \\
\hline Zheng 2014 & Not population of interest (people with cancer without VTE) \\
\hline Zwicker 2013 (MICROTEC) & Not population of interest (people with cancer without VTE) \\
\hline
\end{tabular}

DOAC: direct oral anticoagulant; LMWH: low molecular weight heparin; RCT: randomized controlled trial; VTE: venous thromboembolism.

Characteristics of ongoing studies [ordered by study ID]

Kamphuisen 2010 (Longheva)

Study name PO-67 Long-term treatment for cancer patients with deep vein thrombosis or pulmonary embolism - a randomised controlled trial

\begin{tabular}{ll}
\hline Methods & Multicenter, multinational, randomized, open-label trial \\
\hline Participants & $\begin{array}{l}\text { Participants with malignancy (all types, solid and hematologic) who had received 6-12 months of } \\
\text { anticoagulation for VTE and had an indication for continuing anticoagulation }\end{array}$ \\
\hline Interventions & $\begin{array}{l}\text { Intervention: weight-adjusted scheme of LMWH for } 6 \text { additional months, } 65-75 \% \text { of full therapeutic } \\
\text { dose }\end{array}$ \\
Control: VKA for 6 additional months
\end{tabular}

\begin{tabular}{ll}
\hline Outcomes & $\begin{array}{l}\text { Symptomatic recurrent VTE (DVT and PE), all clinically relevant bleeding (i.e. major bleeding and } \\
\text { other clinically relevant non-major bleeding), all-cause mortality }\end{array}$ \\
\hline Starting date & August 2010 \\
\hline Contact information & Professor Pieter W Kamphuisen, telephone: 0031503612943, email: p.w.kamphuisen@umcg.nl \\
\hline Notes & Status as of May 2021: Terminated (Due to slow inclusion of patients) \\
& Funding: University Medical Center Groningen \\
NCT: NCT01164046
\end{tabular}


Karatas 2015

\begin{tabular}{|c|c|}
\hline Study name & Rivaroxaban in the treatment of venous thromboembolism (VTE) in cancer patients \\
\hline Methods & Randomized open-label phase III trial \\
\hline Participants & Aged $\geq 18$ years with active malignancy and newly diagnosed and objectively confirmed acute VTE \\
\hline Interventions & $\begin{array}{l}\text { Drug: rivaroxaban } 15 \mathrm{mg} \text { twice daily for } 21 \text { days, followed by } 20 \mathrm{mg} \text { once daily over } 3 \text { months } \\
\text { Drug: LMWH in therapeutic dosage ( } 1-2 \times \text { daily SC) according to standards of the individual study } \\
\text { center, using licensed dosages }\end{array}$ \\
\hline Outcomes & $\begin{array}{l}\text { Primary outcome: participant-reported treatment satisfaction (convenience) with rivaroxaban in } \\
\text { the treatment of acute VTE in people with cancer in comparison with the standard treatment with } \\
\text { LMWH } \\
\text { Secondary outcome: rate of VTE }\end{array}$ \\
\hline Starting date & March 2016 \\
\hline Contact information & Dr Aysun Karatas, email: aysun.karatas@aio-studien-ggmbh.de \\
\hline Notes & Status as of May 2021: Terminated (Recruitment was not as expected). \\
\hline & $\begin{array}{l}\text { Funding: AIO-Studien-gGmbH } \\
\text { NCT02583191 }\end{array}$ \\
\hline
\end{tabular}

\section{Meyer 2016 (CASTA-DIVA)}

\begin{tabular}{|c|c|}
\hline Study name & Cancer associated thrombosis, a pilot treatment study using rivaroxaban (CASTA-DIVA) \\
\hline Methods & Randomized, open-label trial \\
\hline Participants & People with cancer aged > 18 years with objectively confirmed symptomatic VTE \\
\hline \multirow[t]{2}{*}{ Interventions } & $\begin{array}{l}\text { Intervention 1: dalteparin } 200 \mathrm{IU} / \mathrm{kg} \text { SC once daily for } 1 \text { month followed by } 150 \mathrm{IU} / \mathrm{kg} \text { SC once daily } \\
\text { for } 2 \text { months }\end{array}$ \\
\hline & $\begin{array}{l}\text { Intervention 2: rivaroxaban } 15 \mathrm{mg} \text { orally twice daily for } 3 \text { weeks followed by } 20 \mathrm{mg} \text { once daily for } 9 \\
\text { weeks }\end{array}$ \\
\hline \multirow[t]{2}{*}{ Outcomes } & Primary outcome: symptomatic DVT, PE at 3 months \\
\hline & $\begin{array}{l}\text { Secondary outcome: major and clinically significant bleedings during the 3-month treatment peri- } \\
\text { od }\end{array}$ \\
\hline Starting date & September 2016 \\
\hline Contact information & Guy Meyer, MD, email: guy.meyer@aphp.fr \\
\hline \multirow[t]{3}{*}{ Notes } & Status as of May 2021: completed \\
\hline & Funding: Assistance Publique - Hôpitaux de Paris \\
\hline & NCT02746185 \\
\hline
\end{tabular}




\begin{tabular}{|c|c|}
\hline Study name & $\begin{array}{l}\text { A randomized phase II study to compare the safety and efficacy of dalteparin vs. rivaroxaban for } \\
\text { cancer-associated venous thromboembolism (PRIORITY) }\end{array}$ \\
\hline Methods & Multicenter, randomized, open-label phase II trial \\
\hline Participants & $\begin{array}{l}\text { Aged } \geq 18 \text { years with confirmed locally advanced unresectable or metastatic active cancer and } \\
\text { newly diagnosed DVT or PE }\end{array}$ \\
\hline \multirow[t]{2}{*}{ Interventions } & $\begin{array}{l}\text { Intervention 1: dalteparin } 200 \mathrm{IU} / \mathrm{kg} \text { SC once daily for } 4 \text { weeks followed by } 150 \mathrm{IU} / \mathrm{kg} \text { once daily for } \\
20 \text { weeks }\end{array}$ \\
\hline & $\begin{array}{l}\text { Intervention 2: rivaroxaban } 15 \mathrm{mg} \text { orally twice daily for } 3 \text { weeks followed by } 20 \mathrm{mg} \text { once daily for } 21 \\
\text { weeks }\end{array}$ \\
\hline \multirow[t]{2}{*}{ Outcomes } & Primary outcome: rate of clinical relevant bleeding \\
\hline & Secondary outcome: total event of bleeding, time to event of bleeding, recurrent VTE \\
\hline Starting date & May 2017 \\
\hline Contact information & Sook Ryun Park, MD, PhD, email: srpark@amc.seoul.kr \\
\hline \multirow[t]{3}{*}{ Notes } & Status as of May 2021: recruiting \\
\hline & Funding: Asan Medical Center \\
\hline & NCT03139487 \\
\hline
\end{tabular}

\section{Schrag 2016 (CANVAS)}

\begin{tabular}{ll}
\hline Study name & Direct oral anticoagulants (DOACS) versus LMWH \pm warfarin for VTE in cancer (CANVAS) \\
\hline Methods & Randomized, open-label trial \\
\hline Participants & $\begin{array}{l}\text { Aged } \geq 21 \text { years with solid tumor cancer, lymphoma or myeloma, diagnosed with VTE <30 days pri- } \\
\text { or to study enrolment }\end{array}$ \\
\hline Interventions & Intervention 1: DOAC \\
\hline Intervention 2: LMWH with or without transition to warfarin \\
\hline Primary outcome: cumulative VTE recurrence \\
Secondary outcome: major bleeding, burden of anticoagulation therapy, mortality \\
\hline December 2016 \\
\hline Deborah Schrag, MD MPH, telephone: 617-582-8301, email: deb_schrag@dfci.harvard.edu \\
\hline Status as of May 2021: compteted \\
Funding: Patient-Centered Outcomes Research Institute (PCORI) \\
NCT NCT02744092
\end{tabular}


DOAC: direct oral anticoagulant; DVT: deep venous thrombosis; LMWH: low molecular weight heparin; PE: pulmonary embolism; SC: subcutaneous; VTE: venous thromboembolism.

\section{DATA AND ANALYSES}

Comparison 1. Low molecular weight heparins (LMWH) versus vitamin K antagonists (VKA)

\begin{tabular}{|c|c|c|c|c|}
\hline Outcome or subgroup title & No. of studies & $\begin{array}{l}\text { No. of partici- } \\
\text { pants }\end{array}$ & Statistical method & Effect size \\
\hline $\begin{array}{l}\text { 1.1 All-cause mortality (up to } 6 \text { months) } \\
\text { (main analysis - active cancer) }\end{array}$ & 4 & 1712 & $\begin{array}{l}\text { Risk Ratio (M-H, Ran- } \\
\text { dom, } 95 \% \mathrm{Cl})\end{array}$ & $0.99[0.88,1.12]$ \\
\hline 1.2 All-cause mortality (time-to-event) & 2 & 810 & HR (IV, Random, 95\% Cl) & $0.94[0.74,1.20]$ \\
\hline $\begin{array}{l}\text { 1.3 Recurrent venous thromboembolism (up } \\
\text { to } 6 \text { months) (main analysis - active cancer) }\end{array}$ & 4 & 1712 & $\begin{array}{l}\text { Risk Ratio (M-H, Ran- } \\
\text { dom, } 95 \% \mathrm{Cl})\end{array}$ & $0.59[0.44,0.80]$ \\
\hline $\begin{array}{l}\text { 1.4 Recurrent venous thromboembolism } \\
\text { (time-to-event) }\end{array}$ & 2 & 810 & HR (IV, Random, 95\% Cl) & $0.49[0.31,0.78]$ \\
\hline $\begin{array}{l}1.5 \text { Major bleeding (up to } 6 \text { months) (main } \\
\text { analysis - active cancer) }\end{array}$ & 4 & 1712 & $\begin{array}{l}\text { Risk Ratio (M-H, Ran- } \\
\text { dom, } 95 \% \mathrm{Cl})\end{array}$ & $1.09[0.55,2.12]$ \\
\hline $\begin{array}{l}\text { 1.6 Minor bleeding (up to } 6 \text { months) (main } \\
\text { analysis - active cancer) }\end{array}$ & 4 & 1712 & $\begin{array}{l}\text { Risk Ratio (M-H, Ran- } \\
\text { dom, } 95 \% \mathrm{Cl})\end{array}$ & $0.78[0.47,1.27]$ \\
\hline $\begin{array}{l}\text { 1.7 Thrombocytopenia (up to } 6 \text { months) } \\
\text { (main analysis- active cancer) }\end{array}$ & 1 & 138 & $\begin{array}{l}\text { Risk Ratio (M-H, Ran- } \\
\text { dom, } 95 \% \mathrm{Cl})\end{array}$ & $0.94[0.52,1.69]$ \\
\hline
\end{tabular}

Analysis 1.1. Comparison 1: Low molecular weight heparins (LMWH) versus vitamin K antagonists (VKA), Outcome 1: All-cause mortality (up to 6 months) (main analysis - active cancer)

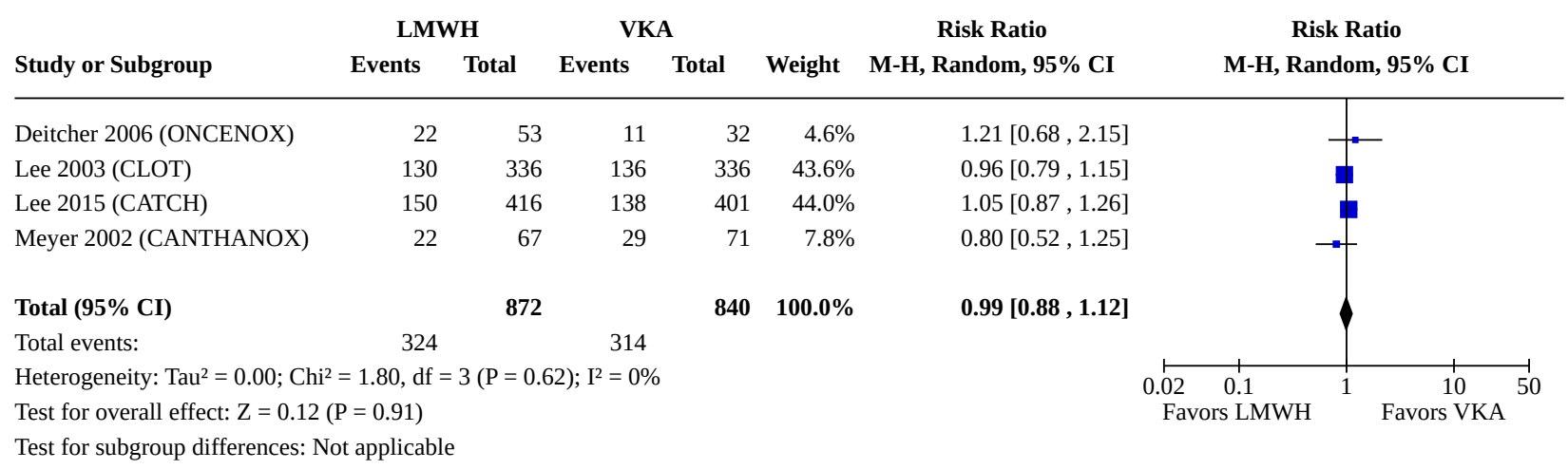


Analysis 1.2. Comparison 1: Low molecular weight heparins (LMWH) versus vitamin K antagonists (VKA), Outcome 2: All-cause mortality (time-to-event)

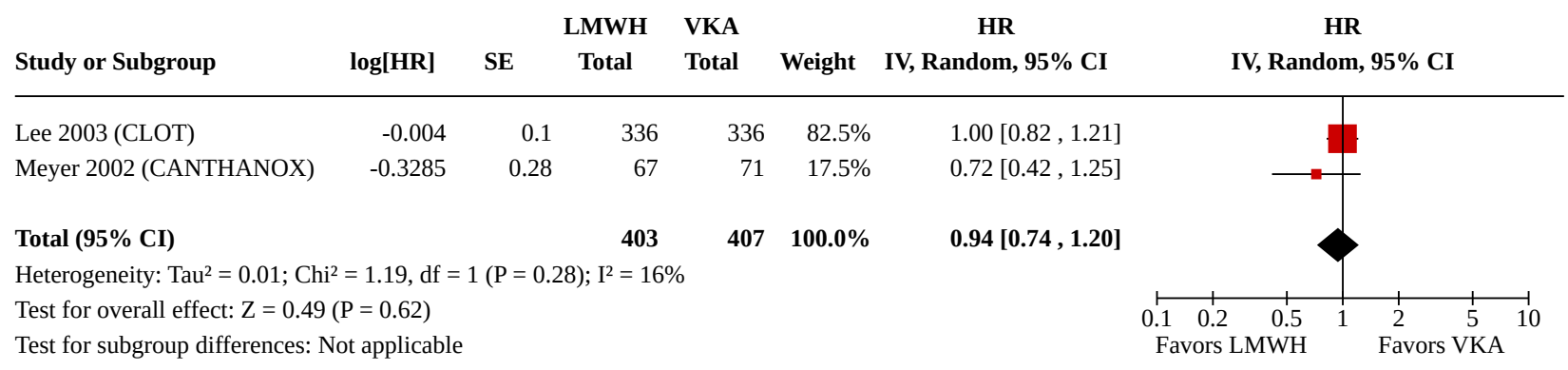

Analysis 1.3. Comparison 1: Low molecular weight heparins (LMWH) versus vitamin K antagonists (VKA), Outcome 3: Recurrent venous thromboembolism (up to 6 months) (main analysis - active cancer)

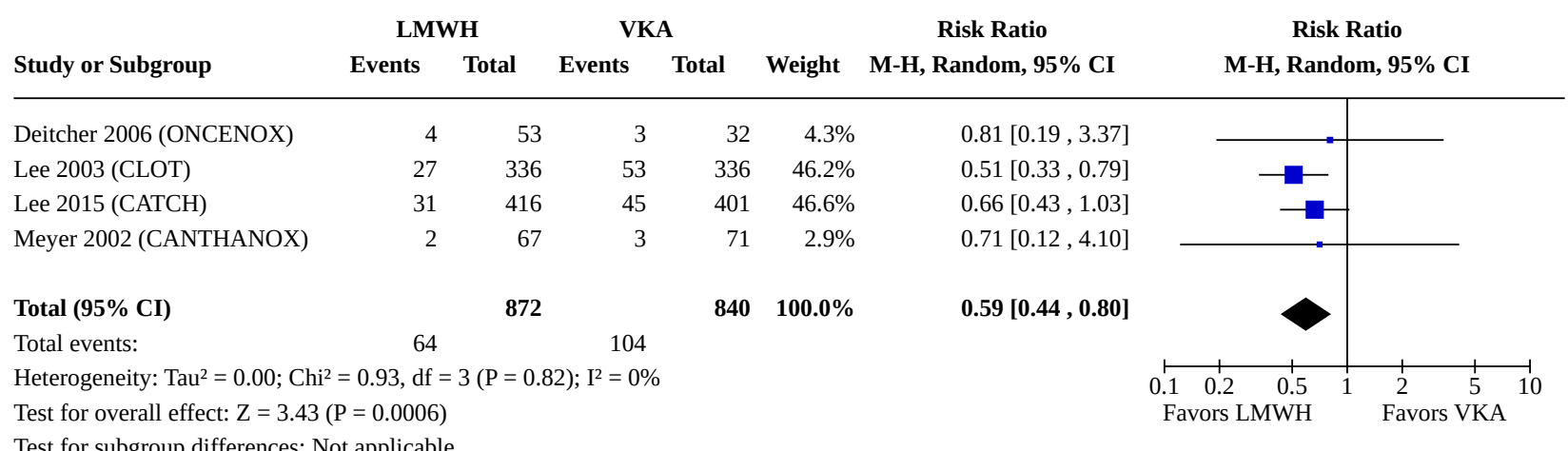

Analysis 1.4. Comparison 1: Low molecular weight heparins (LMWH) versus vitamin K antagonists (VKA), Outcome 4: Recurrent venous thromboembolism (time-to-event)

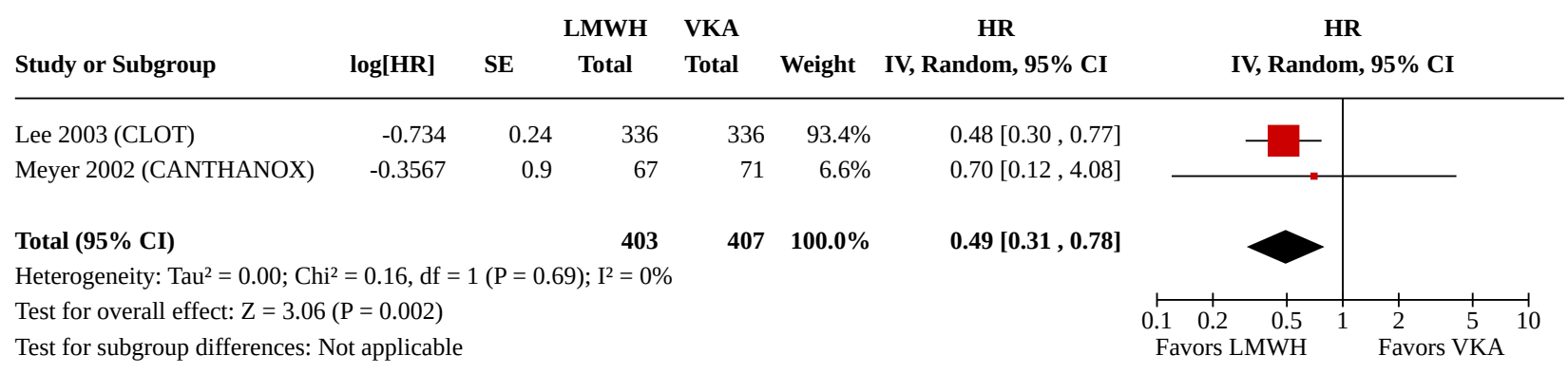


Analysis 1.5. Comparison 1: Low molecular weight heparins (LMWH) versus vitamin $K$ antagonists (VKA), Outcome 5: Major bleeding (up to 6 months) (main analysis - active cancer)

\begin{tabular}{|c|c|c|c|c|c|c|c|}
\hline & LM & & & & & Risk Ratio & Risk Ratio \\
\hline Study or Subgroup & Events & Total & Events & Total & Weight & M-H, Random, 95\% CI & M-H, Random, 95\% C \\
\hline
\end{tabular}

\begin{tabular}{|c|c|c|c|c|c|c|}
\hline Deitcher 2006 (ONCENOX) & 6 & 53 & 1 & 32 & $8.9 \%$ & $3.62[0.46,28.74]$ \\
\hline Lee 2003 (CLOT) & 19 & 336 & 12 & 336 & $34.7 \%$ & $1.58[0.78,3.21]$ \\
\hline Lee 2015 (CATCH) & 12 & 416 & 11 & 401 & $31.1 \%$ & $1.05[0.47,2.36]$ \\
\hline Meyer 2002 (CANTHANOX) & 5 & 67 & 12 & 71 & $25.4 \%$ & $0.44[0.16,1.19]$ \\
\hline Total $(95 \% \mathrm{CI})$ & & 872 & & 840 & $100.0 \%$ & $1.09[0.55,2.12]$ \\
\hline
\end{tabular}

Total events: $\quad 42 \quad 36$

Heterogeneity: $\mathrm{Tau}^{2}=0.21 ; \mathrm{Chi}^{2}=5.59, \mathrm{df}=3(\mathrm{P}=0.13) ; \mathrm{I}^{2}=46 \%$

Test for overall effect: $\mathrm{Z}=0.24(\mathrm{P}=0.81)$

Test for subgroup differences: Not applicable

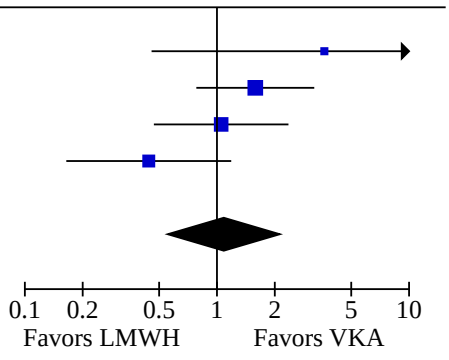

Analysis 1.6. Comparison 1: Low molecular weight heparins (LMWH) versus vitamin $K$ antagonists (VKA), Outcome 6: Minor bleeding (up to 6 months) (main analysis - active cancer)

\begin{tabular}{|c|c|c|c|c|c|c|c|}
\hline & $\mathbf{L M}$ & & VF & & & Risk Ratio & Risk Ratio \\
\hline Study or Subgroup & Events & Total & Events & Total & Weight & M-H, Random, 95\% CI & M-H, Random, 95\% CI \\
\hline
\end{tabular}

\begin{tabular}{|c|c|c|c|c|c|c|}
\hline Deitcher 2006 (ONCENOX) & 39 & 53 & 17 & 32 & $29.2 \%$ & $1.39[0.96,1.99]$ \\
\hline Lee 2003 (CLOT) & 28 & 336 & 51 & 336 & $27.3 \%$ & $0.55[0.36,0.85]$ \\
\hline Lee 2015 (CATCH) & 49 & 416 & 69 & 401 & $29.8 \%$ & $0.68[0.49,0.96]$ \\
\hline Meyer 2002 (CANTHANOX) & 5 & 67 & 9 & 71 & $13.7 \%$ & $0.59[0.21,1.67]$ \\
\hline Total (95\% CI) & & 872 & & 840 & $100.0 \%$ & $0.78[0.47,1.27]$ \\
\hline
\end{tabular}

Total

$121 \quad 146$

Heterogeneity: $\mathrm{Tau}^{2}=0.19 ; \mathrm{Chi}^{2}=13.85, \mathrm{df}=3(\mathrm{P}=0.003) ; \mathrm{I}^{2}=78 \%$

Test for overall effect: $\mathrm{Z}=1.00(\mathrm{P}=0.32)$

Test for subgroup differences: Not applicable

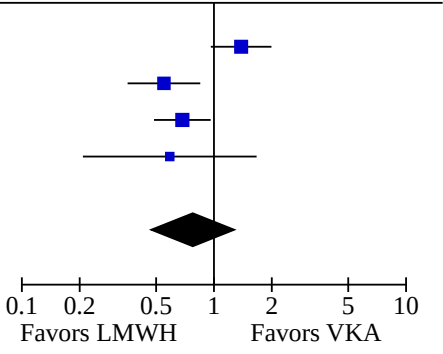

Analysis 1.7. Comparison 1: Low molecular weight heparins (LMWH) versus vitamin $K$ antagonists (VKA), Outcome 7: Thrombocytopenia (up to 6 months) (main analysis- active cancer)

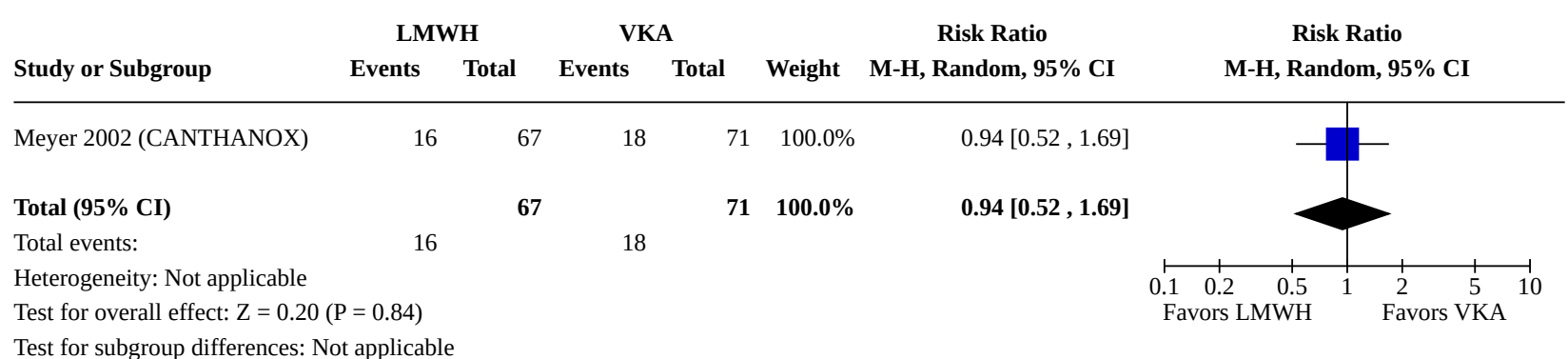

Comparison 2. Direct oral anticoagulants (DOAC) versus vitamin $\mathrm{K}$ antagonists (VKA)

\begin{tabular}{lllll}
\hline Outcome or subgroup title & No. of studies & $\begin{array}{l}\text { No. of partici- } \\
\text { pants }\end{array}$ & Statistical method & Effect size \\
\hline 2.1 All-cause mortality (6-12 months) & 4 & 1060 & $\begin{array}{l}\text { Risk Ratio (M-H, Random, } \\
95 \% \mathrm{Cl})\end{array}$ & $0.94[0.72,1.23]$ \\
\hline
\end{tabular}




\begin{tabular}{lllll}
\hline Outcome or subgroup title & No. of studies & $\begin{array}{l}\text { No. of partici- } \\
\text { pants }\end{array}$ & Statistical method & Effect size \\
\hline $\begin{array}{l}\text { 2.2 Recurrent venous thromboem- } \\
\text { bolism (6-12 months) }\end{array}$ & 4 & 1050 & $\begin{array}{l}\text { Risk Ratio (M-H, Random, } \\
95 \% \mathrm{Cl})\end{array}$ & $0.63[0.34,1.15]$ \\
\hline 2.3 Major bleeding (6-12 months) & 4 & 1055 & $\begin{array}{l}\text { Risk Ratio (M-H, Random, } \\
95 \% \mathrm{Cl})\end{array}$ & $0.77[0.39,1.53]$ \\
\hline 2.4 Minor bleeding (6-12 months) & 4 & 1055 & $\begin{array}{l}\text { Risk Ratio (M-H, Random, } \\
95 \% \mathrm{Cl})\end{array}$ & 0.83 [0.57, 1.23] \\
\hline
\end{tabular}

Analysis 2.1. Comparison 2: Direct oral anticoagulants (DOAC) versus vitamin K antagonists (VKA), Outcome 1: All-cause mortality (6-12 months)

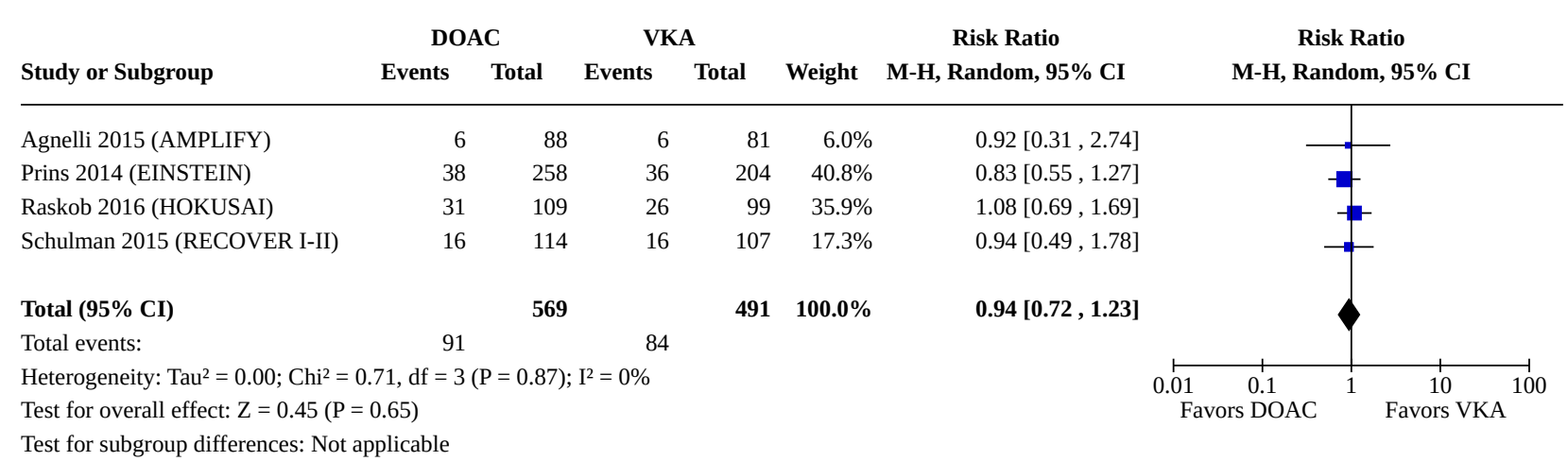

Analysis 2.2. Comparison 2: Direct oral anticoagulants (DOAC) versus vitamin $K$ antagonists (VKA), Outcome 2: Recurrent venous thromboembolism (6-12 months)

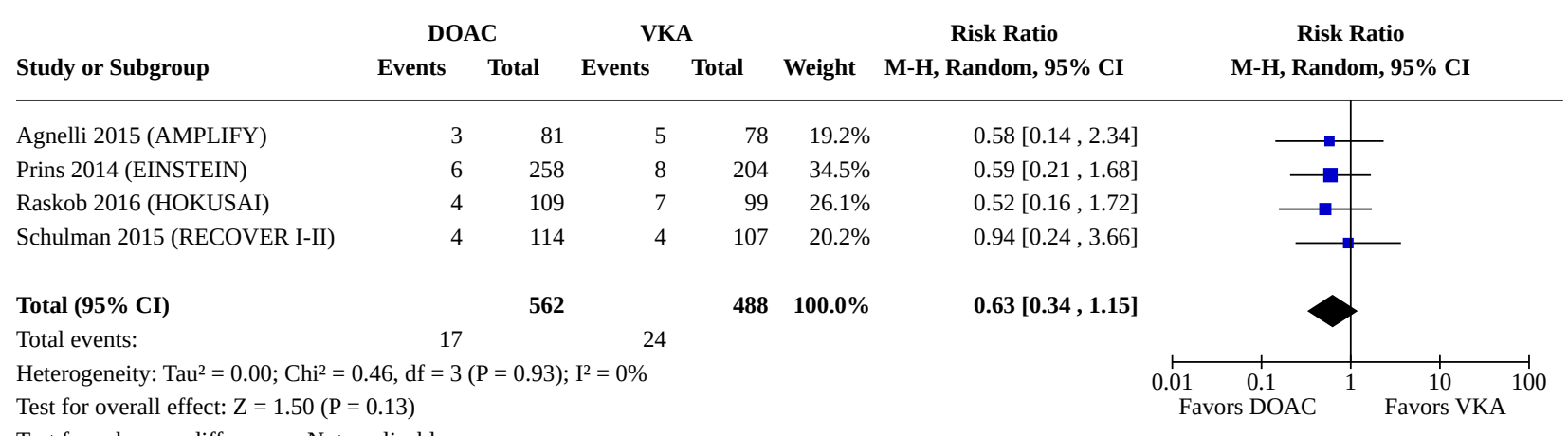


Analysis 2.3. Comparison 2: Direct oral anticoagulants (DOAC) versus vitamin K antagonists (VKA), Outcome 3: Major bleeding (6-12 months)

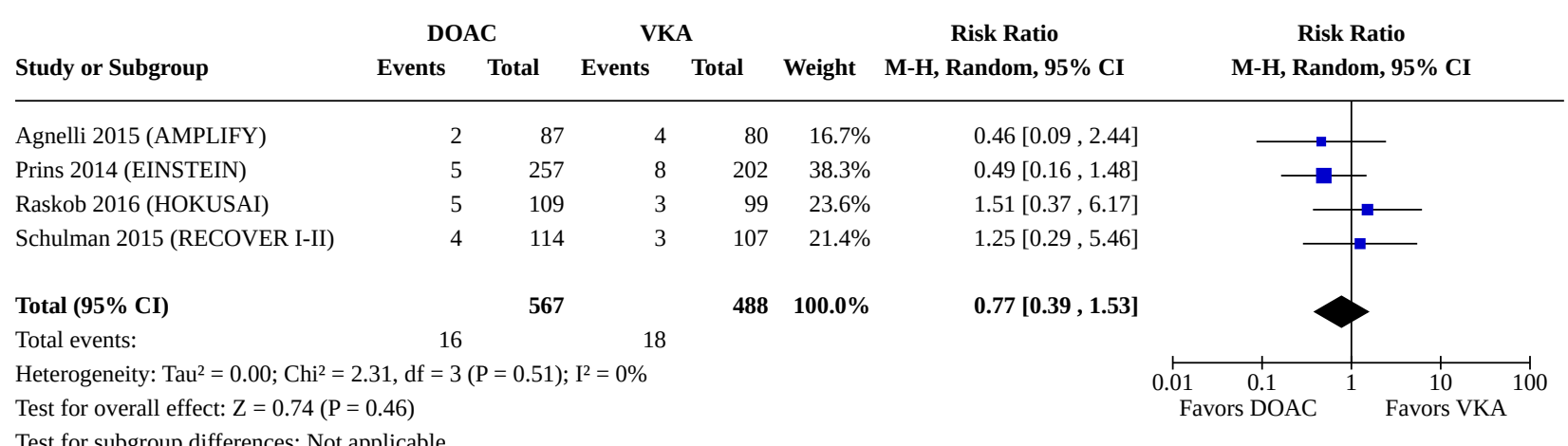

Analysis 2.4. Comparison 2: Direct oral anticoagulants (DOAC) versus vitamin $\mathrm{K}$ antagonists (VKA), Outcome 4: Minor bleeding (6-12 months)

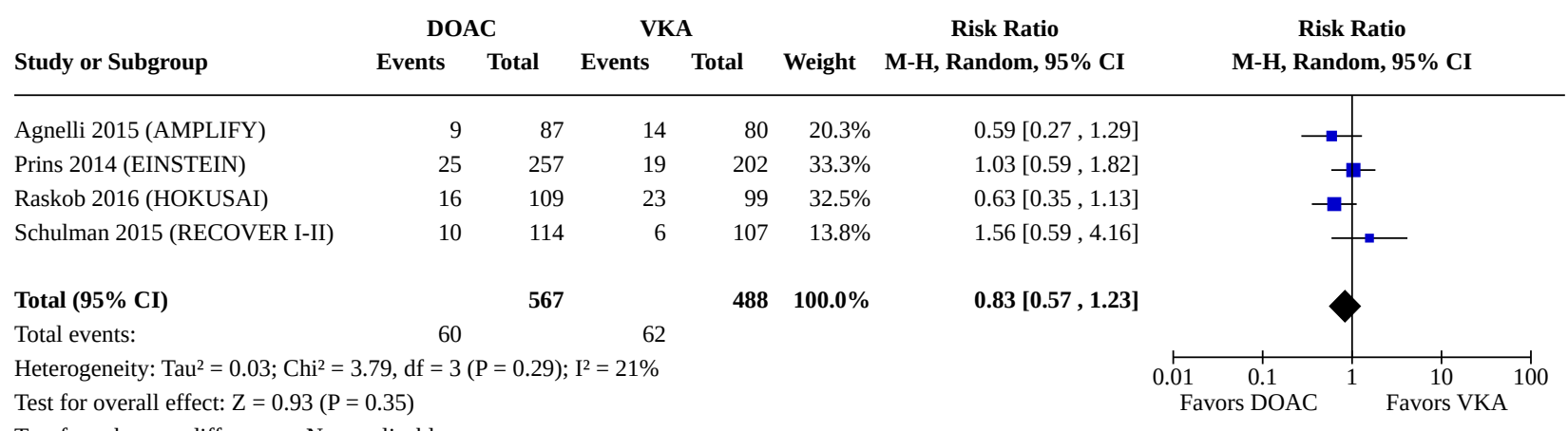

\section{Comparison 3. Direct oral anticoagulants (DOAC) versus low molecular weight heparins (LMWH)}

\begin{tabular}{lllll}
\hline Outcome or subgroup title & No. of studies & $\begin{array}{l}\text { No. of partici- } \\
\text { pants }\end{array}$ & Statistical method & Effect size \\
\hline 3.1 All-cause mortality (6 months) & 5 & 2854 & $\begin{array}{l}\text { Risk Ratio (M-H, Random, 95\% } \\
\text { Cl) }\end{array}$ & $0.97[0.83,1.14]$ \\
\hline 3.2 Recurrent VTE (6 months) & 5 & 2854 & $\begin{array}{l}\text { Risk Ratio (M-H, Random, 95\% } \\
\text { Cl) }\end{array}$ & $0.63[0.45,0.88]$ \\
\hline 3.3 Major bleeding (6 months) & 5 & 2994 & $\begin{array}{l}\text { Risk Ratio (M-H, Random, 95\% } \\
\text { Cl) }\end{array}$ & $1.20[0.83,1.73]$ \\
\hline 3.3.1 GI tract cancer & 4 & 854 & $\begin{array}{l}\text { Risk Ratio (M-H, Random, 95\% } \\
\text { Cl) }\end{array}$ & $1.47[0.76,2.84]$ \\
\hline 3.3.2 Non-GI tract cancer & 4 & $\begin{array}{l}\text { Risk Ratio (M-H, Random, 95\% } \\
\text { Cl) }\end{array}$ & $1.05[0.63,1.73]$ \\
\hline 3.3.3 GI tract cancer not specified & 1 & 1853 & $\begin{array}{l}\text { Risk Ratio (M-H, Random, 95\% } \\
\text { Cl) }\end{array}$ & $0.20[0.01,4.04]$
\end{tabular}




\begin{tabular}{|c|c|c|c|c|}
\hline Outcome or subgroup title & No. of studies & $\begin{array}{l}\text { No. of partici- } \\
\text { pants }\end{array}$ & Statistical method & Effect size \\
\hline 3.4 Major GI bleeding ( 6 months) & 4 & 1838 & $\begin{array}{l}\text { Risk Ratio (M-H, Random, 95\% } \\
\mathrm{Cl} \text { ) }\end{array}$ & $1.16[0.62,2.17]$ \\
\hline $\begin{array}{l}\text { 3.5 Major upper GI bleeding ( } 6 \\
\text { months) }\end{array}$ & 4 & 1838 & $\begin{array}{l}\text { Risk Ratio (M-H, Random, 95\% } \\
\mathrm{Cl})\end{array}$ & $1.18[0.51,2.76]$ \\
\hline $\begin{array}{l}\text { 3.6 Major lower GI bleeding ( } 6 \\
\text { months) }\end{array}$ & 4 & 1838 & $\begin{array}{l}\text { Risk Ratio (M-H, Random, 95\% } \\
\mathrm{Cl})\end{array}$ & $1.10[0.43,2.80]$ \\
\hline $\begin{array}{l}\text { 3.7 Major non-GI bleeding ( } 6 \\
\text { months) }\end{array}$ & 4 & 1838 & $\begin{array}{l}\text { Risk Ratio (M-H, Random, 95\% } \\
\mathrm{Cl} \text { ) }\end{array}$ & $0.84[0.42,1.68]$ \\
\hline 3.8 Minor bleeding (6 months) & 5 & 2854 & $\begin{array}{l}\text { Risk Ratio (M-H, Random, 95\% } \\
\mathrm{Cl})\end{array}$ & $1.58[1.15,2.16]$ \\
\hline 3.9 Minor GI bleeding ( 6 months) & 2 & 1495 & $\begin{array}{l}\text { Risk Ratio (M-H, Random, 95\% } \\
\mathrm{Cl} \text { ) }\end{array}$ & $1.37[0.41,4.64]$ \\
\hline $\begin{array}{l}3.10 \text { Minor upper GI bleeding ( } 6 \\
\text { months) }\end{array}$ & 2 & 1495 & $\begin{array}{l}\text { Risk Ratio (M-H, Random, 95\% } \\
\mathrm{Cl} \text { ) }\end{array}$ & $1.03[0.04,25.97]$ \\
\hline $\begin{array}{l}\text { 3.11 Minor lower GI bleeding ( } 6 \\
\text { months) }\end{array}$ & 2 & 1495 & $\begin{array}{l}\text { Risk Ratio (M-H, Random, 95\% } \\
\mathrm{Cl} \text { ) }\end{array}$ & $1.54[0.72,3.27]$ \\
\hline $\begin{array}{l}3.12 \text { Minor non-GI bleeding ( } 6 \\
\text { months) }\end{array}$ & 2 & 1495 & $\begin{array}{l}\text { Risk Ratio (M-H, Random, 95\% } \\
\mathrm{Cl} \text { ) }\end{array}$ & $2.37[1.44,3.89]$ \\
\hline
\end{tabular}

Analysis 3.1. Comparison 3: Direct oral anticoagulants (DOAC) versus low molecular weight heparins (LMWH), Outcome 1: All-cause mortality (6 months)

\begin{tabular}{|c|c|c|c|c|c|c|c|c|}
\hline \multirow[b]{2}{*}{ Study or Subgroup } & \multicolumn{2}{|c|}{ DOAC } & \multicolumn{2}{|c|}{ LMWH } & \multirow[b]{2}{*}{ Weight } & Risk Ratio & \multirow{2}{*}{\multicolumn{2}{|c|}{$\begin{array}{c}\text { Risk Ratio } \\
\text { M-H, Random, 95\% CI }\end{array}$}} \\
\hline & Events & Total & Events & Total & & M-H, Random, 95\% CI & & \\
\hline Agnelli 2020 (Caravaggio) & 135 & 561 & 153 & 568 & $38.1 \%$ & $0.89[0.73,1.09]$ & & \\
\hline El Mokadem 2020 & 0 & 50 & 0 & 50 & & Not estimable & & \\
\hline McBane 2019 (ADAM-VTE) & 23 & 130 & 15 & 113 & $6.4 \%$ & $1.33[0.73,2.43]$ & & \\
\hline Raskob 2018 (HOKUSAI) & 140 & 509 & 127 & 507 & $36.6 \%$ & $1.10[0.89,1.35]$ & 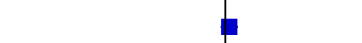 & \\
\hline Young 2018 (SELECT-D) & 48 & 186 & 56 & 180 & $18.8 \%$ & $0.83[0.60,1.15]$ & & \\
\hline Total (95\% CI) & & 1436 & & 1418 & $100.0 \%$ & $0.97[0.83,1.14]$ & 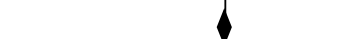 & \\
\hline Total events: & 346 & & 351 & & & & & \\
\hline \multicolumn{7}{|c|}{ Heterogeneity: $\mathrm{Tau}^{2}=0.01 ; \mathrm{Chi}^{2}=4.00, \mathrm{df}=3(\mathrm{P}=0.26) ; \mathrm{I}^{2}=25 \%$} & 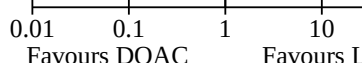 & $\begin{array}{r}100 \\
\text { MWH }\end{array}$ \\
\hline
\end{tabular}


Analysis 3.2. Comparison 3: Direct oral anticoagulants (DOAC) versus low molecular weight heparins (LMWH), Outcome 2: Recurrent VTE (6 months)

\begin{tabular}{|c|c|c|c|c|c|c|c|c|}
\hline \multirow[b]{2}{*}{ Study or Subgroup } & \multicolumn{2}{|c|}{ DOAC } & \multicolumn{2}{|c|}{ LMWH } & \multirow[b]{2}{*}{ Weight } & \multirow{2}{*}{$\begin{array}{c}\text { Risk Ratio } \\
\text { M-H, Random, 95\% CI }\end{array}$} & \multirow{2}{*}{\multicolumn{2}{|c|}{$\begin{array}{c}\text { Risk Ratio } \\
\text { M-H, Random, 95\% CI }\end{array}$}} \\
\hline & Events & Total & Events & Total & & & & \\
\hline Agnelli 2020 (Caravaggio) & 32 & 561 & 46 & 568 & $38.0 \%$ & $0.70[0.46,1.09]$ & $\rightarrow$ & \\
\hline El Mokadem 2020 & 3 & 50 & 5 & 50 & $5.6 \%$ & $0.60[0.15,2.38]$ & 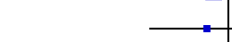 & \\
\hline McBane 2019 (ADAM-VTE) & 1 & 130 & 9 & 113 & $2.6 \%$ & $0.10[0.01,0.75]$ & & \\
\hline Raskob 2018 (HOKUSAI) & 34 & 509 & 46 & 507 & $39.1 \%$ & $0.74[0.48,1.13]$ & $\rightarrow$ & \\
\hline Young 2018 (SELECT-D) & 8 & 186 & 18 & 180 & $14.8 \%$ & $0.43[0.19,0.96]$ & & \\
\hline Total $(95 \%$ CI) & & 1436 & & 1418 & $100.0 \%$ & $0.63[0.45,0.88]$ & & \\
\hline Total events: & 78 & & 124 & & & & & \\
\hline \multicolumn{7}{|c|}{ Heterogeneity: $\mathrm{Tau}^{2}=0.03 ; \mathrm{Chi}^{2}=4.87, \mathrm{df}=4(\mathrm{P}=0.30) ; \mathrm{I}^{2}=18 \%$} & $\begin{array}{ll}0.01 & 0.1 \\
\end{array}$ & 10 \\
\hline Test for overall effect: $\mathrm{Z}=2.74$ & $(P=0.006)$ & & & & & & Favours DOAC & Favours I \\
\hline
\end{tabular}

Analysis 3.3. Comparison 3: Direct oral anticoagulants (DOAC) versus low molecular weight heparins (LMWH), Outcome 3: Major bleeding (6 months)

Test for subgroup differences: Not applicable

$\begin{array}{llll}\text { DOAC } & \text { LMWH } & \text { Risk Ratio } & \text { Risk Ratio }\end{array}$

Study or Subgroup

Events Total

Weight

M-H, Random, 95\% CI

$0.99[0.40,2.45]$

$0.55[0.10,2.96]$

3.18 [1.08, 9.37]

$1.80[0.64,5.09]$

$1.47[0.76,2.84]$

Young 2018 (SELECT-D)

Subtotal (95\% CI)

Total events:

$\begin{array}{rr}9 & 188 \\ 2 & 23 \\ 15 & 165 \\ 9 & 66 \\ & 442\end{array}$

$19 \quad 4.8 \%$

$140 \quad 11.6 \%$

$66 \quad 12.5 \%$

$412 \quad 45.6 \%$

$35 \quad 21$

Heterogeneity: $\mathrm{Tau}^{2}=0.13 ; \mathrm{Chi}^{2}=4.17, \mathrm{df}=3(\mathrm{P}=0.24) ; \mathrm{I}^{2}=28 \%$

Test for overall effect: $\mathrm{Z}=1.15(\mathrm{P}=0.25)$

\subsubsection{Non-GI tract cancer}

Agnelli 2020 (Caravaggio)

El Mokadem 2020

Raskob 2018 (HOKUSAI)

Young 2018 (SELECT-D)

Subtotal (95\% CI)

Total events:

$13 \quad 388$

$0 \quad 27$

$14 \quad 357$

$2 \quad 137$

909

29

Teterogeneity: $\mathrm{Tau}^{2}=0.00 ; \mathrm{Chi}^{2}=0.83, \mathrm{df}=$

Test for overall effect: $\mathrm{Z}=0.17(\mathrm{P}=0.86)$

\subsubsection{GI tract cancer not specified}

\section{McBane 2019 (ADAM-VTE)}

Subtotal (95\% CI)

Total events:

$\begin{array}{ll}0 & 145 \\ & 145\end{array}$
2
$142 \quad 1.5 \%$
$142 \quad 1.5 \%$

Heterogeneity: Not applicable

Test for overall effect: $\mathrm{Z}=1.06(\mathrm{P}=0.29)$

Total (95\% CI)

1496

$1498 \quad 100.0 \%$

Heterogeneity: $\mathrm{Tau}^{2}=0.00 ; \mathrm{Chi}^{2}=7.20, \mathrm{df}=8(\mathrm{P}=0.51) ; \mathrm{I}^{2}=0 \%$

Test for overall effect: $\mathrm{Z}=0.95(\mathrm{P}=0.34)$

Test for subgroup differences: $\mathrm{Chi}^{2}=1.98, \mathrm{df}=2(\mathrm{P}=0.37), \mathrm{I}^{2}=0 \%$
$0.94[0.45,1.97]$ $0.38[0.02,8.98]$

$1.16[0.55,2.43]$

$2.00[0.18,21.80]$

$1.05[0.63,1.73]$
M-H, Random, 95\% CI 
Analysis 3.4. Comparison 3: Direct oral anticoagulants (DOAC) versus low molecular weight heparins (LMWH), Outcome 4: Major GI bleeding (6 months)

\begin{tabular}{|c|c|c|c|c|c|c|c|}
\hline \multirow[b]{2}{*}{ Study or Subgroup } & \multicolumn{2}{|c|}{ DOAC } & \multicolumn{2}{|c|}{ LMWH } & \multirow[b]{2}{*}{ Weight } & \multirow{2}{*}{$\begin{array}{c}\text { Risk Ratio } \\
\text { M-H, Random, 95\% CI }\end{array}$} & \multirow{2}{*}{$\begin{array}{c}\text { Risk Ratio } \\
\text { M-H, Random, 95\% CI }\end{array}$} \\
\hline & Events & Total & Events & Total & & & \\
\hline Agnelli 2020 (Caravaggio) & 11 & 561 & 10 & 568 & $54.9 \%$ & $1.11[0.48,2.60]$ & \\
\hline El Mokadem 2020 & 2 & 50 & 3 & 50 & $13.0 \%$ & $0.67[0.12,3.82]$ & - \\
\hline McBane 2019 (ADAM-VTE) & 0 & 130 & 1 & 113 & $3.9 \%$ & $0.29[0.01,7.05]$ & \\
\hline Young 2018 (SELECT-D) & 8 & 186 & 4 & 180 & $28.3 \%$ & $1.94[0.59,6.32]$ & \\
\hline Total $(95 \%$ CI) & & 927 & & 911 & $100.0 \%$ & $1.16[0.62,2.17]$ & \\
\hline Total events: & 21 & & 18 & & & & \\
\hline \multirow{2}{*}{\multicolumn{7}{|c|}{$\begin{array}{l}\text { Heterogeneity: } \mathrm{Tau}^{2}=0.00 ; \mathrm{Chi}^{2}=1.84, \mathrm{df}=3(\mathrm{P}=0.61) ; \mathrm{I}^{2}=0 \% \\
\text { Test for overall effect: } \mathrm{Z}=0.45(\mathrm{P}=0.65)\end{array}$}} & $0.01 \quad 0.1$ \\
\hline & & & & & & & Favours DOAC \\
\hline
\end{tabular}

Analysis 3.5. Comparison 3: Direct oral anticoagulants (DOAC) versus low molecular weight heparins (LMWH), Outcome 5: Major upper GI bleeding (6 months)

\begin{tabular}{|c|c|c|c|c|c|c|c|}
\hline & & & & & & Risk Ratio & Risk Ratio \\
\hline Study or Subgroup & Events & Total & Events & Total & Weight & M-H, Random, 95\% CI & M-H, Random, 95\% CI \\
\hline
\end{tabular}

\begin{tabular}{lrrrrrr} 
Agnelli 2020 (Caravaggio) & 5 & 561 & 6 & 568 & $51.3 \%$ & $0.84[0.26,2.75]$ \\
El Mokadem 2020 & 0 & 50 & 0 & 50 & & Not estimable \\
McBane 2019 (ADAM-VTE) & 0 & 130 & 0 & 113 & & Not estimable \\
Young 2018 (SELECT-D) & 7 & 186 & 4 & 180 & $48.7 \%$ & $1.69[0.50,5.69]$ \\
Total (95\% CI) & & & & & & $\mathbf{9 1 1}$ \\
\hline
\end{tabular}

Total events: $\quad 12 \quad 10$

Heterogeneity: $\mathrm{Tau}^{2}=0.00 ; \mathrm{Chi}^{2}=0.65, \mathrm{df}=1(\mathrm{P}=0.42) ; \mathrm{I}^{2}=0 \%$

Test for overall effect: $\mathrm{Z}=0.39(\mathrm{P}=0.69)$

Test for subgroup differences: Not applicable

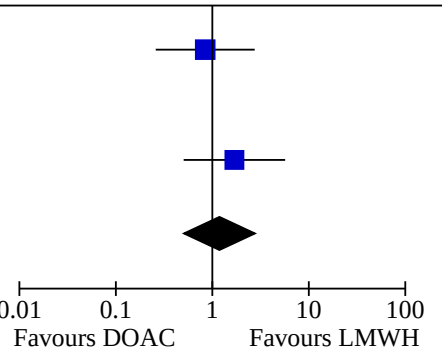

Analysis 3.6. Comparison 3: Direct oral anticoagulants (DOAC) versus low molecular weight heparins (LMWH), Outcome 6: Major lower GI bleeding (6 months)

\begin{tabular}{|c|c|c|c|c|c|c|c|}
\hline & \multicolumn{2}{|c|}{ DOAC } & \multicolumn{2}{|c|}{ LMWH } & \multicolumn{2}{|r|}{ Risk Ratio } & Risk Ratio \\
\hline Study or Subgroup & Events & Total & Events & Total & Weight & M-H, Random, 95\% CI & M-H, Random, 95\% CI \\
\hline
\end{tabular}

$\begin{array}{lrrrrrr}\text { Agnelli 2020 (Caravaggio) } & 6 & 561 & 4 & 568 & 54.6 \% & 1.52[0.43,5.35] \\ \text { El Mokadem 2020 } & 2 & 50 & 3 & 50 & 28.4 \% & 0.67[0.12,3.82] \\ \text { McBane 2019 (ADAM-VTE) } & 0 & 130 & 1 & 113 & 8.5 \% & 0.29[0.01,7.05] \\ \text { Young 2018 (SELECT-D) } & 1 & 186 & 0 & 180 & 8.5 \% & 2.90[0.12,70.82] \\ & & & & & & \mathbf{1 0 . 1 0}[\mathbf{0 . 4 3 , 2 . 8 0 ]} \\ \text { Total (95\% CI) } & & \mathbf{9 2 7} & & \mathbf{9 1 1} & \mathbf{1 0 0 . 0 \%} & \\ \text { Total events: } & 9 & & 8 & & \end{array}$

Heterogeneity: $\mathrm{Tau}^{2}=0.00 ; \mathrm{Chi}^{2}=1.59, \mathrm{df}=3(\mathrm{P}=0.66) ; \mathrm{I}^{2}=0 \%$

Test for overall effect: $\mathrm{Z}=0.21(\mathrm{P}=0.84)$

Test for subgroup differences: Not applicable

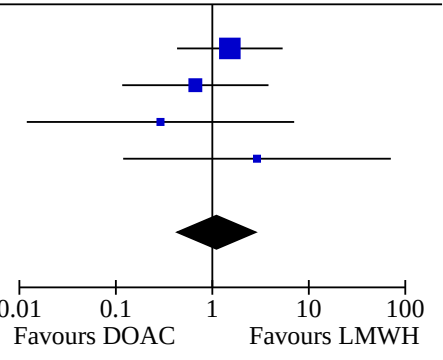


Analysis 3.7. Comparison 3: Direct oral anticoagulants (DOAC) versus low molecular weight heparins (LMWH), Outcome 7: Major non-GI bleeding (6 months)

\begin{tabular}{|c|c|c|c|c|c|c|c|}
\hline \multirow[b]{2}{*}{ Study or Subgroup } & \multicolumn{2}{|c|}{ DOAC } & \multicolumn{2}{|c|}{ LMWH } & \multirow[b]{2}{*}{ Weight } & \multirow{2}{*}{$\begin{array}{c}\text { Risk Ratio } \\
\text { M-H, Random, 95\% CI }\end{array}$} & \multirow{2}{*}{$\begin{array}{c}\text { Risk Ratio } \\
\text { M-H, Random, 95\% CI }\end{array}$} \\
\hline & Events & Total & Events & Total & & & \\
\hline Agnelli 2020 (Caravaggio) & 11 & 561 & 13 & 568 & $75.5 \%$ & $0.86[0.39,1.90]$ & \\
\hline El Mokadem 2020 & 0 & 50 & 1 & 50 & $4.7 \%$ & $0.33[0.01,7.99]$ & \\
\hline McBane 2019 (ADAM-VTE) & 0 & 130 & 1 & 113 & $4.7 \%$ & $0.29[0.01,7.05]$ & \\
\hline Young 2018 (SELECT-D) & 3 & 186 & 2 & 180 & $15.1 \%$ & $1.45[0.25,8.59]$ & \\
\hline Total $(95 \%$ CI) & & 927 & & 911 & $100.0 \%$ & $0.84[0.42,1.68]$ & \\
\hline Total events: & 14 & & 17 & & & & \\
\hline \multirow{2}{*}{\multicolumn{7}{|c|}{$\begin{array}{l}\text { Heterogeneity: } \mathrm{Tau}^{2}=0.00 ; \mathrm{Chi}^{2}=1.12, \mathrm{df}=3(\mathrm{P}=0.77) ; \mathrm{I}^{2}=0 \% \\
\text { Test for overall effect: } \mathrm{Z}=0.48(\mathrm{P}=0.63)\end{array}$}} & $0.01 \quad 0.1$ \\
\hline & & & & & & & Favours DOAC \\
\hline
\end{tabular}

Test for subgroup differences: Not applicable

Analysis 3.8. Comparison 3: Direct oral anticoagulants (DOAC) versus low molecular weight heparins (LMWH), Outcome 8: Minor bleeding (6 months)

\begin{tabular}{|c|c|c|c|c|c|c|c|}
\hline & \multicolumn{2}{|c|}{ DOAC } & \multicolumn{2}{|c|}{ LMWH } & \multicolumn{2}{|r|}{ Risk Ratio } & Risk Ratio \\
\hline Study or Subgroup & Events & Total & Events & Total & Weight & M-H, Random, 95\% CI & M-H, Random, 95\% CI \\
\hline
\end{tabular}

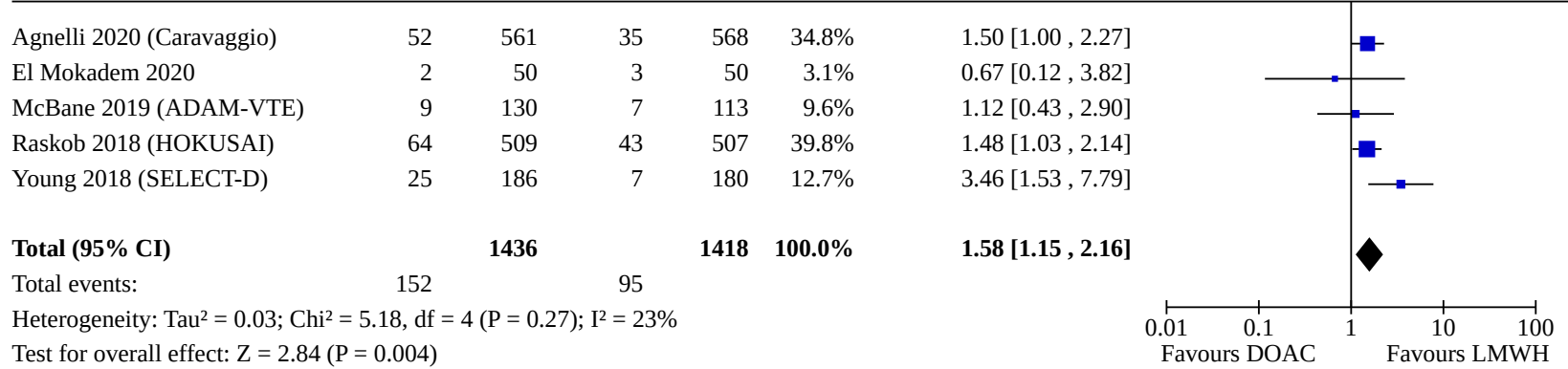

Test for subgroup differences: Not applicable

Analysis 3.9. Comparison 3: Direct oral anticoagulants (DOAC) versus low molecular weight heparins (LMWH), Outcome 9: Minor GI bleeding ( 6 months)

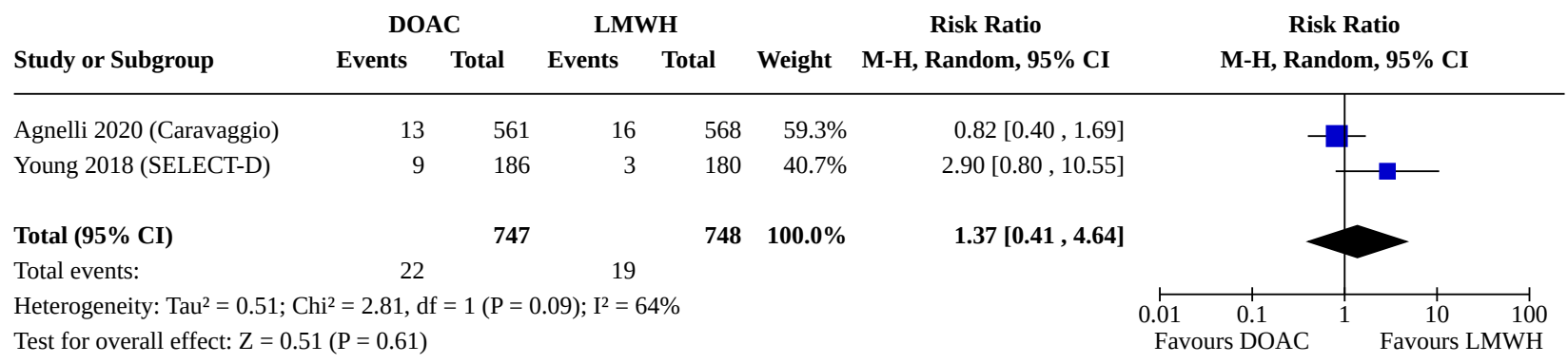


Analysis 3.10. Comparison 3: Direct oral anticoagulants (DOAC) versus low molecular weight heparins (LMWH), Outcome 10: Minor upper GI bleeding (6 months)

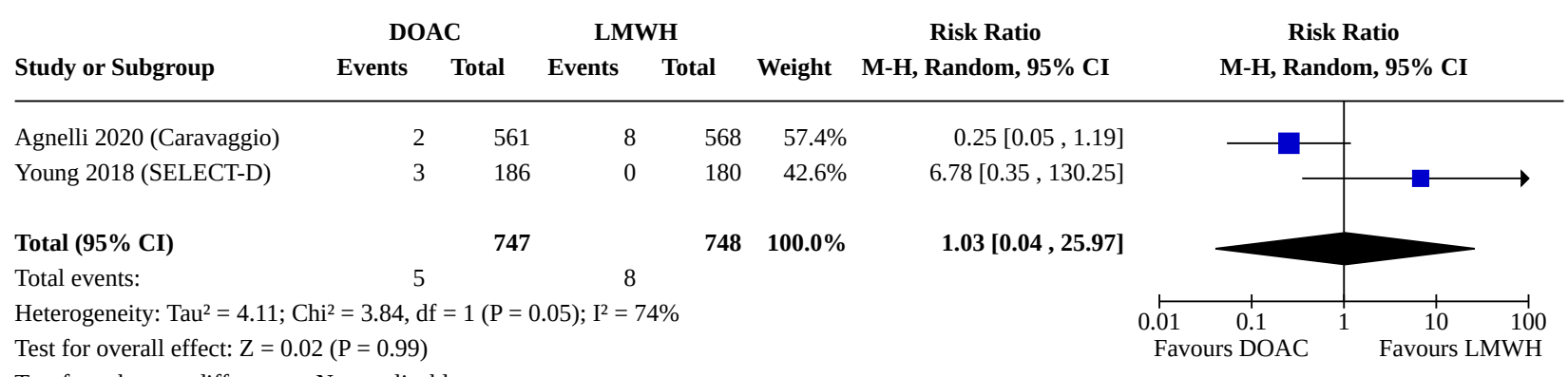

Test for subgroup differences: Not applicable

Analysis 3.11. Comparison 3: Direct oral anticoagulants (DOAC) versus low molecular weight heparins (LMWH), Outcome 11: Minor lower GI bleeding (6 months)

\begin{tabular}{|c|c|c|c|c|c|c|c|}
\hline & \multicolumn{2}{|c|}{ DOAC } & \multicolumn{2}{|c|}{ LMWH } & \multicolumn{2}{|r|}{ Risk Ratio } & Risk Ratio \\
\hline Study or Subgroup & Events & Total & Events & Total & Weight & M-H, Random, 95\% CI & M-H, Random, 95\% CI \\
\hline
\end{tabular}

\begin{tabular}{|c|c|c|c|c|c|c|}
\hline Agnelli 2020 (Caravaggio) & 11 & 561 & 8 & 568 & $69.7 \%$ & $1.39[0.56,3.44]$ \\
\hline Young 2018 (SELECT-D) & 6 & 186 & 3 & 180 & $30.3 \%$ & $1.94[0.49,7.62]$ \\
\hline Total (95\% CI) & & 747 & & 748 & $100.0 \%$ & $1.54[0.72,3.27]$ \\
\hline Total events: & 17 & & 11 & & & \\
\hline
\end{tabular}

Heterogeneity: Tau ${ }^{2}=0.00 ; \mathrm{Chi}^{2}=0.15, \mathrm{df}=1(\mathrm{P}=0.69) ; \mathrm{I}^{2}=0 \%$

Test for overall effect: $\mathrm{Z}=1.12(\mathrm{P}=0.26)$

Test for subgroup differences: Not applicable

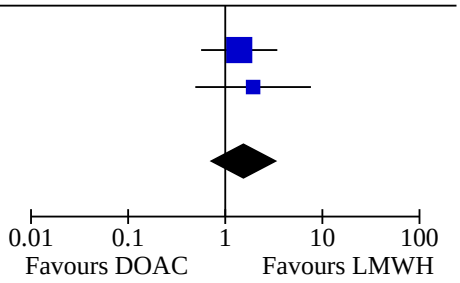

Analysis 3.12. Comparison 3: Direct oral anticoagulants (DOAC) versus low molecular weight heparins (LMWH), Outcome 12: Minor non-GI bleeding (6 months)

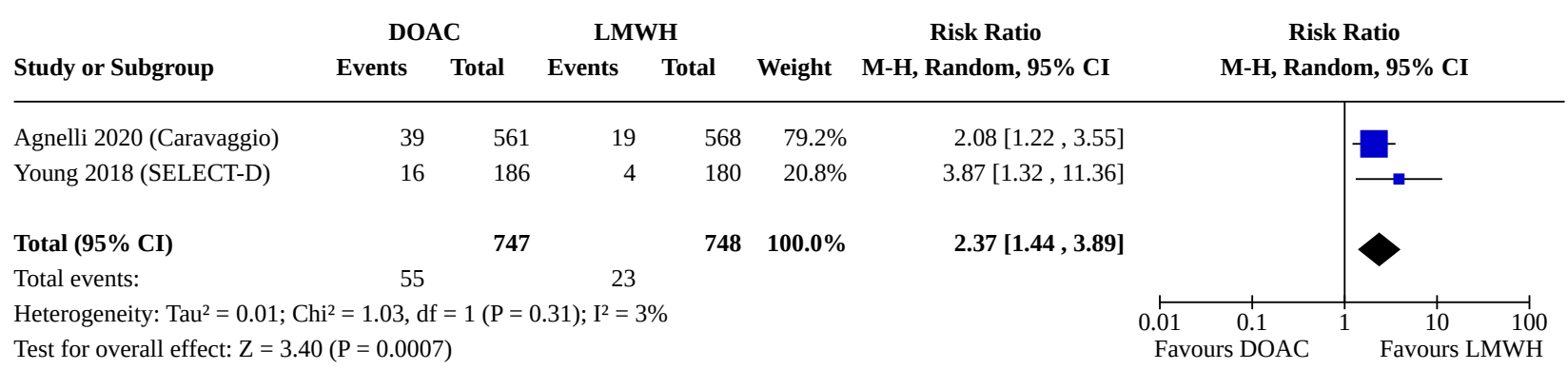

Test for subgroup differences: Not applicable

Comparison 4. Idraparinux versus vitamin $\mathrm{K}$ antagonists (VKA)

\begin{tabular}{lllll}
\hline Outcome or subgroup title & No. of studies & $\begin{array}{l}\text { No. of partici- } \\
\text { pants }\end{array}$ & Statistical method & Effect size \\
\hline $\begin{array}{l}\text { 4.1 All-cause mortality (up to 6 } \\
\text { months) }\end{array}$ & 1 & 284 & $\begin{array}{l}\text { Risk Ratio (M-H, Random, 95\% } \\
\text { Cl) }\end{array}$ & $1.11[0.78,1.59]$ \\
\hline 4.2 Recurrent VTE (up to 6 months) & 1 & 270 & $\begin{array}{l}\text { Risk Ratio (M-H, Random, 95\% } \\
\text { Cl) }\end{array}$ & $0.46[0.16,1.32]$ \\
\hline \hline
\end{tabular}




\begin{tabular}{llllll}
\hline Outcome or subgroup title & No. of studies & $\begin{array}{l}\text { No. of partici- } \\
\text { pants }\end{array}$ & Statistical method & Effect size \\
\hline 4.3 Major bleeding (up to 6 months) & 1 & 270 & $\begin{array}{l}\text { Risk Ratio (M-H, Random, 95\% } \\
\text { Cl) }\end{array}$ & $1.11[0.35,3.56]$ \\
\hline 4.4 Minor bleeding (up to 6 months) & 1 & 270 & $\begin{array}{l}\text { Risk Ratio (M-H, Random, 95\% } \\
\text { Cl) }\end{array}$ & $0.70[0.30,1.60]$ \\
\hline
\end{tabular}

Analysis 4.1. Comparison 4: Idraparinux versus vitamin $\mathrm{K}$ antagonists (VKA), Outcome 1: All-cause mortality (up to 6 months)

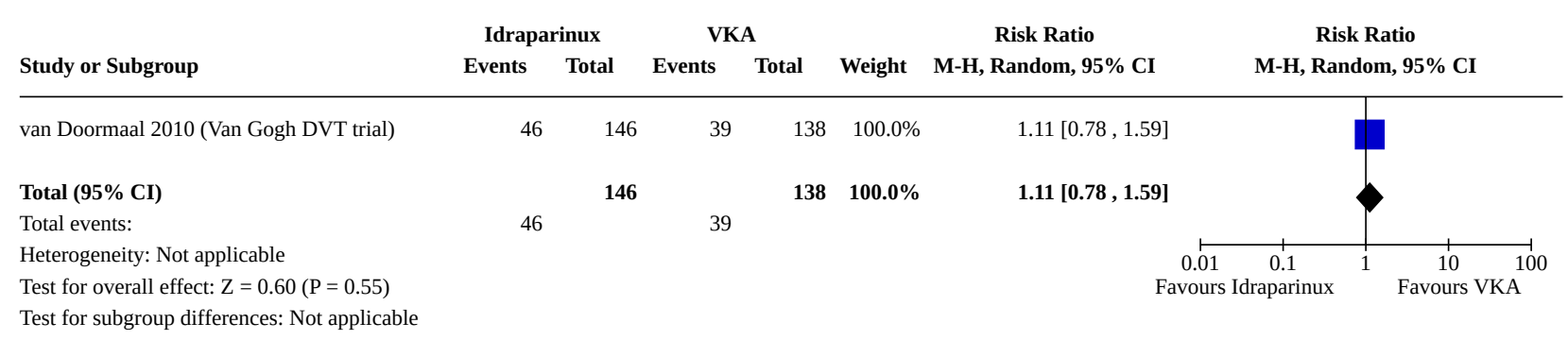

Analysis 4.2. Comparison 4: Idraparinux versus vitamin $K$ antagonists (VKA), Outcome 2: Recurrent VTE (up to 6 months)

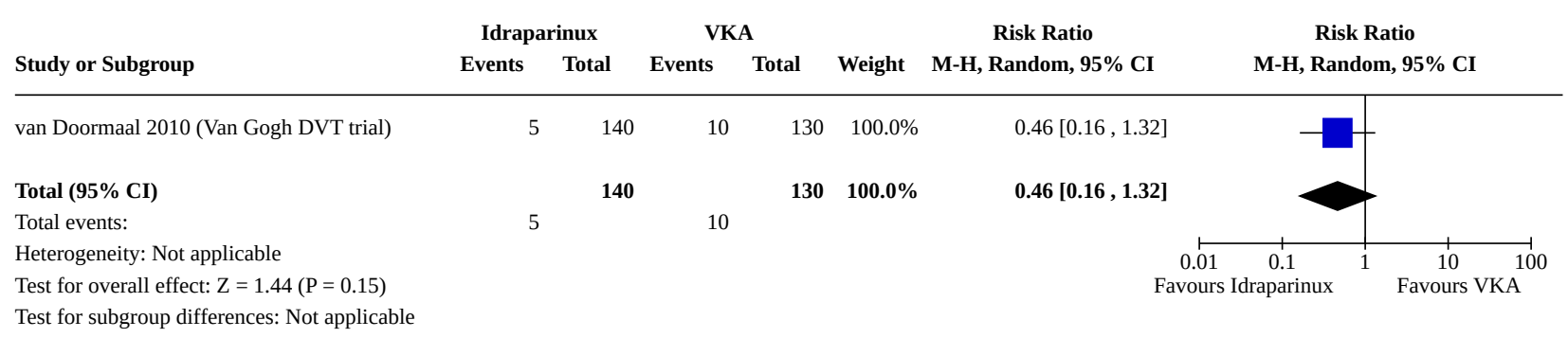

Analysis 4.3. Comparison 4: Idraparinux versus vitamin $\mathrm{K}$ antagonists (VKA), Outcome 3: Major bleeding (up to 6 months)

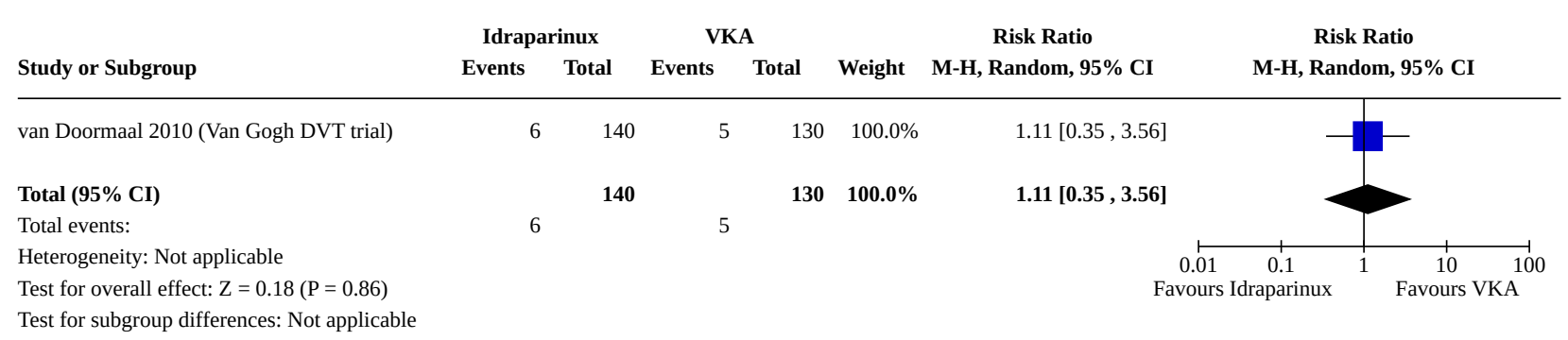


Analysis 4.4. Comparison 4: Idraparinux versus vitamin $K$ antagonists (VKA), Outcome 4: Minor bleeding (up to 6 months)

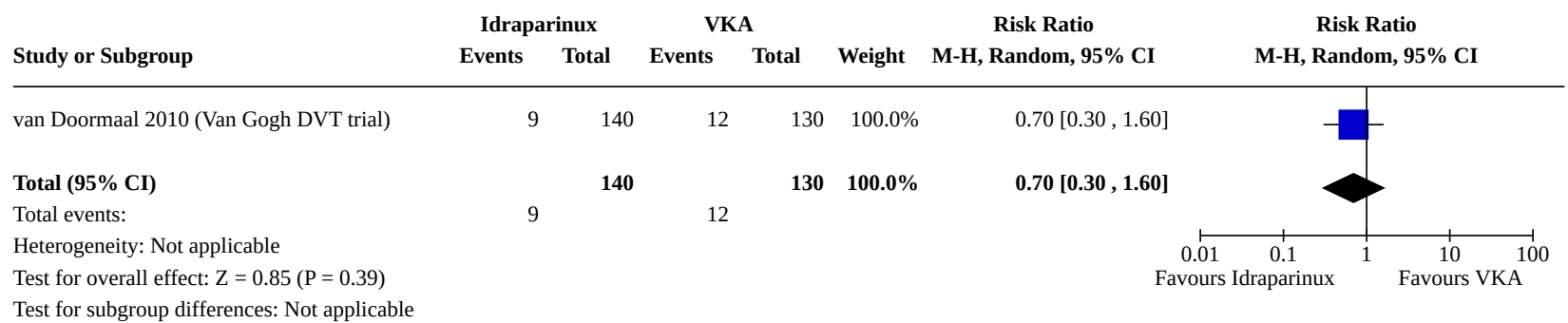

\section{ADDITIONAL TABLES}

Table 1. Glossary

\begin{tabular}{|c|c|}
\hline Term & Definition \\
\hline Adjuvant therapy & $\begin{array}{l}\text { A therapy given in addition to the primary treatment to decrease the risk of the cancer recurrence } \\
\text { or to assist in the cure. }\end{array}$ \\
\hline Anticoagulation & The process of hindering the clotting of blood especially by treatment with an anticoagulant. \\
\hline Antithrombotic & Used against or tending to prevent thrombosis (clotting) \\
\hline Coagulation & Clotting \\
\hline $\begin{array}{l}\text { Direct oral anticoagulants } \\
\text { (DOAC) }\end{array}$ & $\begin{array}{l}\text { Also known as NOACs are anticoagulant medications that require less monitoring compared to the } \\
\text { traditional anticoagulants. }\end{array}$ \\
\hline Deep vein thrombosis (DVT) & $\begin{array}{l}\text { A condition marked by the formation of a thrombus within a deep vein (as of the leg or pelvis) that } \\
\text { may be asymptomatic or be accompanied by symptoms (as swelling and pain) and that is poten- } \\
\text { tially life-threatening if dislodgment of the thrombus results in pulmonary embolism. }\end{array}$ \\
\hline Fondaparinux & An anticoagulant medication \\
\hline Hemostatic system & The system that shortens the clotting time of blood and stops bleeding. \\
\hline Heparin & $\begin{array}{l}\text { An enzyme occurring especially in the liver and lungs that prolongs the clotting time of blood by } \\
\text { preventing the formation of fibrin. } 2 \text { forms of heparin that are used as anticoagulant medications } \\
\text { are: unfractionated heparin (UFH) and low molecular weight heparins (LMWH). }\end{array}$ \\
\hline Impedance plethysmography & $\begin{array}{l}\text { A technique that measures the change in blood volume (venous blood volume as well as the pulsa- } \\
\text { tion of the arteries) for a specific body segment }\end{array}$ \\
\hline Kappa statistic & $\begin{array}{l}\text { A measure of degree of nonrandom agreement between observers, measurements of a specific cat- } \\
\text { egorical variable, or both. }\end{array}$ \\
\hline Metastasis & The spread of a cancer cells from the initial or primary site of disease to another part of the body. \\
\hline Parenteral nutrition & The practice of feeding a person intravenously, circumventing the gastrointestinal tract. \\
\hline Pulmonary embolism (PE) & $\begin{array}{l}\text { Embolism of a pulmonary artery or one of its branches that is produced by foreign matter and most } \\
\text { often a blood clot originating in a vein of the leg or pelvis and that is marked by labored breathing, } \\
\text { chest pain, fainting, rapid heart rate, cyanosis, shock and sometimes death. }\end{array}$ \\
\hline
\end{tabular}


Table 1. Glossary (Continued)

Thrombocytopenia

Thrombosis
Persistent decrease in the number of blood platelets that is often associated with hemorrhagic conditions.

Vitamin $\mathrm{K}$ antagonists $\quad$ Anticoagulant medications. Warfarin is a vitamin $\mathrm{K}$ antagonist.

\section{AP PE N D I C E S}

\section{Appendix 1. Living systematic review protocol}

The methods outlined below are specific to maintaining the review as a living systematic review in the Cochrane Library (Synnot 2017). They will be implemented immediately upon publication of this update. Core review methods, such as the criteria for considering studies in the review and assessment of risk of bias, are unchanged. As such, below we outline only those areas of the methods for which additional or different activities are planned or rules apply.

\section{Search methods for identification of studies}

We will rerun the majority of searches monthly. For electronic databases and other electronic sources (CENTRAL, MEDLINE, Embase), we have set up auto-alerts to deliver a monthly search yield by email. We will search the remaining resources (conference proceedings of the American Society of Clinical Oncology (ASCO); the American Society of Haematology (ASH); and clinicaltrials.gov) on a bi-yearly basis. For that purpose, we will note when these conference proceedings are published.

As additional steps to inform the living systematic review, we will contact corresponding authors of ongoing studies as they are identified and ask them to advise when results are available, and to share early or unpublished data. We will contact the corresponding authors of any newly included studies for advice as to other relevant studies.We will manually screen the reference list of any newly included studies, and identified relevant guidelines and systematic reviews. .

We will review search methods and strategies approximately yearly, to ensure they reflect any terminology changes in the topic area, or in the databases.

\section{Selection of studies}

We will immediately screen any new citations retrieved by the monthly searches. As the first step of monthly screening, we will apply the machine learning classifier (RCT model) available in the Cochrane Register of Studies (CSR-web; Wallace 2017). The classifier assigns a probability (from 0 to 100) to each citation for being a true RCT. For citations that are assigned a probability score of less than 10 , the machine learning classifier currently has a specificity/recall of $99.987 \%$ (Thomas 2017). For citations assigned a score from 10 to 100 , we will screen them in duplicate and independently. Citations that score 9 or less will be screened by Cochrane Crowd. Any citations that are deemed to be potential RCTs by Cochrane Crowd will be returned to the authors for screening.

\section{Data synthesis}

Whenever new evidence (studies, data or information) that meets the review inclusion criteria is identified, we will immediately assess risk of bias and extract the data and incorporate it in the synthesis, as appropriate. We will not adjust the meta-analyses to account for multiple testing given the methods related to frequent updating of meta-analyses are under development (Simmonds 2017).

\section{Other}

We will review the review scope and methods approximately yearly, or more frequently if appropriate, in light of potential changes in the topic area, or the evidence being included in the review (e.g. additional comparisons, interventions or outcomes, or new review methods available).

\section{Appendix 2. Full search strategies for the electronic databases: update December 2020}

Database Strategy


(Continued)

CENTRAL (the Cochrane Library, latest issue)

\section{RCTsearch strategy:}

1. exp Anticoagulants/

(anticoagulant ${ }^{\star}$ or anti-coagulant*).tw.

(Heparin or Adomiparin or alpha-Heparin or Arteven or "AVE-5026" or CY 222 or "Depo-Heparin" or "EINECS 232-681-7" or Fluxum or "Hed-heparin" or Hepathrom or HSDB 3094 or KB 101 or "Lipo-hepin" or M 118 or "M 118REH" or M118 or Octaparin or OP 386 or OP 622 or Pabyrin or Pularin or Subeparin or Sublingula or Thromboliquine or Triofiban or "UNII-1K5KDI46KZ" or "UNII-4QW4AN84NQ" or "UNII-5R0L1D739E" or "UNII-7UQ7X4Y489" or "UNII-9816XA9004" or "UNIIE47CONF7LV" or "UNII-M316WT19D8" or "UNII-P776JQ4R2F" or "UNII-S79008V79F" or "UNIIT2410KM04A" or "UNII-V72OT3K19I" or "UNII-VLOL558GCB" or Vetren or Vitrum AB or enoxaparin* or klexane or lovenox or fragmin* or normiflo or logiparin or innohep or danaproid or danaparoid or orgaran or antixarin or hibor or zibor or ivor or badyket or lohepa or lowhepa or seleparin* or tedelgliparin or lomoparan or orgaran or sulodexide or zivor or embolex or xaparin or fondaparinux or Arixtra or UFH or Hepalean or Calcilean or Calciparine or "Hep-lock" or enoxaparin* or klexane or lovenox or fragmin* or normiflo or logiparin or innohep or danaproid or danaparoid or orgaran or antixarin or hibor or zibor or ivor or badyket or lohepa or lowhepa or seleparin* or tedelgliparin or lomoparan or orgaran or sulodexide or zivor or embolex or xaparin or fondaparinux or Arixtra or UFH or Hepalean or Panheprin).mp.

2. $\left(\mathrm{LMWH}^{\star}\right.$ or heparin ${ }^{\star}$ or nadroparin ${ }^{\star}$ or frixiparin ${ }^{\star}$ or enoxaparin ${ }^{\star}$ or clexane or klexane or lovenox or dalteparin or fragmin or ardeparin* or normiflo or tinzaparin or logiparin or innohep or certoparin or sandoparin or reviparin or clivarin* or danaproid or danaparoid or orgaran or antixarin or bemiparin* or hibor or zibor or ivor or badyket or semuloparin or parnaparin or tedelparin or fluxum or lohepa or lowhepa or parvoparin or seleparin* or tedelgliparin or lomoparan or orgaran or sulodexide or zivor or embolex or xaparin or clivarine or fondaparinux or Arixtra or UFH or Hepalean or Calcilean or Calciparine or Liquaemin or Liquemin or Multiparin or Novoheparin or Eparina or Hep-lock or Heparinate or Heparinic acid or Panheprin or Hepalean or Heparin Leo or Heparin Lock).mp.

3. (FR-860 or FR 860 or FR860 or PK-10,169 or PK 10,169 or PK10,169 or PK-10169 or PK 10169 or PK10169 or EMT-967 or EMT 967 or EMT967 or EMT-966 or EMT 966 or EMT966 or CY 216 or CY-216 or CY216 or LMF CY-216 or LMF CY 216 or LMF CY216).mp.

\section{4. exp Coumarins/}

(coumarin ${ }^{\star}$ or chromonar or coumestrol or esculin or isocoumarin* or psoralens or pyranocoumarins or umbelliferones).tw.

5. (4-Hydroxycoumarin* or warfarin* or acenocoumarol or nicoumalone or sinthrome or Sintrom or phenindione or dicoumarol or coumadin or phenprocoumon or phepromaron or ethyl-biscoumacetate or phenindione or Diphenadione or Tioclomarol or Racumi or Marcoumar or Marcumar or Falithrom or Jantoven or vitamin $\mathrm{K}$ antagonist* or VKA or fluindione or difenacoum or coumatetralyl or coumadin* or warfant or marevan or aldocumar).mp.

6. (Dermatan Sulfate or (Chondroitin Sulfate adj B) or Dermatan Sulfphate or DS 435 or MF-701 or OP-370 or b-Heparin or Mistral or Venorix).mp.

7. (thrombin adj inhibitor $\left.{ }^{\star}\right) \cdot m p$.

8. (factor Xa inhibitor* or antithrombin* or anticoagul*).mp.

9. (rivaroxaban or Xarelto or apixaban or Eliquis or dabigatran etexilate or Edoxaban or Savaysa or Betrixaban or ximelagatran or pradaxa or lixiana or exanta or Darexaban or Otamixaban* or Razaxaban or Bivalirudin or Desirudin or Lepirudin or Melagatran or YM 150 or Iprivask or argatrovan or pradax* or Xarelto or BIBR-953 or BIBR-953ZW or BAY 59-7939 or BMS-562247 or DU-176 or DU-176b).mp.

\section{RIVAROXABAN/}


11. DABIGATRAN/ (BIBR 953 or BIBR 953 ZW or Dabigatran or HSDB 8062 or Pradaxa or UNIIIOVM4M70GC)

12. (target specific oral anticoagulant ${ }^{\star}$ or target-specific oral anticoagulant ${ }^{\star}$ or TSOAC ${ }^{\star}$ or new oral anticoagulant ${ }^{\star}$ or novel oral anticoagulant ${ }^{\star}$ or NOAC* or direct-acting oral anticoagulant ${ }^{\star}$ or direct acting oral anticoagulant ${ }^{\star}$ or direct oral anticoagulant ${ }^{\star}$ or DOAC*).ti,ab,kw.

\section{1 or 2 or 3 or 4 or 5 or 6 or 7 or 8 or 9 or 10 or 11 or 12}

\section{4. exp Neoplasms/}

15. (malignan* or neoplasm ${ }^{\star}$ or cancer ${ }^{\star}$ or carcinoma* or adenocarcinoma* or tumour* or tumor ${ }^{\star}$ or glioma* or myeloma* or lymphoma* or leukemia* or leukaemia* or epithelioma* or adenoma*).tw.

\section{14 or 15}

\section{13 and 16}

18. randomised controlled trial.pt.

19. controlled clinical trial.pt.

20. randomized.ab.

21. placebo.ab.

22. clinical trials as topic.sh.

23. randomly.ab.

24. trial.ti.

25.18 or 19 or 20 or 21 or 22 or 23 or 24

26. (animals not (humans and animals)).sh.

\subsection{5 not 26}

28. 17 and 27

\section{Systematic Review search strategy:}

1. exp Anticoagulants/

2. (anticoagulant ${ }^{\star}$ or anti-coagulant $\left.{ }^{\star}\right)$.tw.

3. (Heparin or Adomiparin or alpha-Heparin or Arteven or "AVE-5026" or CY 222 or "Depo-Heparin" or "EINECS 232-681-7" or Fluxum or "Hed-heparin" or Hepathrom or HSDB 3094 or KB 101 or "Lipo-hepin" or M 118 or "M 118REH" or M118 or Octaparin or OP 386 or OP 622 or Pabyrin or Pularin or Subeparin or Sublingula or Thromboliquine or Triofiban or "UNII-1K5KDI46KZ" or "UNII-4QW4AN84NQ" or "UNII-5R0L1D739E" or "UNII-7UQ7X4Y489" or "UNII-9816XA9004" or "UNIIE47C0NF7LV" or "UNII-M316WT19D8" or "UNII-P776JQ4R2F" or "UNII-S79008V79F" or "UNIIT2410KM04A" or "UNII-V72OT3K19I" or "UNII-VL0L558GCB" or Vetren or Vitrum AB or enoxaparin* or klexane or lovenox or fragmin* or normiflo or logiparin or innohep or danaproid or danaparoid or orgaran or antixarin or hibor or zibor or ivor or badyket or lohepa or lowhepa or seleparin* or tedelgliparin or lomoparan or orgaran or sulodexide or zivor or embolex or xaparin or fondaparinux or Arixtra or UFH or Hepalean or Calcilean or Calciparine or "Hep-lock" or enoxaparin* or klexane or lovenox or fragmin* or normiflo or logiparin or innohep or danaproid or danaparoid or orgaran or antixarin or hibor or zibor or ivor or badyket or lohepa or lowhepa or seleparin* or tedelgliparin or lomoparan or orgaran or sulodexide or zivor or embolex or xaparin or fondaparinux or Arixtra or UFH or Hepalean or Panheprin).mp.

4. $\left(\mathrm{LMWH}^{\star}\right.$ or heparin* or nadroparin* or frixiparin* or enoxaparin* or clexane or klexane or lovenox or dalteparin or fragmin or ardeparin* or normiflo or tinzaparin or logiparin or innohep or cer- 
toparin or sandoparin or reviparin or clivarin ${ }^{\star}$ or danaproid or danaparoid or orgaran or antixarin or bemiparin* or hibor or zibor or ivor or badyket or semuloparin or parnaparin or tedelparin or fluxum or lohepa or lowhepa or parvoparin or seleparin* or tedelgliparin or lomoparan or orgaran or sulodexide or zivor or embolex or xaparin or clivarine or fondaparinux or Arixtra or UFH or Hepalean or Calcilean or Calciparine or Liquaemin or Liquemin or Multiparin or Novoheparin or Eparina or Hep-lock or Heparinate or Heparinic acid or Panheprin or Hepalean or Heparin Leo or Heparin Lock).mp.

\section{5. (FR-860 or FR 860 or FR860 or PK-10,169 or PK 10,169 or PK10,169 or PK-10169 or PK 10169 or PK10169 or EMT-967 or EMT 967 or EMT967 or EMT-966 or EMT 966 or EMT966 or CY 216 or CY-216 or CY216 or LMF CY-216 or LMF CY 216 or LMF CY216).mp.}

\section{6. exp Coumarins/}

7. (coumarin* or chromonar or coumestrol or esculin or isocoumarin* or psoralens or pyranocoumarins or umbelliferones).tw.

8. (4-Hydroxycoumarin* or warfarin* or acenocoumarol or nicoumalone or sinthrome or Sintrom or phenindione or dicoumarol or coumadin or phenprocoumon or phepromaron or ethyl-biscoumacetate or phenindione or Diphenadione or Tioclomarol or Racumi or Marcoumar or Marcumar or Falithrom or Jantoven or vitamin $\mathrm{K}$ antagonist ${ }^{\star}$ or VKA or fluindione or difenacoum or coumatetralyl or coumadin* or warfant or marevan or aldocumar).mp.

9. (Dermatan Sulfate or (Chondroitin Sulfate adj B) or Dermatan Sulfphate or DS 435 or MF-701 or OP-370 or b-Heparin or Mistral or Venorix).mp.

10. (thrombin adj inhibitor $\left.{ }^{\star}\right) \cdot \mathrm{mp}$.

11. (factor Xa inhibitor ${ }^{\star}$ or antithrombin ${ }^{\star}$ or anticoagul $\left.{ }^{\star}\right) . \mathrm{mp}$.

12. (rivaroxaban or Xarelto or apixaban or Eliquis or dabigatran etexilate or Edoxaban or Savaysa or Betrixaban or ximelagatran or pradaxa or lixiana or exanta or Darexaban or Otamixaban* or Razaxaban or Bivalirudin or Desirudin or Lepirudin or Melagatran or YM 150 or Iprivask or argatrovan or pradax* or Xarelto or BIBR-953 or BIBR-953ZW or BAY 59-7939 or BMS-562247 or DU-176 or DU-176b).mp.

\section{RIVAROXABAN/}

\section{DABIGATRAN/}

15. (BIBR 953 or BIBR 953 ZW or Dabigatran or HSDB 8062 or Pradaxa or UNII-IOVM4M70GC).mp. [ $\mathrm{mp}=$ title, abstract, original title, name of substance word, subject heading word, floating subheading word, keyword heading word, organism supplementary concept word, protocol supplementary concept word, rare disease supplementary concept word, unique identifier, synonyms]

16. (target specific oral anticoagulant* or target-specific oral anticoagulant ${ }^{\star}$ or TSOAC ${ }^{\star}$ or new oral anticoagulant ${ }^{\star}$ or novel oral anticoagulant ${ }^{\star}$ or NOAC* or direct-acting oral anticoagulant ${ }^{\star}$ or direct acting oral anticoagulant ${ }^{\star}$ or direct oral anticoagulant ${ }^{\star}$ or DOAC $) . t i, a b, k w$.

\section{1 or 2 or 3 or 4 or 5 or 6 or 7 or 8 or 9 or 10 or 11 or 12 or 13 or 14 or 15 or 16}

18. exp Neoplasms/

19. (malignan ${ }^{\star}$ or neoplasm ${ }^{\star}$ or cancer ${ }^{\star}$ or carcinoma* or adenocarcinoma* or tumour ${ }^{\star}$ or tumor ${ }^{\star}$ or glioma* or myeloma* or lymphoma* or leukemia* or leukaemia* or epithelioma* or adeno$\left.m a^{\star}\right)$.tw.

\section{18 or 19}

21. 17 and 20

22. Meta-Analysis as Topic/

23. meta analy\$.tw. 


\section{4. metaanaly\$.tw.}

25. Meta-Analysis/

26. (systematic adj (review\$1 or overview\$1)).tw.

27. exp Review Literature as Topic/

28. 22 or 23 or 24 or 25 or 26 or 27

29. cochrane.ab.

30. embase.ab.

31. (psychlit or psyclit).ab.

32. (psychinfo or psycinfo).ab.

33. (cinahl or cinhal).ab.

34. science citation index.ab.

35. bids.ab.

36. cancerlit.ab.

37.29 or 30 or 31 or 32 or 33 or 34 or 35 or 36

38. reference list\$.ab.

39. bibliograph\$.ab.

40. hand-search\$.ab.

41. relevant journals.ab.

42. manual search\$.ab.

43.38 or 39 or 40 or 41 or 42

44. selection criteria.ab.

45. data extraction.ab.

46. 44 or 45

47. Review/

48. 46 and 47

49. Comment/

50. Letter/

51. Editorial/

52. animal/

53. human/

54. 52 not (52 and 53)

55.49 or 50 or 51 or 54

56. 28 or 37 or 43 or 48

57.56 not 55 


\section{21 and 57}

\section{RCT search strategy:}

1. exp anticoagulant agent/

2. (anticoagulant ${ }^{\star}$ or anti-coagulant $\left.{ }^{\star}\right)$.tw.

3. (Heparin or Adomiparin or alpha-Heparin or Arteven or "AVE-5026" or CY 222 or "Depo-Heparin" or "EINECS 232-681-7" or Fluxum or "Hed-heparin" or Hepathrom or HSDB 3094 or KB 101 or "Lipo-hepin" or M 118 or "M 118REH" or M118 or Octaparin or OP 386 or OP 622 or Pabyrin or Pularin or Subeparin or Sublingula or Thromboliquine or Triofiban or "UNII-1K5KDI46KZ" or "UNII-4QW4AN84NQ" or "UNII-5R0L1D739E" or "UNII-7UQ7X4Y489" or "UNII-9816XA9004" or "UNIIE47C0NF7LV" or "UNII-M316WT19D8" or "UNII-P776JQ4R2F" or "UNII-S79008V79F" or "UNIIT2410KM04A" or "UNII-V72OT3K19I" or "UNII-VL0L558GCB" or Vetren or Vitrum AB or enoxaparin* or klexane or lovenox or fragmin* or normiflo or logiparin or innohep or danaproid or danaparoid or orgaran or antixarin or hibor or zibor or ivor or badyket or lohepa or lowhepa or seleparin* or tedelgliparin or lomoparan or orgaran or sulodexide or zivor or embolex or xaparin or fondaparinux or Arixtra or UFH or Hepalean or Calcilean or Calciparine or "Hep-lock" or enoxaparin* or klexane or lovenox or fragmin* or normiflo or logiparin or innohep or danaproid or danaparoid or orgaran or antixarin or hibor or zibor or ivor or badyket or lohepa or lowhepa or seleparin* or tedelgliparin or lomoparan or orgaran or sulodexide or zivor or embolex or xaparin or fondaparinux or Arixtra or UFH or Hepalean or Panheprin).mp.

4. $\left(\mathrm{LMWH}^{\star}\right.$ or heparin ${ }^{\star}$ or nadroparin ${ }^{\star}$ or frixiparin ${ }^{\star}$ or enoxaparin ${ }^{\star}$ or clexane or klexane or lovenox or dalteparin or fragmin or ardeparin* or normiflo or tinzaparin or logiparin or innohep or certoparin or sandoparin or reviparin or clivarin ${ }^{\star}$ or danaproid or danaparoid or orgaran or antixarin or bemiparin* or hibor or zibor or ivor or badyket or semuloparin or parnaparin or tedelparin or fluxum or lohepa or lowhepa or parvoparin or seleparin* or tedelgliparin or lomoparan or orgaran or sulodexide or zivor or embolex or xaparin or clivarine or fondaparinux or Arixtra or UFH or Hepalean or Calcilean or Calciparine or Liquaemin or Liquemin or Multiparin or Novoheparin or Eparina or Hep-lock or Heparinate or Heparinic acid or Panheprin or Hepalean or Heparin Leo or Heparin Lock).mp.

\section{5. (FR-860 or FR 860 or FR860 or PK-10,169 or PK 10,169 or PK10,169 or PK-10169 or PK 10169 or PK10169 or EMT-967 or EMT 967 or EMT967 or EMT-966 or EMT 966 or EMT966 or CY 216 or CY-216 or CY216 or LMF CY-216 or LMF CY 216 or LMF CY216).mp.}

\section{6. exp coumarin derivative/}

7. (coumarin* or chromonar or coumestrol or esculin or isocoumarin* or psoralens or pyranocoumarins or umbelliferones).tw.

8. (4-Hydroxycoumarin* or warfarin* or acenocoumarol or nicoumalone or sinthrome or Sintrom or phenindione or dicoumarol or coumadin or phenprocoumon or phepromaron or ethyl-biscoumacetate or phenindione or Diphenadione or Tioclomarol or Racumi or Marcoumar or Marcumar or Falithrom or Jantoven or vitamin $\mathrm{K}$ antagonist ${ }^{\star}$ or VKA or fluindione or difenacoum or coumatetralyl or coumadin* or warfant or marevan or aldocumar).mp.

9. (Dermatan Sulfate or (Chondroitin Sulfate adj B) or Dermatan Sulfphate or DS 435 or MF-701 or OP-370 or b-Heparin or Mistral or Venorix).mp.

10. (thrombin adj inhibitor $\left.{ }^{\star}\right) \cdot \mathrm{mp}$.

11. (factor Xa inhibitor ${ }^{\star}$ or antithrombin* or anticoagul $\left.{ }^{\star}\right) . \mathrm{mp}$.

12. (rivaroxaban or Xarelto or apixaban or Eliquis or dabigatran etexilate or Edoxaban or Savaysa or Betrixaban or ximelagatran or pradaxa or lixiana or exanta or Darexaban or Otamixaban* or Razaxaban or Bivalirudin or Desirudin or Lepirudin or Melagatran or YM 150 or Iprivask or argatrovan or pradax* or Xarelto or BIBR-953 or BIBR-953ZW or BAY 59-7939 or BMS-562247 or DU-176 or DU-176b).mp.

\section{3. rivaroxaban/}




\section{4. dabigatran/}

15. (BIBR 953 or BIBR 953 ZW or Dabigatran or HSDB 8062 or Pradaxa or UNII-IOVM4M70GC).mp.

16. (target specific oral anticoagulant* or target-specific oral anticoagulant* or TSOAC* or new oral anticoagulant ${ }^{\star}$ or novel oral anticoagulant ${ }^{\star}$ or NOAC ${ }^{\star}$ or direct-acting oral anticoagulant ${ }^{\star}$ or direct acting oral anticoagulant ${ }^{\star}$ or direct oral anticoagulant ${ }^{\star}$ or DOAC*).ti,ab,kw.

\section{1 or 2 or 3 or 4 or 5 or 6 or 7 or 8 or 9 or 10 or 11 or 12 or 13 or 14 or 15 or 16}

\section{8. exp neoplasm/}

19. (malignan* or neoplasm* or cancer* or carcinoma* or adenocarcinoma* or tumour* or tumor ${ }^{\star}$ or glioma* or myeloma* or lymphoma* or leukemia* or leukaemia* or epithelioma* or adeno$\left.m a^{\star}\right)$.tw.

\section{18 or 19}

\subsection{7 and 20}

22. crossover procedure/

23. double-blind procedure/

24. randomised controlled trial/

25. single-blind procedure/

26. random*.mp.

27. factorial*.mp.

28. (crossover ${ }^{\star}$ or cross over ${ }^{\star}$ or cross-over $\left.^{\star}\right) \cdot \mathrm{mp}$.

29. placebo*.mp.

30. $\left(\right.$ double $^{\star}$ adj blind $\left.{ }^{\star}\right) \cdot m p$.

31. $\left(\right.$ singl ${ }^{\star}$ adj blind $\left.{ }^{\star}\right) \cdot m p$.

32. assign ${ }^{\star} \cdot \mathrm{mp}$.

33. allocat* $^{\star} \cdot \mathrm{mp}$.

34. volunteer ${ }^{\star} . \mathrm{mp}$.

\subsection{2 or 23 or 24 or 25 or 26 or 27 or 28 or 29 or 30 or 31 or 32 or 33 or 34}

\subsection{1 and 35}

\section{Systematic Review search strategy:}

1. exp anticoagulant agent/

2. (anticoagulant ${ }^{\star}$ or anti-coagulant $\left.{ }^{\star}\right)$.tw.

3. (Heparin or Adomiparin or alpha-Heparin or Arteven or "AVE-5026" or CY 222 or "Depo-Heparin" or "EINECS 232-681-7" or Fluxum or "Hed-heparin" or Hepathrom or HSDB 3094 or KB 101 or "Lipo-hepin" or M 118 or "M 118REH" or M118 or Octaparin or OP 386 or OP 622 or Pabyrin or Pularin or Subeparin or Sublingula or Thromboliquine or Triofiban or "UNII-1K5KDI46KZ" or "UNII-4QW4AN84NQ" or "UNII-5R0L1D739E" or "UNII-7UQ7X4Y489" or "UNII-9816XA9004" or "UNIIE47C0NF7LV" or "UNII-M316WT19D8" or "UNII-P776JQ4R2F" or "UNII-S79008V79F" or "UNIIT2410KM04A" or "UNII-V72OT3K19I" or "UNII-VL0L558GCB" or Vetren or Vitrum AB or enoxaparin* or klexane or lovenox or fragmin* or normiflo or logiparin or innohep or danaproid or danaparoid or orgaran or antixarin or hibor or zibor or ivor or badyket or lohepa or lowhepa or seleparin* or tedelgliparin or lomoparan or orgaran or sulodexide or zivor or embolex or xaparin or fondaparinux 
or Arixtra or UFH or Hepalean or Calcilean or Calciparine or "Hep-lock" or enoxaparin* or klexane or lovenox or fragmin* or normiflo or logiparin or innohep or danaproid or danaparoid or orgaran or antixarin or hibor or zibor or ivor or badyket or lohepa or lowhepa or seleparin* or tedelgliparin or lomoparan or orgaran or sulodexide or zivor or embolex or xaparin or fondaparinux or Arixtra or UFH or Hepalean or Panheprin).mp.

4. $\left(\mathrm{LMWH}^{\star}\right.$ or heparin ${ }^{\star}$ or nadroparin ${ }^{\star}$ or frixiparin* or enoxaparin* or clexane or klexane or lovenox or dalteparin or fragmin or ardeparin* or normiflo or tinzaparin or logiparin or innohep or certoparin or sandoparin or reviparin or clivarin* or danaproid or danaparoid or orgaran or antixarin or bemiparin* or hibor or zibor or ivor or badyket or semuloparin or parnaparin or tedelparin or fluxum or lohepa or lowhepa or parvoparin or seleparin* or tedelgliparin or lomoparan or orgaran or sulodexide or zivor or embolex or xaparin or clivarine or fondaparinux or Arixtra or UFH or Hepalean or Calcilean or Calciparine or Liquaemin or Liquemin or Multiparin or Novoheparin or Eparina or Hep-lock or Heparinate or Heparinic acid or Panheprin or Hepalean or Heparin Leo or Heparin Lock).mp.

5. (FR-860 or FR 860 or FR860 or PK-10,169 or PK 10,169 or PK10,169 or PK-10169 or PK 10169 or PK10169 or EMT-967 or EMT 967 or EMT967 or EMT-966 or EMT 966 or EMT966 or CY 216 or CY-216 or CY216 or LMF CY-216 or LMF CY 216 or LMF CY216).mp.

6. exp coumarin derivative/

7. (coumarin* or chromonar or coumestrol or esculin or isocoumarin* or psoralens or pyranocoumarins or umbelliferones).tw.

8. (4-Hydroxycoumarin* or warfarin* or acenocoumarol or nicoumalone or sinthrome or Sintrom or phenindione or dicoumarol or coumadin or phenprocoumon or phepromaron or ethyl-biscoumacetate or phenindione or Diphenadione or Tioclomarol or Racumi or Marcoumar or Marcumar or Falithrom or Jantoven or vitamin $\mathrm{K}$ antagonist ${ }^{\star}$ or VKA or fluindione or difenacoum or coumatetralyl or coumadin* or warfant or marevan or aldocumar).mp.

9. (Dermatan Sulfate or (Chondroitin Sulfate adj B) or Dermatan Sulfphate or DS 435 or MF-701 or OP-370 or b-Heparin or Mistral or Venorix).mp.

10. (thrombin adj inhibitor $\left.{ }^{\star}\right) \cdot \mathrm{mp}$.

11. (factor Xa inhibitor ${ }^{\star}$ or antithrombin ${ }^{\star}$ or anticoagul $\left.{ }^{\star}\right)$.mp.

12. (rivaroxaban or Xarelto or apixaban or Eliquis or dabigatran etexilate or Edoxaban or Savaysa or Betrixaban or ximelagatran or pradaxa or lixiana or exanta or Darexaban or Otamixaban* or Razaxaban or Bivalirudin or Desirudin or Lepirudin or Melagatran or YM 150 or Iprivask or argatrovan or pradax ${ }^{\star}$ or Xarelto or BIBR-953 or BIBR-953ZW or BAY 59-7939 or BMS-562247 or DU-176 or DU-176b).mp.

13. rivaroxaban/

14. dabigatran/

15. (BIBR 953 or BIBR 953 ZW or Dabigatran or HSDB 8062 or Pradaxa or UNII-IOVM4M70GC).mp.

16. (target specific oral anticoagulant* or target-specific oral anticoagulant ${ }^{\star}$ or TSOAC ${ }^{\star}$ or new oral anticoagulant ${ }^{\star}$ or novel oral anticoagulant ${ }^{\star}$ or NOAC ${ }^{\star}$ or direct-acting oral anticoagulant ${ }^{\star}$ or direct acting oral anticoagulant* ${ }^{\star}$ or direct oral anticoagulant ${ }^{\star}$ or DOAC*).ti,ab,kw.

\section{1 or 2 or 3 or 4 or 5 or 6 or 7 or 8 or 9 or 10 or 11 or 12 or 13 or 14 or 15 or 16}

18. exp neoplasm/

19. (malignan* or neoplasm* or cancer ${ }^{\star}$ or carcinoma* or adenocarcinoma* or tumour* or tumor ${ }^{\star}$ or glioma* or myeloma* or lymphoma* or leukemia* or leukaemia* or epithelioma* or adenoma*).tw.

\section{18 or 19}

21. 17 and 20

Anticoagulation for the long-term treatment of venous thromboembolism in people with cancer (Review) Copyright $\odot 2023$ The Cochrane Collaboration. Published by John Wiley \& Sons, Ltd. 


\section{2. exp Meta Analysis/}

23. ((meta adj analy\$) or metaanalys\$).tw.

24. (systematic adj (review\$1 or overview\$1)).tw.

25. 22 or 23 or 24

26. cancerlit.ab.

27. cochrane.ab.

28. embase.ab.

29. (psychinfo or psycinfo).ab.

30. (cinahl or cinhal).ab.

31. science citation index.ab.

32. bids.ab.

33. 26 or 27 or 28 or 29 or 30 or 31 or 32

34. reference lists.ab.

35. bibliograph\$.ab.

36. hand-search\$.ab.

37. manual search\$.ab.

38. relevant journals.ab.

39. 34 or 35 or 36 or 37 or 38

40. data extraction.ab.

41. selection criteria.ab.

42. 40 or 41

43. review.pt.

44. 42 and 43

45. letter.pt.

46. editorial.pt.

47. animal/

48. human/

49. 47 not (47 and 48)

50.45 or 46 or 49

51.25 or 33 or 39 or 44

52. 51 not 50

53. 21 and 52 
\#2 (LMWH* or heparin* or nadroparin* or frixiparin* or enoxaparin* or clexane or klexane or lovenox or dalteparin or fragmin or ardeparin* or normiflo or tinzaparin or logiparin or innohep or certoparin or sandoparin or reviparin or clivarin* or danaproid or danaparoid or orgaran or antixarin or bemiparin* or hibor or zibor or ivor or badyket or semuloparin or parnaparin or tedelparin or fluxum or lohepa or lowhepa or parvoparin or seleparin* or tedelgliparin or lomoparan or orgaran or sulodexide or zivor or embolex or xaparin or clivarine or fondaparinux or Arixtra or UFH or Hepalean or Calcilean or Calciparine or Liquaemin or Liquemin or Multiparin or Novoheparin or Eparina or Hep-lock or Heparinate or Heparinic acid or Panheprin or Hepalean or Heparin Leo or Heparin Lock)

\#3 FR-860 or FR 860 or FR860 or PK-10,169 or PK 10,169 or PK10,169 or PK-10169 or PK 10169 or PK10169 or EMT-967 or EMT 967 or EMT967 or EMT-966 or EMT 966 or EMT966 or CY 216 or CY-216 or CY 216 or LMF CY-216 or LMF CY 216 or LMF CY216

\#4 MeSH descriptor: [Coumarins] explode all trees

\#5 (4-Hydroxycoumarin* or warfarin* or acenocoumarol or nicoumalone or sinthrome or Sintrom or phenindione or dicoumarol or coumadin or phenprocoumon or phepromaron or ethyl-biscoumacetate or phenindione or Diphenadione or Tioclomarol or Racumi or Marcoumar or Marcumar or Falithrom or Jantoven or vitamin K antagonist ${ }^{\star}$ or VKA or fluindione or difenacoum or coumatetralyl)

\#6 (Dermatan Sulfate or (Chondroitin Sulfate adj B) or Dermatan Sulfphate or DS 435 or MF-701 or OP-370 or b-Heparin or Mistral or Venorix)

\#7 thrombin near inhibitor ${ }^{\star}$

\#8 factor Xa inhibitor ${ }^{\star}$ or antithrombin* or anticoagul ${ }^{*}$

\#9 (rivaroxaban or Xarelto or apixaban or Eliquis or dabigatran etexilate or Edoxaban or Savaysa or Betrixaban or ximelagatran or pradaxa or lixiana or exanta or Darexaban or Otamixaban* or Razaxaban or Bivalirudin or Desirudin or Lepirudin or Melagatran or YM 150 or Iprivask or argatrovan or pradax* or Xarelto or BIBR-953 or BIBR-953ZW or BAY 59-7939 or BMS-562247 or DU-176 or DU-176b).mp.

\#10 MeSH descriptor: [Rivaroxaban] this term only

\#11 MeSH descriptor: [Dabigatran] this term only

\#12 target specific oral anticoagulant* or target-specific oral anticoagulant* or TSOAC* or new oral anticoagulant ${ }^{\star}$ or novel oral anticoagulant ${ }^{\star}$ or NOAC* or direct-acting oral anticoagulant* or direct acting oral anticoagulant ${ }^{\star}$ or direct oral anticoagulant ${ }^{\star}$ or DOAC ${ }^{\star}$

$\# 13 \# 1$ or \#2 or \#3 or \#4 or \#5 or \#6 or \#7 or \#8 or \#9 or \#10 or \#11 or \#12

\#14 MeSH descriptor: [Neoplasms] explode all trees

\#15 malignan* or neoplasm* or cancer* or carcinoma* or adenocarcinoma* or tumour* or tumor* or glioma* or myeloma* or lymphoma* or leukemia* or leukaemia* or epithelioma* or adenoma*

\#16 \#14 or \#15

\#17 \#13 and \#16

\section{Appendix 3. Detailed results of sensitivity analyses}

\begin{tabular}{ll}
\hline Comparison & LMWH vs VKA \\
\hline
\end{tabular}


(Continued)

\section{CCA effect estimate}

Sensitivity analysis

\begin{tabular}{ll}
\hline RI $\mathbf{1 . 5}$ intervention $\mathbf{1}$ control & RR 0.61 (95\% Cl 0.45 to 0.82) \\
\hline RI $\mathbf{2}$ intervention $\mathbf{1}$ control & RR 0.62 (95\% Cl 0.46 to 0.83) \\
\hline RI $\mathbf{3}$ intervention $\mathbf{1}$ control & RR $0.64(95 \% \mathrm{Cl} 0.48$ to 0.86$)$ \\
\hline RI $\mathbf{5}$ intervention $\mathbf{1}$ control & RR $0.68(95 \% \mathrm{Cl} 0.50$ to 0.91$)$ \\
\hline
\end{tabular}

\section{Comparison}

\section{Outcome}

\section{CCA effect estimate}

Sensitivity analysis

\begin{tabular}{ll}
\hline RI $\mathbf{1 . 5}$ intervention ${ }^{1}$ control & RR 0.65 (95\% Cl 0.48 to 0.89) \\
\hline RI $\mathbf{2}$ intervention ${ }^{1}$ control & RR 0.67 (95\% Cl 0.50 to 0.90) \\
\hline RI $\mathbf{3}$ intervention ${ }^{1}$ control & RR 0.71 (95\% Cl 0.54 to 0.93) \\
\hline RI $\mathbf{5}$ intervention $\mathbf{1}$ control & RR 0.75 (95\% Cl 0.57 to 0.99)
\end{tabular}

\begin{tabular}{|c|c|}
\hline Comparison & DOAC vs LMWH \\
\hline Outcome & Minor bleeding \\
\hline CCA effect estimate & RR $1.58(95 \% \mathrm{Cl} 1.15$ to 2.16$)$ \\
\hline \multicolumn{2}{|l|}{ Sensitivity analysis } \\
\hline RI 1.5 intervention 1 control & RR $1.53(95 \% \mathrm{Cl} 1.11$ to 2.11$)$ \\
\hline RI 2 intervention 1 control & RR 1.48 (95\% Cl 1.07 to 2.07$)$ \\
\hline RI 3 intervention $\mathbf{1}$ control & RR 1.41 (95\% $\mathrm{Cl} 0.99$ to 2.00$)$ \\
\hline RI 5 intervention $\mathbf{1}$ control & RR 1.28 (95\% Cl 0.87 to 1.88$)$ \\
\hline
\end{tabular}

\section{DOAC vs LMWH}

Recurrent VTE

RR 0.63 ( $95 \%$ Cl 0.45 to 0.88 )

$.63(95 \% \mathrm{Cl} 0.45$ to 0.88$)$


WHAT'S NEW

\begin{tabular}{lll}
\hline Date & Event & Description \\
\hline 21 December 2022 & Amended & $\begin{array}{l}\text { This is a Living Systematic Review. Searches are run and } \\
\text { screened monthly. Last search date 14 December 2022 (no new } \\
\text { studies found). As such, results of all included studies identified } \\
\text { have been incorporated. The conclusions of this Cochrane Re- } \\
\text { view are therefore considered up to date. [Enter text here] }\end{array}$ \\
\hline
\end{tabular}

\section{HISTOR Y}

Protocol first published: Issue 3, 2007

Review first published: Issue 2, 2008

\begin{tabular}{lll}
\hline Date & Event & Description \\
\hline 26 October 2022 & Amended & $\begin{array}{l}\text { This is a Living Systematic Review. Searches are run and } \\
\text { screened monthly. Last search date 14 October 2022 (no new } \\
\text { studies found). As such, results of all included studies identified } \\
\text { have been incorporated. The conclusions of this Cochrane Re- } \\
\text { view are therefore considered up to date. }\end{array}$ \\
\hline
\end{tabular}

13 June $2022 \quad$ Amended

This is a Living Systematic Review. Searches are run and screened monthly. Last search date 14 May 2022 (no new studies found). As such, results of all included studies identified have been incorporated. The conclusions of this Cochrane Review are therefore considered up to date.

29 December $2021 \quad$ Amended

This is a Living Systematic Review. Searches are run and screened monthly. Last search date 14 December 2021 (no new studies found). As such, results of all included studies identified have been incorporated. The conclusions of this Cochrane Review are therefore considered up to date.

14 April $2021 \quad$ Amended

This is a Living Systematic Review. Searches are run and screened monthly. Last search date 14 May 2021. We have identified and incorporated four new studies (Agnelli 2020 (Caravaggio); El Mokadem 2020; McBane 2019 (ADAM-VTE); Young 2018 (SELECT-D)). Conclusion has changed accordingly. xxxx The conclusions of this Cochrane Review are therefore considered up to date.

29 October $2020 \quad$ Amended

This is a Living Systematic Review. Searches are run and screened monthly. Last search date 14 October 2020. We have identified the full-text of a previously identified abstract. As such, results of all available included studies identified have been incorporated. The conclusions of this Cochrane Review are therefore considered up to date.

17 June $2020 \quad$ Amended

This is a Living Systematic Review. Searches are run and screened monthly. Last search date 14 May 2020. We have identified the full-text of a previously identified abstract. As such, results of all available included studies identified have been incor- 


\begin{tabular}{|c|c|c|}
\hline Date & Event & Description \\
\hline & & $\begin{array}{l}\text { porated. The conclusions of this Cochrane Review are therefore } \\
\text { considered up to date. }\end{array}$ \\
\hline 12 March 2020 & Amended & $\begin{array}{l}\text { This is a Living Systematic Review. Searches are run and } \\
\text { screened monthly. Last search date } 14 \text { February } 2020 \text {. We have } \\
\text { identified the full-text of a previously identified abstract. As such, } \\
\text { results of all available included studies identified have been in- } \\
\text { corporated. The conclusions of this Cochrane Review are there- } \\
\text { fore considered up to date. }\end{array}$ \\
\hline
\end{tabular}

2 January $2020 \quad$ Amended

This is a Living Systematic Review. Searches are run and screened monthly. Last search date 14 November 2019. We have identified the full-text of a previously identified abstract. As such, results of all available included studies identified have been incorporated. The conclusions of this Cochrane Review are therefore considered up to date.

7 October $2019 \quad$ Amended

This is a Living Systematic Review. Searches are run and screened monthly. Last search date 14 August 2019. We have identified the full-text of a previously identified abstract. As such, results of all available included studies identified have been incorporated. The conclusions of this Cochrane Review are therefore considered up to date.

\begin{tabular}{lll}
\hline 25 July 2019 & Amended & Typographical error corrected. \\
\hline 9 July 2019 & Amended & $\begin{array}{l}\text { This is a Living Systematic Review. Searches are run and } \\
\text { screened monthly. Last search date 14 June 2019. We have iden- } \\
\text { tified the full-text of a previously identified abstract. As such, re- } \\
\text { sults of all available included studies identified have been incor- } \\
\text { porated. The conclusions of this Cochrane Review are therefore } \\
\text { considered up to date. }\end{array}$
\end{tabular}

9 May $2019 \quad$ Amended

This is a Living Systematic Review. Searches are run and screened monthly. Last search date 24 April 2019. We have identified the full-text of a previously identified abstract. As such, results of all available included studies identified have been incorporated. The conclusions of this Cochrane Review are therefore considered up to date.

25 February $2019 \quad$ Amended

This is a Living Systematic Review. Searches are run and screened monthly. Last search date 14 February 2019 when we identified the full-text of a previously identified abstract. As such, results of all available included studies identified have been incorporated. The conclusions of this Cochrane Review are therefore considered up to date.

29 November $2018 \quad$ Amended

This is a Living Systematic Review. Searches are run and screened monthly. Last search date 14 November 2018 (no new studies found). As such, results of all included studies identified have been incorporated. The conclusions of this Cochrane Review are therefore considered up to date.

1 October $2018 \quad$ Amended

This is a Living Systematic Review. Searches are run and screened monthly. Last search date 14 September 2018 (no new studies found). As such, results of all included studies identified have been incorporated. The conclusions of this Cochrane Review are therefore considered up to date. 


\begin{tabular}{lll}
\hline Date & Event & Description \\
\hline 9 August 2018 & Amended & $\begin{array}{l}\text { This is a Living Systematic Review. Searches are run and } \\
\text { screened monthly. Last search date 14 July 2018 (no new stud- } \\
\text { ies found). As such, results of all included studies identified have } \\
\text { been incorporated. The conclusions of this Cochrane Review are } \\
\text { therefore considered up to date. }\end{array}$
\end{tabular}

28 June $2018 \quad$ Amended

This is a Living Systematic Review. Searches are run and screened monthly. Last search date 14 May 2018.

New comparison added (direct oral anticoagulant (DOAC) versus low molecular weight heparin (LMWH)). Two new studies found for the comparison DOAC versus LMWH (one published as full text and the other as an abstract). As such, results of all included studies identified were incorporated. The conclusions of this Cochrane Review are therefore considered up to date.

\begin{tabular}{lll}
\hline 28 June 2018 & Amended & Declaration of interest updated. \\
\hline 14 May 2018 & $\begin{array}{l}\text { New citation required but conclusions } \\
\text { have not changed }\end{array}$ & Updated author list. \\
\hline 14 May 2018 & New search has been performed & $\begin{array}{l}\text { This is a Living Systematic Review. Searches are run and } \\
\text { screened monthly. Last search date 14 May 2018. }\end{array}$ \\
& $\begin{array}{l}\text { New comparison added (direct oral anticoagulant (DOAC) versus } \\
\text { low molecular weight heparin (LMWH)). Two new studies found } \\
\text { for the comparison DOAC versus LMWH (one published as full } \\
\text { text and the other as an abstract). As such, results of all includ- } \\
\text { ed studies identified were incorporated. The conclusions of this } \\
\text { Cochrane Review are therefore considered up to date. }\end{array}$ \\
\hline
\end{tabular}

\begin{tabular}{lll}
\hline 25 June 2014 & Amended & Table format update \\
\hline 4 June 2014 & $\begin{array}{l}\text { New citation required but conclusions } \\
\text { have not changed }\end{array}$ & $\begin{array}{l}\text { Data abstraction verified and detailed statistical data included } \\
\text { as appendix } \\
\text { Data reanalyzed by using a complete case analysis approach for } \\
\text { the primary meta-analysis }\end{array}$ \\
\hline 9 February 2013 & New search has been performed & Search Updated \\
\hline 28 November 2012 & Amended & Author contact details amended \\
\hline 9 May 2011 & $\begin{array}{l}\text { New citation required but conclusions } \\
\text { have not changed }\end{array}$ & $\begin{array}{l}\text { One new randomized controlled trial (RCT) identified and added } \\
\text { to review. New authors also added. }\end{array}$ \\
\hline 9 May 2011 & New search has been performed & Search updated 7 February 2010. One new RCT was identified. \\
\hline
\end{tabular}

\section{CONTRIBUTIONS OF AUTHORS}

LAK: searching, screening, full-text retrieval, data extraction, data analysis, data interpretation, manuscript drafting, review co-ordination. $\mathrm{MBH}$ : screening, full-text retrieval, data extraction, manuscript drafting.

IGT: screening, data extraction, manuscript drafting.

CFM: screening.

FA: data extraction.

IT: screening, data extraction. 
FS: screening.

$M B$ : screening.

VEDY: screening.

HJS: protocol development, data interpretation, methodological expertise.

EAA: protocol development, data analysis, data interpretation, manuscript drafting, methodological expertise, review co-ordination.

\section{DECLARATIONS OF INTEREST}

LAK: no conflicts of interest $\mathrm{MBH}$ : no conflicts of interests IGT: no conflicts of interests

CFM: no conflicts of interests

IT: no conflicts of interests

FS: no conflicts of interests.

MB: no conflicts of interests

VEDY: no conflicts of interests

HJS: panel member of the ASH VTE in Cancer patients, Vice-Chair of the ASH VTE guidelines and played various leadership roles from 1999 until 2014 with ACCP VTE guidelines.

EAA: served on the executive committee the ACCP Antithrombotic Therapy Guidelines published in 2016

\section{SOURCES OF SUPPORT}

\section{Internal sources}

- No sources of support provided

\section{External sources}

- National Institute for Health Research Cochrane Review Incentive Scheme 2016. Award reference Number 16/72/24, UK

This project was supported by the National Institute for Health Research (NIHR), via Cochrane Infrastructure funding to the Cochrane Gynaecological, Neuro-oncology and Orphan Cancer Group

- American Society of Hematology, USA

This project was supported by the American Society of Hematology

\section{DIFFERENCES BETWEEN PROTOCOL AND REVIEW}

- We previously reported using the 'related citation' feature in PubMed and 'citation tracking' of included studies in Web of Science Core Collection to identify additional articles. We stopped applying this method since February 2018, as over two years (February 2016 till February 2018), this method did not retrieve any additional reference compared to the findings of the database automated search.

- We have updated our search strategy (Appendix 2).

- For feasibility reasons, we will be updating the status of this systematic review every six months instead of every month.

- We focused in the primary analysis on participants with active cancer.

- We stratified major and minor bleeding for the comparison DOAC versus LMWH as follows:

- Gastrolntestinal (GI) tract bleeding

- Upper GI bleeding

- Lower GI bleeding

- non-Gl bleeding

\section{N DEX TERMS}

\section{Medical Subject Headings (MeSH)}

Administration, Oral; Anticoagulants [adverse effects] [^therapeutic use]; Azetidines [therapeutic use]; Benzimidazoles [therapeutic use]; Benzylamines [therapeutic use]; beta-Alanine [analogs \& derivatives] [therapeutic use]; Dabigatran [therapeutic use]; Hemorrhage [chemically induced]; Heparin, Low-Molecular-Weight [therapeutic use]; Neoplasms [ ${ }^{\star}$ complications]; Oligosaccharides [therapeutic use]; Randomized Controlled Trials as Topic; Venous Thromboembolism [ ${ }^{\star}$ drug therapy] [etiology] [mortality]; Vitamin K [antagonists \& inhibitors] 


\section{MeSH check words}

Humans 